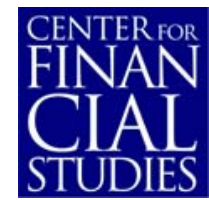

No. $2007 / 23$

International Investment Positions and Exchange Rate Dynamics:

A Dynamic Panel Analysis

Michael Binder and Christian Offermanns 


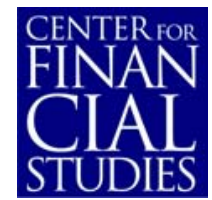

\section{Center for Financial Studies}

The Center for Financial Studies is a nonprofit research organization, supported by an association of more than 120 banks, insurance companies, industrial corporations and public institutions. Established in 1968 and closely affiliated with the University of Frankfurt, it provides a strong link between the financial community and academia.

The CFS Working Paper Series presents the result of scientific research on selected topics in the field of money, banking and finance. The authors were either participants in the Center's Research Fellow Program or members of one of the Center's Research Projects.

If you would like to know more about the Center for Financial Studies, please let us know of your interest.

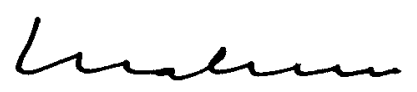

Prof. Dr. Jan Pieter Krahnen

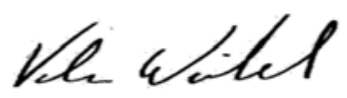

Prof. Volker Wieland, Ph.D. 


\title{
International Investment Positions and Exchange Rate Dynamics: A Dynamic Panel Analysis*
}

\author{
Michael Binder ${ }^{1}$ and Christian Offermanns ${ }^{2}$
}

\author{
August 2007
}

\begin{abstract}
:
In this paper we revisit medium- to long-run exchange rate determination, focusing on the role of international investment positions. To do so, we develop a new econometric framework accounting for conditional long-run homogeneity in heterogeneous dynamic panel data models. In particular, in our model the long-run relationship between effective exchange rates and domestic as well as weighted foreign prices is a homogeneous function of a country's international investment position. We find rather strong support for purchasing power parity in environments of limited negative net foreign asset to GDP positions, but not outside such environments. We thus argue that the purchasing power parity hypothesis holds conditionally, but not unconditionally, and that international investment positions are an essential component to characterizing this conditionality. Finally, we adduce evidence that whether deterioration of a country's net foreign asset to GDP position leads to a depreciation of that country's effective exchange rate depends on its rate of inflation relative to the rate of inflation abroad as well as its exposure to global shocks.
\end{abstract}

JEL Classification: F31, F37, C23

Keywords: Exchange Rate Determination, International Financial Integration, Dynamic Panel Data Models

\footnotetext{
* We are grateful for comments and suggestions from Heinz Herrmann, Vanessa Smith and J“urgen von Hagen, as well as from seminar and conference participants at the Deutsche Bundesbank, the Bank of England, University of Bonn, European University Institute, Goethe University Frankfurt and Makro“okonomischer Ausschuss des Vereins f"ur Socialpolitik. Of course, all remaining errors are our own. The views expressed in this paper are those of the authors and do not necessarily reflect the opinion of the Deutsche Bundesbank.

1 Frankfurt University and Center for Financial Studies; email: mbinder@wiwi.uni-frankfurt.de

2 Frankfurt University and Deutsche Bundesbank; email: offerman@wiwi.uni-frankfurt.de
} 


\section{Introduction}

Research on exchange rate dynamics constitutes a continued cornerstone of applied economic investigations. A sizeable fraction of these investigations have aimed at understanding the driving forces of medium- to long-run exchange rate dynamics. Nevertheless, little consensus has been reached. In particular, in the quest to characterize medium- to long-run anchors for the fluctuations of exchange rates, the purchasing power parity (PPP) hypothesis has received support by some studies, yet has been rejected by others. While these differences in empirical findings may in part be attributed to choice of econometric methodology, the differences have also emerged due to different currency pairs and/or different time periods being considered.

For research in this area to move forward, it thus appears essential to view the PPP hypothesis as (at most) conditionally valid and to pay close consideration to the interaction between exchange rate fluctuations on the one hand and the macroeconomic as well as financial environment within which the pricing of currencies occurs on the other hand. Arguably, one of the most striking changes in this environment over the last few decades has been the growth of cross-country capital flows and the cumulative international investment positions they imply. As argued for example by Lane and Milesi-Ferretti (2005), "financial globalization [has been] one of the key trends that has reshaped the global economy".

In this paper, we study the interaction between medium- to long-run exchange rate dynamics and international investment positions in equity, foreign direct investment and debt. We analyze to what extent the PPP hypothesis may be viewed as an anchor for the pricing of a currency over medium- to long-run horizons if conditioned on the international investment position of the country issuing the currency.

Previous work on the PPP hypothesis (for example, Taylor, Peel and Sarno, 2001, and Binder, Pesaran and Sharma, 2004) has argued that mean reversion of real exchange rates only occurs under sufficiently large imbalances and/or arbitrage opportunities for foreign exchange market participants. Here, we relate these bands of real exchange rate reversion to a country's international investment position: We conjecture that if foreign exchange market participants perceive this investment position to be sufficiently imbalanced to require correction, they expect a return to macroeconomic fundamentals that includes correction of the exchange rate towards a plausible anchor, possibly the level of the exchange rate predicted by PPP. Such a correction may help to adjust the international investment position both through current account and valuation effects and in any case may signal the markets' expectation of a reversion towards medium- to long-run fundamentals. We also conjecture that under very severe imbalances in a country's international investment position foreign exchange market participants may lose confidence in the relevance of macroeconomic funda- 
mentals for the pricing of the country's currency, and therefore again, as in the absence of sufficiently large imbalances, will pay little - if any - attention to PPP.

We test these hypotheses in this paper and more generally provide a characterization of the role of international investment positions for medium- to long-run exchange rate dynamics using a panel of 71 countries over the time period 1970 to 2004 . We propose and implement a new dynamic panel data model for our analysis. Our panel model has a variety of appealing features: In line with existing state of the art cross-country panel models in the literature, our model explicitly distinguishes between short- and long-run dynamics, does not impose untenable exogeneity restrictions, is valid in the presence of unit roots in the series being considered, and allows for heterogeneous short-run dynamics of these series across countries. It moves beyond the models presently available in the literature by introducing conditional homogeneity across countries in the long-run relation between the series. We model the conditional long-run homogeneity both parametrically using flexible functional form polynomials (resulting in what we call the conditional pooled mean group (CPMG) panel model) and non-parametrically using local kernels (resulting in what we call the state kernel mean group (SKMG) panel model). This econometric framework is applicable - and perhaps appealing - for a wide range of panel data sets with sufficiently large time dimension for which traditional pooling restrictions are not tenable.

Our main empirical results are as follows: We find rather strong support for the PPP hypothesis in environments of limited negative international investment positions as measured by the net foreign asset (NFA) to GDP ratio. In such environments the coefficients in the long-run relation between effective nominal exchange rates, domestic prices and weighted foreign prices are (economically) close to their predicted values under PPP. Furthermore, the speed of adjustment towards the long-run relation is, in light of the estimates typically obtained in the previous literature, surprisingly fast, at less than two years half-life of shocks to the PPP relation. We also document that in environments of large negative, zero or positive NFA to GDP positions the PPP hypothesis does not provide a relevant medium- to long-run anchor for the pricing of currencies. Our robustness analysis finds that qualitatively our results are unlikely to be driven by features of the macroeconomic and financial environment other than the international investment position of a country. We document that there is no sensitivity of the range of NFA to GDP positions for which the PPP hypothesis applies to a country's exchange rate regime. While the range of NFA to GDP positions for which the PPP hypothesis applies does feature some sensitivity to a country's income level and degree of price variability, even when taking into account these features of a country's macroeconomic environment, the conditioning of PPP on a country's international investment position remains important. We finally adduce evidence that how deterioration of a country's NFA to GDP position affects changes in that country's effective exchange rate 
depends on its rate of inflation relative to the rate of inflation abroad as well as its exposure to global shocks.

The paper is organized as follows: In Section 2 we discuss the relation of our work to previous literature, both that on medium- to long-run exchange rate dynamics and that on cross-country panel models. Section 3 develops the CPMG and SKMG panel models. We outline the main features of our newly assembled database on international capital flows and international investment positions in Section 4 of the paper. Our empirical findings are presented in Section 5, and Section 6 concludes and discusses directions for future research. Five appendices provide details on the assembly of our database and on various aspects of the implementation of the CPMG and SKMG models.

\section{Relation to the Literature}

\subsection{Exchange Rate Dynamics}

While there is an enormous body of literature investigating the validity of the purchasing power parity hypothesis, ${ }^{1}$ rather limited attention has been paid to investigating the interaction between exchange rate fluctuations and the macroeconomic as well as financial environment within which the pricing of currencies occurs. Two of the exceptions are Taylor, Peel and Sarno (2001) and Binder, Pesaran and Sharma (2004). The former proposed a nonlinear model for medium- to long-run real exchange rate dynamics, capturing that mean reversion of real exchange rates would only occur if these deviated sufficiently strongly from the PPP anchor. ${ }^{2}$ The latter - using dynamic panel models subject to simple sample splits argued that the empirical validity of the PPP hypothesis is linked to the volatility of domestic prices, and that below a minimum threshold of price volatility arbitrage opportunities would be too small for PPP to hold.

Neither of these two papers considered the link between exchange rate determination and a country's international investment position. A theoretical basis for this link was established by Cavallo and Ghironi (2002) who build on the model by Obstfeld and Rogoff (1995) and make the case for a dependence of exchange rates on net foreign assets both under a model with flexible and with sticky prices. Important papers investigating this link empirically include Lane and Milesi-Ferretti (2004) and Cheung, Chinn and Pascual (2005). Both of these papers consider a linear regression specification with the real exchange rate as the dependent variable and a measure of the NFA position as one of the regressors. Our approach

\footnotetext{
${ }^{1}$ For a recent review of the PPP literature see, for example, Taylor and Taylor (2004). Engel, Mark and West (2007) discuss state of the art exchange rate modelling beyond the PPP literature also.

${ }^{2}$ Baum, Barkoulas and Caglayan (2001) argue in a similar way in a model separating between nominal exchange rates and domestic as well as foreign prices.
} 
will not be to add the NFA position as an additional regressor, implying unconditional rejection of the PPP hypothesis if this regressor is significant and unconditional support for the PPP hypothesis if this regressor is insignificant, but to allow for the possibility that PPP is conditionally valid for certain NFA positions. We think that our dynamic model with conditionally homogeneous long-run relations is a more informative means to characterize the link between a country's international investment position and its medium- to long-run exchange rate dynamics than the default linear regression approach of tacking on the international investment position as an additional regressor - for the reasons that our model allows for bands of real exchange rate reversion (as well as lack thereof), is able to characterize the economic determinants of these bands and does not impose a monotonic relationship between changes in a country's international investment position and its exchange rate adjustment.

\subsection{Panel Data and Varying Parameter Models}

Key to the understanding of the recent econometric literature on cross-country dynamic panel data models is the result by Pesaran and Smith (1995) that if a model's slope coefficients vary across countries, whether randomly or systematically, then the means of the coefficients cannot be estimated consistently using a model imposing cross-country homogeneity of the slope coefficients (and only allowing for structural heterogeneity in the form of random or fixed effects). To obtain consistent estimators of the means of the slope coefficients, Pesaran and Smith (1995) proposed the mean group (MG) estimator based on the idea of averaging the estimates obtained from country-specific time-series regressions. This MG estimator has the drawback of not allowing for the efficiency gains that are feasible when some economic features are common across countries. While short-run dynamics beyond some common shocks are rather unlikely to share common features across a broad range of countries, common features often are likely to be present in long-run relationships. This insight is exploited by the pooled mean group (PMG) estimator of Pesaran, Shin and Smith (1999), which imposes homogeneity of the slope coefficients entering the long-run relationships, but allows for unrestricted heterogeneity of the coefficients characterizing the short-run dynamics.

The dynamic panel model we propose in this paper addresses situations where the homogeneity of the slope coefficients entering the long-run relationships does not hold unconditionally, but rather is tied to certain features of the macroeconomic and financial environment. In such settings, the PMG estimator would yield inconsistent estimates of the long-run slope coefficients, while the MG estimator would still suffer from lack of efficiency. We will pursue two approaches to modelling the dependence of the long-run slope coefficients on features of the macroeconomic and financial environment. Our first approach is parametric, modelling the state dependence using flexible functional form polynomials. Our second approach involves modelling the state dependence via non-parametric kernel methods. The statistical 
literature on non-parametric varying parameter models in static regression settings on which our modelling approach builds is quite extensive, see for example Fan and Zhang (1999). Kumar and Ullah (2000) have employed a related non-parametric approach in the context of a univariate dynamic panel model studying convergence of cross-country output growth.

\section{Econometric Methodology}

\subsection{The Mean Group and Pooled Mean Group Panel Models}

We begin by reviewing the dynamic panel models, mean group (MG) and pooled mean group (PMG), on which our proposed new model does build. Let us consider the following panel version of an autoregressive distributed lag, $\operatorname{ARDL}(p, q)$, model:

$$
y_{i t}=\omega_{i}+\sum_{k=1}^{p} \rho_{i k} y_{i, t-k}+\sum_{k=0}^{q} \boldsymbol{\varrho}_{i k}^{\prime} \boldsymbol{x}_{i, t-k}+u_{i t},
$$

where $i=1,2, \ldots, N$ indexes countries, $t=1,2, \ldots, T_{i}$ indexes time periods, $y_{i t}$ denotes the dependent variable (with coefficients $\rho_{i k}$ on its lagged values), $\omega_{i}$ represents the countryspecific intercept term (fixed effect), and $\boldsymbol{x}_{i t}$ and $\boldsymbol{\varrho}_{i k}$ represent $(m \times 1)$ vectors of explanatory variables and coefficients, respectively. ${ }^{3}$ We assume that $\min _{i}\left(T_{i}\right)$ is sufficiently large so that the ARDL model in (1) can be estimated for each country separately.

To allow for cross-sectional correlation of the error terms, we specify $u_{i t}$ as:

$$
u_{i t}=\boldsymbol{\lambda}_{i}^{\prime} \boldsymbol{f}_{t}+\varepsilon_{i t}
$$

such that the source of error term dependencies across countries is captured by the common factors $\boldsymbol{f}_{t}$, whereas the impacts of these factors on each country are governed by the idiosyncratic loadings in $\boldsymbol{\lambda}_{i}$. The error component $\varepsilon_{i t}$ is assumed to be distributed independently across $i$ and $t$ with zero mean and variance $\sigma_{i}^{2}>0$. Although the common factors in $\boldsymbol{f}_{t}$ are modelled as unobservable, we can control for these by augmenting the ARDL model (1) with cross-sectional averages of the model's observable variables following the correlated effects augmentation of Pesaran (2006): Averaging (1) across $i$ under the assumption that slope coefficients and regressors are uncorrelated, one obtains

$$
\bar{y}_{t}=\bar{\omega}+\sum_{k=1}^{p} \bar{\rho}_{k} \bar{y}_{t-k}+\sum_{k=0}^{q} \bar{\varrho}_{k}^{\prime} \overline{\boldsymbol{x}}_{t-k}+\overline{\boldsymbol{\lambda}}^{\prime} \boldsymbol{f}_{t}+\bar{\varepsilon}_{t},
$$

\footnotetext{
${ }^{3}$ For simplicity of notation, we denote the lag orders by $p$ and $q$, respectively, although in our empirical implementation we will allow for these to differ across variables and countries, that is, work with the model specification

$$
y_{i t}=\omega_{i}+\sum_{k=1}^{p_{i}} \rho_{i k} y_{i, t-k}+\sum_{\ell=1}^{m} \sum_{k=0}^{q_{\ell i}} \varrho_{\ell i k} x_{\ell i, t-k}+u_{i t} .
$$
}


where $\bar{y}_{t-k}=N^{-1} \sum_{i=1}^{N} y_{i, t-k}, \bar{\rho}_{k}=N^{-1} \sum_{i=1}^{N} \rho_{i k}, k=0,1, \ldots, p ; \bar{\omega}=N^{-1} \sum_{i=1}^{N} \omega_{i} ; \overline{\boldsymbol{x}}_{t-k}=$ $N^{-1} \sum_{i=1}^{N} \boldsymbol{x}_{i, t-k}, \bar{\varrho}_{k}=N^{-1} \sum_{i=1}^{N} \varrho_{i k}, k=0,1, \ldots, q ; \overline{\boldsymbol{\lambda}}=N^{-1} \sum_{i=1}^{N} \boldsymbol{\lambda}_{i}$ and $\bar{\varepsilon}_{t}=N^{-1} \sum_{i=1}^{N} \varepsilon_{i t}$.

Since the error component $\varepsilon_{i t}$ by assumption is independently distributed across $i$ and $t, \bar{\varepsilon}_{t}$ tends to zero in root mean square error as $N$ becomes large. The cross-sectional correlation in $u_{i t}$ can therefore be captured through a linear combination of the cross-sectional averages of the dependent variable and of all regressors:

$$
\boldsymbol{\lambda}_{i}^{\prime} \boldsymbol{f}_{t}=\vartheta_{i} \overline{\boldsymbol{\lambda}}^{\prime} \boldsymbol{f}_{t}=\eta_{i} \bar{y}_{t}+\boldsymbol{\zeta}_{i}^{\prime} \overline{\boldsymbol{x}}_{t}+\sum_{k=0}^{p-1} \nu_{i k} \Delta \bar{y}_{t-k}+\sum_{k=0}^{q-1} \boldsymbol{\varsigma}_{i k}^{\prime} \Delta \overline{\boldsymbol{x}}_{t-k}-\vartheta_{i} \bar{\omega},
$$

with reparameterizations $\eta_{i}=\vartheta_{i}\left(1-\sum_{k=1}^{p} \bar{\rho}_{k}\right), \boldsymbol{\zeta}_{i}=\vartheta_{i}\left(\sum_{k=0}^{q} \overline{\boldsymbol{\varrho}}_{k}\right), \nu_{i k}=\vartheta_{i}\left(\sum_{\ell=k+1}^{p} \bar{\rho}_{\ell}\right)$ and $\boldsymbol{\varsigma}_{i k}=\vartheta_{i}\left(\sum_{\ell=k+1}^{q} \overline{\boldsymbol{\varrho}}_{\ell}\right)$, for some $\vartheta_{i}$. Using Equation (4), the error-correction representation of the panel ARDL model (1) and (2) can be written as:

$$
\begin{array}{r}
\Delta y_{i t}=\mu_{i}+\alpha_{i} y_{i, t-1}+\boldsymbol{\beta}_{i}^{\prime} \boldsymbol{x}_{i t}+\sum_{k=1}^{p-1} \phi_{i k} \Delta y_{i, t-k}+\sum_{k=0}^{q-1} \boldsymbol{\delta}_{i k}^{\prime} \Delta \boldsymbol{x}_{i, t-k} \\
+\eta_{i} \bar{y}_{t}+\boldsymbol{\zeta}_{i}^{\prime} \overline{\boldsymbol{x}}_{t}+\sum_{k=0}^{p-1} \nu_{i k} \Delta \bar{y}_{t-k}+\sum_{k=0}^{q-1} \boldsymbol{\varsigma}_{i k}^{\prime} \Delta \overline{\boldsymbol{x}}_{t-k}+\varepsilon_{i t}
\end{array}
$$

with $\mu_{i}=\omega_{i}-\vartheta_{i} \bar{\omega}, \alpha_{i}=-\left(1-\sum_{k=1}^{p} \rho_{i k}\right), \boldsymbol{\beta}_{i}=\sum_{k=0}^{q} \boldsymbol{\varrho}_{i k}, \phi_{i k}=-\sum_{\ell=k+1}^{p} \rho_{i \ell}$ and $\boldsymbol{\delta}_{i k}=$ $-\sum_{\ell=k+1}^{q} \boldsymbol{\varrho}_{i \ell}$. From (5) the long-run relationship between $y$ and $\boldsymbol{x}$ is given by

$$
y_{i, t-1}^{L R}=-\alpha_{i}^{-1} \boldsymbol{\beta}_{i}^{\prime} \boldsymbol{x}_{i t}-\alpha_{i}^{-1} \mu_{i}-\alpha_{i}^{-1}\left(\eta_{i} \bar{y}_{t}+\boldsymbol{\zeta}_{i}^{\prime} \overline{\boldsymbol{x}}_{t}\right)=\boldsymbol{\theta}_{i}^{\prime} \boldsymbol{x}_{i t}-\alpha_{i}^{-1}\left(\mu_{i}+\boldsymbol{\chi}_{i}^{\prime} \boldsymbol{g}_{t}\right)
$$

where $\boldsymbol{g}_{t}=\left(\bar{y}_{t} \overline{\boldsymbol{x}}_{t}^{\prime}\right)^{\prime}$ represents the level parts of the common factors and $\boldsymbol{\chi}_{i}=\left(\eta_{i} \boldsymbol{\zeta}_{i}^{\prime}\right)^{\prime}$ contains the loadings on these common factors.

The long-run coefficients between $y_{i}$ and $\boldsymbol{x}_{i}$, given by $\boldsymbol{\theta}_{i}$, and the speed of adjustment towards the long-run relation for country $i$, given by $\alpha_{i}$, constitute the key coefficients of economic interest in the panel ARDL model (5). In what follows, we will therefore also work with a transformed version of the model in (5) that only keeps the coefficients in $\left(\alpha_{i} \boldsymbol{\theta}_{i}^{\prime}\right)$, and extracts all other coefficients using their (country-specific) least-squares estimators. Defining the country-specific long-run deviation, $\xi_{i t}$, as follows:

$$
\xi_{i t}\left(\boldsymbol{\theta}_{i}\right)=y_{i, t-1}-\boldsymbol{\theta}_{i}^{\prime} \boldsymbol{x}_{i t},
$$

upon multiplying Equation (5) with an idempotent matrix $\boldsymbol{M}_{i}$, where

$$
\boldsymbol{M}_{i}=\boldsymbol{I}_{T_{i}}-\boldsymbol{H}_{i}\left(\boldsymbol{H}_{i}^{\prime} \boldsymbol{H}_{i}\right)^{-1} \boldsymbol{H}_{i}^{\prime}
$$

with $\boldsymbol{I}_{T_{i}}$ denoting the identity matrix of dimension $T_{i}$ and $\boldsymbol{H}_{i}$ capturing the extracted regressors,

$$
\boldsymbol{H}_{i}=\left(\begin{array}{llll}
\boldsymbol{h}_{i 1} & \boldsymbol{h}_{i 2} & \ldots & \boldsymbol{h}_{i T_{i}}
\end{array}\right)^{\prime},
$$


with

$$
\begin{aligned}
& \boldsymbol{h}_{i t}=\left(\begin{array}{lllllllll}
1 & \Delta y_{i, t-1} & \Delta y_{i, t-2} & \ldots & \Delta y_{i, t-p+1} & \Delta \boldsymbol{x}_{i t}^{\prime} & \Delta \boldsymbol{x}_{i, t-1}^{\prime} & \ldots & \Delta \boldsymbol{x}_{i, t-q+1}^{\prime}
\end{array}\right. \\
& \begin{array}{llllllllll}
\bar{y}_{t} & \overline{\boldsymbol{x}}_{t}^{\prime} & \Delta \bar{y}_{t} & \Delta \bar{y}_{t-1} & \ldots & \Delta \bar{y}_{t-p+1} & \Delta \overline{\boldsymbol{x}}_{t}^{\prime} & \Delta \overline{\boldsymbol{x}}_{t-1}^{\prime} & \ldots & \left.\Delta \overline{\boldsymbol{x}}_{t-q+1}^{\prime}\right)^{\prime},
\end{array}
\end{aligned}
$$

we obtain the transformed model

$$
\boldsymbol{M}_{i} \Delta \boldsymbol{y}_{i}=\alpha_{i}\left(\boldsymbol{M}_{i} \boldsymbol{y}_{i,-1}-\boldsymbol{M}_{i} \boldsymbol{X}_{i} \boldsymbol{\theta}_{i}\right)+\boldsymbol{\varepsilon}_{i}=\alpha_{i} \boldsymbol{M}_{i} \boldsymbol{\xi}_{i}\left(\boldsymbol{\theta}_{i}\right)+\boldsymbol{\varepsilon}_{i},
$$

where for each country we have stacked all variables across time periods, such that

$$
\begin{gathered}
\Delta \boldsymbol{y}_{i}=\left(\begin{array}{llll}
\Delta y_{i 1} & \Delta y_{i 2} & \ldots & \Delta y_{i T_{i}}
\end{array}\right)^{\prime}, \\
\boldsymbol{y}_{i,-1}=\left(\begin{array}{llll}
y_{i 0} & y_{i 1} & \ldots & y_{i, T_{i}-1}
\end{array}\right)^{\prime}, \\
\boldsymbol{X}_{i}=\left(\begin{array}{llll}
\boldsymbol{x}_{i 1} & \boldsymbol{x}_{i 2} & \ldots & \boldsymbol{x}_{i T_{i}}
\end{array}\right)^{\prime}, \\
\boldsymbol{\xi}_{i}\left(\boldsymbol{\theta}_{i}\right)=\left[\begin{array}{llll}
\xi_{i 1}\left(\boldsymbol{\theta}_{i}\right) & \xi_{i 2}\left(\boldsymbol{\theta}_{i}\right) & \ldots & \xi_{i T_{i}}\left(\boldsymbol{\theta}_{i}\right)
\end{array}\right]^{\prime},
\end{gathered}
$$

and

$$
\boldsymbol{\varepsilon}_{i}=\left(\begin{array}{llll}
\varepsilon_{i 1} & \varepsilon_{i 2} & \ldots & \varepsilon_{i T_{i}}
\end{array}\right)^{\prime}
$$

The Pesaran and Smith (1995) MG estimators of $\alpha_{i}$ and $\boldsymbol{\theta}_{i}$ are obtained by least-squares estimation of (10) for each country separately and subsequently averaging the countryspecific coefficient estimates. Standard errors for these MG estimates can be computed non-parametrically on the basis of the spread of the coefficients across countries.

The idea underlying the Pesaran, Shin and Smith (1999) PMG estimation is to assume that the long-run coefficients $\boldsymbol{\theta}_{i}$ are homogeneous across all countries (that is, $\boldsymbol{\theta}_{i}=\boldsymbol{\theta}, i=$ $1,2, \ldots, N)$, but that all other coefficients are still allowed to differ in unrestricted fashion across countries. The PMG estimator is based on numerical maximization of the implied restricted likelihood function.

\subsection{Conditioning the Pooled Mean Group Approach: The CPMG Model}

The PMG estimator exhibits considerable appeal for the study of exchange rate dynamics: It is rather unlikely that the short-run dynamics of nominal exchange rates and domestic as well as foreign prices exhibit strong commonalities across countries - it thus appears to be a very sensible choice to let such short-run dynamics differ in unconstrained fashion across countries, as the PMG estimator does do. At the same time, the PPP hypothesis imposes a common restriction across countries on the long-run coefficients, that the PMG estimator does incorporate. 
As we have argued in the Introduction, though, it seems unlikely that PPP would hold even in the long run across all countries and their differing macroeconomic and financial environments. To capture the interaction between medium- to long-run exchange rate dynamics on the one hand and a country's international investment position on the other hand, we propose to condition the coefficients in the long-run relation between nominal exchange rates and domestic as well as foreign prices on a predetermined state variable measuring a country's international investment position. To map this idea back to the generic panel ARDL model (5), denoting the value of the conditioning predetermined state variable by $\tilde{z}_{i t}$, ${ }^{4}$ we therefore propose the following augmented model:

$$
\begin{aligned}
\Delta y_{i t}=\mu_{i}+ & \alpha_{i}\left(\tilde{z}_{i t}\right) y_{i, t-1}+\boldsymbol{\beta}_{i}\left(\tilde{z}_{i t}\right)^{\prime} \boldsymbol{x}_{i t}+\sum_{k=1}^{p-1} \phi_{i k}\left(\tilde{z}_{i t}\right) \Delta y_{i, t-k}+\sum_{k=0}^{q-1} \boldsymbol{\delta}_{i k}\left(\tilde{z}_{i t}\right)^{\prime} \Delta \boldsymbol{x}_{i, t-k} \\
& +\eta_{i}\left(\tilde{z}_{i t}\right) \bar{y}_{t}+\boldsymbol{\zeta}_{i}\left(\tilde{z}_{i t}\right)^{\prime} \overline{\boldsymbol{x}}_{t}+\sum_{k=0}^{p-1} \nu_{i k}\left(\tilde{z}_{i t}\right) \Delta \bar{y}_{t-k}+\sum_{k=0}^{q-1} \boldsymbol{\varsigma}_{i k}\left(\tilde{z}_{i t}\right)^{\prime} \Delta \overline{\boldsymbol{x}}_{t-k}+\varepsilon_{i t},
\end{aligned}
$$

where, in analogy to (6), we have

$$
\alpha_{i}\left(\tilde{z}_{i t}\right) y_{i, t-1}+\boldsymbol{\beta}_{i}\left(\tilde{z}_{i t}\right)^{\prime} \boldsymbol{x}_{i t}=\alpha_{i}\left(\tilde{z}_{i t}\right)\left[y_{i, t-1}-\boldsymbol{\theta}\left(\tilde{z}_{i t}\right)^{\prime} \boldsymbol{x}_{i t}\right]
$$

Note that all short-run coefficients in (11) are a function of both $\tilde{z}_{i t}$ as well as other countryspecific characteristics (reflected in the $i$ subscripts for all coefficient functionals), but that the long-run coefficients in (12) are specified across all countries as a homogeneous function of $\tilde{z}_{i t}: \boldsymbol{\theta}_{i}\left(\tilde{z}_{i t}\right)=\boldsymbol{\theta}\left(\tilde{z}_{i t}\right), i=1,2, \ldots, N$.

We propose to specify $\boldsymbol{\theta}\left(\tilde{z}_{i t}\right)$ using a parametric function of flexible form, and in particular choose Chebyshev polynomials as one specification of orthogonal polynomials. ${ }^{5}$ Our CPMG model specifies that

$$
\boldsymbol{\theta}\left(\tilde{z}_{i t}\right)=\sum_{s=0}^{\tau} \boldsymbol{\gamma}_{s}^{(\boldsymbol{\theta})} \cdot c_{s}\left(\tilde{z}_{i t}\right),
$$

with the Chebyshev polynomials $c_{s}\left(\tilde{z}_{i t}\right)$ recursively defined as

$$
c_{s+1}\left(\tilde{z}_{i t}\right)=2 \tilde{z}_{i t} c_{s}\left(\tilde{z}_{i t}\right)-c_{s-1}\left(\tilde{z}_{i t}\right), \quad s=1,2, \ldots, \tau,
$$

$c_{0}\left(\tilde{z}_{i t}\right)=1$ and $c_{1}\left(\tilde{z}_{i t}\right)=\tilde{z}_{i t}$, and where $\boldsymbol{\gamma}_{s}^{(\boldsymbol{\theta})}$ is an $m$-dimensional vector of coefficients that is homogeneous across countries. The coefficient functionals $\alpha_{i}\left(\tilde{z}_{i t}\right), \phi_{i k}\left(\tilde{z}_{i t}\right)$ etc. can be specified in similar form (albeit with country-specific rather than homogeneous coefficients). One approach to the estimation of the CPMG model is to concentrate the likelihood function, writing it as a function of $\alpha_{i}\left(\tilde{z}_{i t}\right)$ and $\boldsymbol{\theta}\left(\tilde{z}_{i t}\right)$ (the coefficient functions of economic interest)

\footnotetext{
${ }^{4}$ In this paper, we specify $\tilde{z}_{i t}$ to be a scalar. The extension to considering a vector of state variables is beyond the scope of this paper and is left for future research.

${ }^{5}$ We work with orthogonal polynomials in part as an effective means to avoid multicollinearity problems.
} 
only, and subsequently maximize this concentrated likelihood function. A computationally less burdensome alternative that we focus on in this paper is to adapt the two-step estimation strategy proposed by Breitung (2005) for the PMG model to our CPMG model: Rewriting (11) as

$$
\Delta y_{i t}=y_{i, t-1} \alpha_{i}\left(\tilde{z}_{i t}\right)+\boldsymbol{x}_{i t}^{\prime} \boldsymbol{\beta}_{i}\left(\tilde{z}_{i t}\right)+\boldsymbol{h}_{i t}^{\prime} \boldsymbol{\psi}_{i}\left(\tilde{z}_{i t}\right)+\varepsilon_{i t}, \quad i=1,2, \ldots, N, t=1,2, \ldots, T_{i},
$$

where

$$
\begin{aligned}
& \boldsymbol{\psi}_{i}\left(\tilde{z}_{i t}\right)=\left[\begin{array}{lllllllll}
\mu_{i}\left(\tilde{z}_{i t}\right) & \phi_{i 1}\left(\tilde{z}_{i t}\right) & \phi_{i 2}\left(\tilde{z}_{i t}\right) & \ldots & \phi_{i, p-1}\left(\tilde{z}_{i t}\right) & \boldsymbol{\delta}_{i 0}\left(\tilde{z}_{i t}\right)^{\prime} & \boldsymbol{\delta}_{i 1}\left(\tilde{z}_{i t}\right)^{\prime} & \ldots & \boldsymbol{\delta}_{i, q-1}\left(\tilde{z}_{i t}\right)^{\prime}
\end{array}\right. \\
& \left.\eta_{i}\left(\tilde{z}_{i t}\right) \boldsymbol{\zeta}_{i}\left(\tilde{z}_{i t}\right)^{\prime} \quad \nu_{i 0}\left(\tilde{z}_{i t}\right) \quad \nu_{i 1}\left(\tilde{z}_{i t}\right) \quad \ldots \quad \nu_{i, p-1}\left(\tilde{z}_{i t}\right) \boldsymbol{\varsigma}_{i 0}\left(\tilde{z}_{i t}\right)^{\prime} \boldsymbol{\varsigma}_{i 1}\left(\tilde{z}_{i t}\right)^{\prime} \quad \ldots \quad \boldsymbol{\varsigma}_{i, q-1}\left(\tilde{z}_{i t}\right)^{\prime}\right]^{\prime},
\end{aligned}
$$

$\boldsymbol{\psi}_{i}\left(\tilde{z}_{i t}\right)$ denoting the (state-dependent) coefficient vector on the variables that are neither relevant for country $i$ 's long-run relationship nor its speed of adjustment, in the first step we estimate the coefficients in (14) (including $\sigma_{i}^{2}$ and estimating $\boldsymbol{\beta}_{i}\left(\tilde{z}_{i t}\right)$ rather than $\boldsymbol{\theta}\left(\tilde{z}_{i t}\right)$, so that all estimated coefficients are country-specific) from

$$
\Delta \boldsymbol{y}_{i}=\mathcal{Y}_{i,-1}\left(\tilde{\boldsymbol{z}}_{i}\right) \boldsymbol{\gamma}_{i}^{\left(\alpha_{i}\right)}+\boldsymbol{\mathcal { X }}_{i}\left(\tilde{\boldsymbol{z}}_{i}\right) \boldsymbol{\gamma}_{i}^{\left(\boldsymbol{\beta}_{i}\right)}+\boldsymbol{\mathcal { H }}_{i}\left(\tilde{\boldsymbol{z}}_{i}\right) \boldsymbol{\gamma}_{i}^{\left(\boldsymbol{\psi}_{i}\right)}+\boldsymbol{\varepsilon}_{i}
$$

where $\mathcal{Y}_{i,-1}\left(\tilde{\boldsymbol{z}}_{i}\right), \boldsymbol{\mathcal { X }}_{i}\left(\tilde{\boldsymbol{z}}_{i}\right)$ and $\boldsymbol{\mathcal { H }}_{i}\left(\tilde{\boldsymbol{z}}_{i}\right)$ are combinations of $\boldsymbol{y}_{i,-1}, \boldsymbol{X}_{i}$ and $\boldsymbol{H}_{i}$, respectively, with the Chebyshev polynomials, and $\boldsymbol{\gamma}_{i}^{\left(\alpha_{i}\right)}, \boldsymbol{\gamma}_{i}^{\left(\boldsymbol{\beta}_{i}\right)}$ and $\boldsymbol{\gamma}_{i}^{\left(\boldsymbol{\psi}_{i}\right)}$ are the polynomial coefficients. For a detailed description of the matrices of coefficients and variables involved see Appendix A. This first step can be accomplished using country-specific least squares. In a second step, estimate the conditionally homogeneous long-run coefficients through pooled least-squares estimation of a transformed model concentrating out all country-specific coefficients, namely,

$$
\boldsymbol{v}_{i}=-\mathcal{X}_{i}\left(\tilde{\boldsymbol{z}}_{i}\right) \boldsymbol{\gamma}^{(\boldsymbol{\theta})}+\boldsymbol{\epsilon}_{i}
$$

where

$$
\begin{gathered}
\boldsymbol{v}_{i}=\hat{\boldsymbol{A}}_{i}\left(\tilde{\boldsymbol{z}}_{i}\right)^{-1}\left[\begin{array}{lll}
\Delta \boldsymbol{y}_{i}-\boldsymbol{\mathcal { H }}_{i}\left(\tilde{\boldsymbol{z}}_{i}\right) \hat{\boldsymbol{\gamma}}_{i}^{\left(\boldsymbol{\psi}_{i}\right)}
\end{array}\right]-\boldsymbol{y}_{i,-1}, \\
\boldsymbol{\gamma}^{(\boldsymbol{\theta})}=\left[\begin{array}{llll}
\gamma_{0}^{(\boldsymbol{\theta})^{\prime}} & \gamma_{1}^{(\boldsymbol{\theta})^{\prime}} & \ldots & \boldsymbol{\gamma}_{\tau}^{(\boldsymbol{\theta})^{\prime}}
\end{array}\right]^{\prime},
\end{gathered}
$$

(recalling from (13) that $\boldsymbol{\theta}\left(\tilde{z}_{i t}\right)=\sum_{s=0}^{\tau} \boldsymbol{\gamma}_{s}^{(\boldsymbol{\theta})} \cdot c_{s}\left(\tilde{z}_{i t}\right)$ ),

$$
\boldsymbol{\epsilon}_{i}=\hat{\boldsymbol{A}}_{i}\left(\tilde{\boldsymbol{z}}_{i}\right)^{-1} \varepsilon_{i}
$$

and

$$
V\left(\boldsymbol{\epsilon}_{i}\right)=\hat{\boldsymbol{A}}_{i}\left(\tilde{\boldsymbol{z}}_{i}\right)^{-2} \hat{\sigma}_{i}^{2},
$$

with $\boldsymbol{A}_{i}\left(\tilde{\boldsymbol{z}}_{i}\right)=\operatorname{diag}\left[\alpha_{i}\left(\tilde{z}_{i 1}\right), \alpha_{i}\left(\tilde{z}_{i 2}\right), \ldots, \alpha_{i}\left(\tilde{z}_{i T}\right)\right]$. Equation $(16)$ is derived in Appendix A. 
In practice, to keep the model structure parsimonious one may wish to restrict the orders of most polynomials in (11) (except for those in $y_{i, t-1}$ and $\boldsymbol{x}_{i t}$ ) to zero. Note that such a restriction is completely consistent with the idea of unrestricted cross-country heterogeneity of the model's short-run dynamics. Due to the heterogeneous nature of the functional relationship between $\alpha_{i}$ and the conditioning variable $\tilde{z}_{i t}$ that still remains under such a restriction, we should be explicit about how we propose to compute a panel estimate of the speed of adjustment coefficient for each value of the conditioning state variable, $\tilde{z}_{i t}$. For each $\tilde{z}_{i t}$ we compute the average across all functionals $\alpha_{j}\left(\tilde{z}_{i t}\right), j=1,2, \ldots, N$, incorporating in the averaging procedure a weighting with respect to the local environment for which each $\alpha_{j}\left(\tilde{z}_{i t}\right)$ has been estimated. The details of the procedure we use to compute a smoothed mean group estimate of the speed of adjustment coefficient and its corresponding standard error are laid out in Appendix B.

\subsection{Non-Parametric Conditioning: The SKMG Model}

The CPMG model carefully separates the form of the effect of changes in the conditioning state variable $\tilde{z}_{i t}$ on speed of adjustment/short-run coefficients (through country-specific

conditioning functions) from those on the long-run coefficients (through pooled conditioning functions). An alternative conditioning procedure would be to make the form of the conditioning dependent on the specific value that the conditioning state variable assumes; that is, to construct conditioning functions that do not differ across short- vs. long-run coefficients, but for both types of coefficients give priority to "neighboring" values of the conditioning state variable, and assign more distant values of the conditioning state variable a relatively minor role in shaping the conditioning functions. To pursue this latter idea, our SKMG model introduces a non-parametric kernel for the panel ARDL model (1). Building on the work of Kumar and Ullah (2000), we weight all available observations using a kernel function and minimize a modified residual sum of squares, namely

$$
\hat{\wp}\left(\tilde{z}_{j s}\right)=\underset{\wp\left(\tilde{z}_{j s}\right)}{\operatorname{argmin}} \sum_{i=1}^{N} \sum_{t=1}^{T_{i}} \varepsilon_{i t}^{2} \kappa\left(\tilde{z}_{i t}-\tilde{z}_{j s}\right), \quad j=1,2, \ldots, N, \quad s=1,2, \ldots, T_{i},
$$

where $\wp\left(\tilde{z}_{j s}\right)=\left[\alpha\left(\tilde{z}_{j s}\right) \boldsymbol{\beta}\left(\tilde{z}_{j s}\right)^{\prime} \boldsymbol{\psi}\left(\tilde{z}_{j s}\right)^{\prime}\right]^{\prime}$ comprises the local values of the coefficients in Equation (14) and $\kappa\left(\tilde{z}_{i t}-\tilde{z}_{j s}\right)$ represents the kernel that effectively gives higher weight to observations "close" to $\tilde{z}_{j s}$ and lower weight to observations "far" from this point.

Adhering as for the CPMG model to the principle of parsimony and only incorporating kernels for the coefficients on $y_{i, t-1}$ and $\boldsymbol{x}_{i t}$, the concentrated version of our panel ARDL model (11), stacked in time dimension, becomes:

$$
\Delta \boldsymbol{y}_{i}^{*}=\boldsymbol{y}_{i,-1}^{*} \alpha\left(\tilde{z}_{j s}\right)+\boldsymbol{X}_{i}^{*} \boldsymbol{\beta}\left(\tilde{z}_{j s}\right)+\boldsymbol{\varepsilon}_{i}, \quad j=1,2, \ldots, N, \quad s=1,2, \ldots, T_{i} .
$$


with $\Delta \boldsymbol{y}_{i}^{*}=\boldsymbol{M}_{i} \Delta \boldsymbol{y}_{i}, \boldsymbol{y}_{i,-1}^{*}=\boldsymbol{M}_{i} \boldsymbol{y}_{i,-1}$, and $\boldsymbol{X}_{i}^{*}=\boldsymbol{M}_{i} \boldsymbol{X}_{i}$. Taking account of heteroskedastic variances $\sigma_{i}^{2}$, Equation (17) can be solved using the Local Least Squares Kernel (LLSK) estimator,

$$
\hat{\boldsymbol{\varphi}}\left(\tilde{z}_{j s}\right)=\left[\boldsymbol{W}^{\prime} \boldsymbol{\Omega}^{-1}\left(\tilde{z}_{j s}\right) \boldsymbol{W}\right]^{-1} \boldsymbol{W}^{\prime} \boldsymbol{\Omega}^{-1}\left(\tilde{z}_{j s}\right) \Delta \boldsymbol{y}^{*}, \quad j=1,2, \ldots, N, s=1,2, \ldots, T_{i},
$$

where

$$
\begin{aligned}
& \boldsymbol{\varphi}\left(\tilde{z}_{j s}\right)=\left[\alpha\left(\tilde{z}_{j s}\right) \boldsymbol{\beta}\left(\tilde{z}_{j s}\right)^{\prime}\right]^{\prime}, \\
& \boldsymbol{W}=\left(\boldsymbol{y}_{-1}^{*} \boldsymbol{X}^{*}\right), \\
& \boldsymbol{\Omega}^{-1}\left(\tilde{z}_{j s}\right)=\boldsymbol{\Omega}^{-1 / 2} \boldsymbol{K}\left(\tilde{z}_{j s}\right) \boldsymbol{\Omega}^{-1 / 2},
\end{aligned}
$$

with $\Delta \boldsymbol{y}^{*}=\left(\Delta \boldsymbol{y}_{1}^{* \prime} \Delta \boldsymbol{y}_{2}^{* \prime} \ldots \Delta \boldsymbol{y}_{N}^{* \prime}\right)^{\prime}, \boldsymbol{y}_{-1}^{*}=\left(\boldsymbol{y}_{1,-1}^{*}{ }^{\prime} \boldsymbol{y}_{2,-1}^{*} \ldots \boldsymbol{y}_{N,-1}^{*}\right)^{\prime}$ and $\boldsymbol{X}^{*}=\left(\boldsymbol{X}_{1}^{* \prime} \boldsymbol{X}_{2}^{* \prime} \ldots \boldsymbol{X}_{N}^{* \prime}\right)^{\prime}$. $\boldsymbol{K}\left(\tilde{z}_{j s}\right)$ is a diagonal matrix containing the values of $\kappa\left(\tilde{z}_{i t}-\tilde{z}_{j s}\right)$; for its actual shape see Appendix C. The variance matrix $\Omega$ is defined as

$$
\boldsymbol{\Omega}=\operatorname{diag}\left(\sigma_{1}^{2}, \sigma_{2}^{2}, \ldots, \sigma_{N}^{2}\right) \otimes \boldsymbol{I}_{T_{i}},
$$

and can be estimated using OLS estimates of $\sigma_{i}^{2}$ for each country. The variance of the parameter estimates can be obtained as

$$
V\left[\hat{\boldsymbol{\varphi}}\left(\tilde{z}_{j s}\right)\right]=\left[\boldsymbol{W}^{\prime} \boldsymbol{\Omega}^{-1}\left(\tilde{z}_{j s}\right) \boldsymbol{W}\right]^{-1} \boldsymbol{W}^{\prime} \boldsymbol{\Omega}_{1}^{-1}\left(\tilde{z}_{j s}\right) \boldsymbol{W}\left[\boldsymbol{W}^{\prime} \boldsymbol{\Omega}^{-1}\left(\tilde{z}_{j s}\right) \boldsymbol{W}\right]^{-1}
$$

where $\boldsymbol{\Omega}_{1}^{-1}\left(\tilde{z}_{j s}\right)=\boldsymbol{\Omega}^{-1 / 2} \boldsymbol{K}^{2}\left(\tilde{z}_{j s}\right) \boldsymbol{\Omega}^{-1 / 2}$.

To allow for richer patterns of coefficient variation across values of the conditioning state variable than obtained by the LLSK estimator, for our SKMG model we incorporate polynomials of higher order into the conditioning procedure as employed in static regression settings by Fan and Zhang (1999). To incorporate the polynomials in the computation of the local coefficients, we again make use of Chebyshev polynomials. We therefore modify the regressors in (19) as follows:

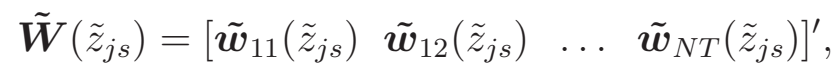

where

$$
\tilde{\boldsymbol{w}}_{i t}\left(\tilde{z}_{j s}\right)=\left[\begin{array}{llll}
\tilde{\boldsymbol{w}}_{1, i t}^{\prime}\left(\tilde{z}_{j s}\right) & \tilde{\boldsymbol{w}}_{2, i t}^{\prime}\left(\tilde{z}_{j s}\right) & \ldots & \tilde{\boldsymbol{w}}_{m+1, i t}^{\prime}\left(\tilde{z}_{j s}\right)
\end{array}\right]^{\prime}
$$

and

$$
\begin{aligned}
& \tilde{\boldsymbol{w}}_{\ell, i t}^{\prime}\left(\tilde{z}_{j s}\right)=w_{\ell, i t}\left[c_{0}\left(\tilde{z}_{i t}-\tilde{z}_{j s}\right) \quad c_{1}\left(\tilde{z}_{i t}-\tilde{z}_{j s}\right) c_{2}\left(\tilde{z}_{i t}-\tilde{z}_{j s}\right) \quad \ldots \quad c_{\tau}\left(\tilde{z}_{i t}-\tilde{z}_{j s}\right)\right] \\
& =w_{\ell, i t} \boldsymbol{\pi}_{\tau}^{\prime}\left(\tilde{z}_{i t}-\tilde{z}_{j s}\right), \quad \ell=1,2, \ldots, m+1 .
\end{aligned}
$$

Note that $w_{\ell, i t}$ refers to observation $(i, t)$ for the $\ell$-th variable in $\boldsymbol{W}$. We denote the estimator that results from the right-hand side of (19), but with $\boldsymbol{W}$ replaced by $\tilde{\boldsymbol{W}}$, as $\hat{\tilde{\varphi}}_{\ell}\left(\tilde{z}_{j s}\right)$. This estimator can in turn be used to construct interpolated estimates of $\varphi\left(\tilde{z}_{j s}\right), \hat{\varphi}\left(\tilde{z}_{j s}\right)$, as

$$
\hat{\varphi}_{\ell}\left(\tilde{z}_{j s}\right)=\frac{\sum_{i=1}^{N} \sum_{t=1}^{T} \boldsymbol{\pi}_{\tau}^{\prime}\left(\tilde{z}_{j s}-\tilde{z}_{i t}\right) \hat{\tilde{\varphi}}_{\ell}\left(\tilde{z}_{i t}\right) \kappa\left(\tilde{z}_{j s}-\tilde{z}_{i t}\right)}{\sum_{i=1}^{N} \sum_{t=1}^{T} \kappa\left(\tilde{z}_{j s}-\tilde{z}_{i t}\right)}, \quad \ell=1,2, \ldots, m+1,
$$


and

$V\left[\hat{\tilde{\varphi}}_{\ell}\left(\tilde{z}_{j s}\right)\right]=\frac{\sum_{i=1}^{N} \sum_{t=1}^{T} \boldsymbol{\pi}_{\tau}^{\prime}\left(\tilde{z}_{j s}-\tilde{z}_{i t}\right) V\left[\hat{\tilde{\boldsymbol{\varphi}}}_{\ell}\left(\tilde{z}_{i t}\right)\right] \boldsymbol{\pi}_{\tau}\left(\tilde{z}_{j s}-\tilde{z}_{i t}\right) \kappa\left(\tilde{z}_{j s}-\tilde{z}_{i t}\right)}{\sum_{i=1}^{N} \sum_{t=1}^{T} \kappa\left(\tilde{z}_{j s}-\tilde{z}_{i t}\right)}, \quad \ell=1,2, \ldots, m+1$.

We call the resultant estimator the SKMG estimator.

Contrasting the ideas underlying the CPMG and SKMG modelling approaches, the parametric CPMG is the more parsimonious of the two approaches. However, it also tends to be the less robust of the two approaches, as the curvatures of the conditioning functions can be more heavily influenced by outlying values of the conditioning variable. All modelling approaches, MG, PMG, CPMG and SKMG, require the existence of a long-run relation between $y_{i t}$ and $\boldsymbol{x}_{i t}$ which has to be tested for prior to the application of the estimation procedures. Appendix D reviews the panel cointegration test of Westerlund (2005), which we will employ in the empirical part of our paper, and its applicability both for models with unconditional and conditional long-run relations.

\section{International Capital Flow and Investment Position Data}

We have assembled a new database for this paper featuring data on international capital flows and the implied international investment positions of countries. Our database comprises these data on an annual basis for a total of 153 countries over the time period 1970 to 2004 . We obtained most of the flow data from the International Monetary Fund's (IMF) Balance of Payments Statistics (BOPS); stock data were taken from the IMF's International Investment Position (IIP) database as well as the World Bank's Global Development Finance (GDF) database. All international capital data we used were compiled in millions of U.S. Dollars. In addition to international capital flows and stocks, our database incorporates data on gross domestic product (GDP) from the World Bank's World Development Indicators database, ${ }^{6}$ bilateral nominal exchange rates and consumer prices from the IFS, as well as exports and imports which are taken from the Direction of Trade Statistics also maintained by the IMF. The key difficulty in the compilation of our database was that the IIP for most countries only contains a small number of observations. It was therefore essential to augment the IIP stock data by cumulating flow data. For this cumulation the stock data have to be initialized with an existing stock figure for some reference period. For the overall investment position of a country, the NFA position, possibly the best source of such a figure is Sinn (1990) who provides NFA estimates for up to 145 countries over the period 1970 to 1987. For the subcomponents of NFA we used stock data from the IIP database for purposes of initialization.

\footnotetext{
${ }^{6}$ Some of the GDP data in this database are reported in domestic currency values; we converted such GDP data to U.S. Dollar figures using yearly average bilateral exchange rates.
} 
Given that the flow data may have an earlier starting point than the stock data, occasionally we needed to backcast the initial stock value. In effect, our cumulative flow figures are thus anchored by the first available stock figure from IIP data. We did not compute cumulative flow figures if they did not overlap with corresponding stock data.

Changes in the stock of any asset or liability are not only due to new flows, but can also be due to changes in the value of the existing stock. The sources of valuation changes differ across types of financial assets and liabilities. In particular, we adjusted portfolio equity investment liabilities using domestic stock market indices adjusted for exchange rate changes (obtained from Datastream that in turn draws upon Morgan Stanley and other sources) and portfolio equity investment assets using a world stock market index (MSCI World Index from Morgan Stanley). Furthermore, we adjusted foreign direct investment (FDI) liabilities using bilateral real exchange rates relative to the United States, and FDI assets using effective real exchange rates. ${ }^{7}$ Changes in the value of external debt are already incorporated in the stock values reported in the GDF database, and changes in the value of international reserve assets were obtained from the difference between flows and the change in the corresponding stock value. See Appendix E for further details. Denoting net valuation changes aggregated across all asset and liability types as $\Delta N V$, we finally obtained the stock of NFA as

$$
N F A_{i t}=N F A_{i, t-1}+C A_{i t}+K A_{i t}+\Delta N V_{i t},
$$

where $C A_{i t}$ denotes country $i$ 's current account balance at time $t$ and $K A_{i t}$ refers to its capital account balance. ${ }^{8}$

Since we completed compilation of our database, Lane and Milesi-Ferretti (2006) have augmented the international capital flow and investment position database described in Lane and Milesi-Ferretti (2001); the new version of the Lane and Milesi-Ferretti database now has similar cross-country and time coverage as our database. In contrast to Lane and MilesiFerretti (2006), our database also separately reports the valuation effects. For more details on the construction of our database, see Offermanns and Pramor (2006).

\footnotetext{
7 Throughout this paper we use effective exchange rates computed using trade weights. Denoting by $e_{i j t}$ the nominal spot exchange rate between country $i$ and country $j$ (units of country $i$ currency per unit of country $j$ currency), measured as annual averages, we compute the effective exchange rate as $e_{i t}=$ $\sum_{j=1}^{N} \tilde{w}_{i j t} e_{i j t}$. The weights $\tilde{w}_{i j t}$ are computed as predetermined moving averages of country $i$ 's trade volume with country $j$ as a share of country $i$ 's overall trade volume, that is $\tilde{w}_{i j t}=1 / r \cdot \sum_{s=t-r}^{t-1} w_{i j s}$ with $w_{i j t}=$ $\left(E X P_{i j t}+I M P_{i j t}\right) /\left(\sum_{k=1}^{N} E X P_{i k t}+I M P_{i k t}\right)$, where $E X P_{i j t}$ and $I M P_{i j t}$ denote country $i$ 's exports to and imports from country $j$ in U.S. Dollars, and the window width is chosen as $r=3$. While a mixture of trade and capital weights might be most appealing, we have to restrict our attention to trade weights, as information on bilateral flows of capital that would be needed to compute informative capital weights at present is not available for (even a substantial sub-sample of) the broad cross section of countries we wish to examine.

${ }^{8}$ According to the definitions laid out in the fifth edition of the Balance of Payments Manual (BOPM), the sum of the current account balance and the capital account balance offset what is called the financial account balance. Some of the literature still refers to what the BOPM labels as the capital account balance as "net capital transfers" (within the current account), reserving the term "capital account balance" for the change in NFA that we are aiming at.
} 
While our overall database contains annual observations on a total of 153 countries, for the empirical analysis of this paper we restrict attention to 71 countries only. These countries were selected from the 153 countries in our database on the basis of the following criteria:

(i) at least 25 consecutive time-series observations available for all variables entering our analysis;

(ii) population size of at least one million in $1970 ;^{9}$

(iii) economy not centrally planned for (most of) the sample period (according to the classification used by Hall and Jones, 1999);

(iv) economy not a major oil producer (according to the classification used by Mankiw, Romer and Weil, 1992).

The resultant 71 countries included in our analysis are: Algeria, Argentina, Australia, Austria, Bolivia, Brazil, Burkina Faso, Cameroon, Canada, Central African Republic, Chile, Colombia, Costa Rica, Côte d'Ivoire, Denmark, Dominican Republic, Ecuador, Egypt, Finland, France, Germany, Ghana, Greece, Guatemala, Haiti, Honduras, India, Israel, Italy, Jamaica, Japan, Jordan, Kenya, Korea, Libya, Madagascar, Malawi, Malaysia, Mexico, Morocco, Myanmar, Netherlands, New Zealand, Niger, Nigeria, Norway, Pakistan, Panama, Papua New Guinea, Paraguay, Peru, Philippines, Portugal, El Salvador, Senegal, Sierra Leone, Singapore, Spain, Sri Lanka, Sudan, Sweden, Switzerland, Syrian Arab Republic, Thailand, Togo, Turkey, Uganda, United Kingdom, United States, Uruguay and Venezuela. The international investment position data for this set of countries exhibit a highly pronounced increase in the magnitude of gross asset and liability stocks as a ratio to GDP over our sample period. This fact reflects the global trend of increasing international financial integration over the last couple of decades that has led to a massive build-up of international gross capital positions. A breakdown of these positions, defined as the sum of gross asset and liability stocks as a ratio to GDP, shows that among the three major categories of international capital positions, namely foreign direct investment (FDI), equity and debt, FDI positions have risen between 1970 and 2004 by 360\%, equity positions by $880 \%$ and debt positions by $440 \%$, amounting to an overall increase (including that of official reserves) by $450 \%$ (see Figure 1). However, it is not only gross international capital positions that have increased in such astonishing fashion. The process of international financial integration also has had an impact on countries' net international investment positions, resulting in a marked increase of imbalances in net international capital positions. As one measure, the cross-country dispersion of the NFA to GDP ratio has increased by $84 \%$ over our sample period (see Figure 2). As we wish to examine to what extent imbalances in a country's international investment position induce corrections towards PPP as a foreign exchange market anchor, in what follows we will focus on net, not gross, international investment positions. ${ }^{10}$

\footnotetext{
${ }^{9}$ The population data were taken from the World Bank's World Development Indicators, complemented by data from the Penn World Tables.

${ }^{10}$ Our figure confirms the well-documented "world NFA discrepancy" (see, for example, Lane and Milesi-
} 


\section{Empirical Analysis}

\subsection{Model Specification}

To facilitate discussion of our empirical results, let us adapt the generic notation used in Section 3 for our panel ARDL model to the exchange rate model that we take to the data. Based on our general panel ARDL model (5) we specify:

$$
\begin{aligned}
\Delta e_{i t}=\mu_{i} & +\alpha_{i}\left(\tilde{z}_{i t}\right)\left[e_{i, t-1}-\boldsymbol{\theta}_{i}\left(\tilde{z}_{i t}\right)^{\prime}\left(\begin{array}{c}
p_{i t} \\
p_{i t}^{*}
\end{array}\right)\right]+\eta_{i} \bar{e}_{t}+\zeta_{i}\left(\bar{p}_{t}-\bar{p}_{t}^{*}\right) \\
& +\sum_{k=1}^{p_{i}-1} \phi_{i k} \Delta e_{i, t-k}+\sum_{k=0}^{q_{1 i}-1} \delta_{1 i k} \Delta p_{i, t-k}+\sum_{k=0}^{q_{2 i}-1} \delta_{2 i k} \Delta p_{i, t-k}^{*} \\
& +\sum_{k=0}^{p_{i}-1} \nu_{i k} \Delta \bar{e}_{t-k}+\sum_{k=0}^{\max \left(q_{1 i}, q_{2 i}\right)-1} \varsigma_{i k}\left(\Delta \bar{p}_{t-k}-\Delta \bar{p}_{t-k}^{*}\right)+\varepsilon_{i t},
\end{aligned}
$$

where $e_{i t}$ denotes the logarithm of country $i$ 's effective nominal spot exchange rate, $p_{i t}$ the logarithm of country $i$ 's consumer price index and $p_{i t}^{*}$ the logarithm of weighted foreign consumer price indices (using the same weighting scheme as for the effective exchange rate). ${ }^{11}$ Note that the PPP hypothesis does not pin down a unique choice of dependent and independent variables for the ARDL model. We specify the effective nominal exchange rate as the dependent variable, as our primary interest is in how the nominal and real exchange rates adjust to changes of macroeconomic and financial fundamentals. Our choice of the dependent variable does not imply that we are assuming domestic and weighted foreign prices to be (strictly) exogenous, however. In the context of ARDL models endogeneity of an independent variable can be overcome by adding sufficiently many lags of that independent variable. ${ }^{12}$ To account for the presence of global shocks, following our discussion in Section 3 we augment the model by incorporating cross-sectional averages of the observable variables, denoted by $\bar{e}_{t}, \bar{p}_{t}$ and $\bar{p}_{t}^{*}$, respectively.

The parameters of principal interest are those that have immediate structural interpretation, namely the long-run coefficients $\boldsymbol{\theta}_{i}\left(\tilde{z}_{i t}\right)$ and the speed of adjustment parameter $\alpha_{i}\left(\tilde{z}_{i t}\right)$. Note that (unconditional) PPP implies that $\theta_{1 i}=1$ and $\theta_{2 i}=-1$ with $\alpha_{i}<0$. By conditioning these coefficients on $\tilde{z}_{i t}$, we render these dependent on the country's international investment position, specifically on a moving average of the NFA to GDP ratio over the preceding ten

Ferretti, 2006) with a ratio of aggregate NFA to aggregate GDP that averages at -0.058 over our sample period. For the full data set of 153 countries in our database, the ratio of aggregate NFA to aggregate GDP averages at -0.048 over our sample period.

${ }^{11} \mathrm{As}$ is well known, the use of aggregate price indices implies that the long-run relationship, even if consistent with the PPP hypothesis, can only be interpreted as providing evidence for relative (but not absolute) PPP.

${ }^{12}$ For a more detailed discussion of this issue in the time-series setting see Pesaran and Shin (1999). 
years in the sample. This specification of $\tilde{z}_{i t}$ ensures that it can be treated as weakly exogenous for estimation purposes. ${ }^{13}$ Also, by using ten-year averages we aim to cleanse the NFA to GDP ratio of short-run volatility, and effectively condition on the past medium- to long-run trend of a country's international investment position. We considered a number of other specifications of $\tilde{z}_{i t}$ as well, including cumulative current account balances and changes in asset and liability valuation (all scaled by GDP). Employing the NFA to GDP ratio as the conditioning variable proved to be most effective.

For MG estimation of our model, we specify $\alpha_{i}\left(\tilde{z}_{i t}\right)=\alpha_{i}$ and $\boldsymbol{\theta}_{i}\left(\tilde{z}_{i t}\right)=\boldsymbol{\theta}_{i}$. For PMG estimation, we specify $\alpha_{i}\left(\tilde{z}_{i t}\right)=\alpha_{i}$ and $\boldsymbol{\theta}_{i}\left(\tilde{z}_{i t}\right)=\boldsymbol{\theta}$. For CPMG estimation, we specify $\boldsymbol{\theta}_{i}\left(\tilde{z}_{i t}\right)=\boldsymbol{\theta}\left(\tilde{z}_{i t}\right)$, with $\alpha_{i}\left(\tilde{z}_{i t}\right)$ and $\boldsymbol{\theta}\left(\tilde{z}_{i t}\right)$ modelled as first- and third-order Chebyshev polynomials, respectively. For SKMG estimation, we use a Gaussian kernel combined with homogeneous coefficient first-order Chebyshev polynomials to model the state dependence of $\alpha_{i}$ and $\boldsymbol{\theta}_{i}$. Lag orders are selected on the basis of both the Akaike Information Criterion (AIC) and the Schwarz Bayesian Criterion (SBC).

\subsection{Empirical Results}

\subsubsection{Testing the Model Specification}

We begin by examining the stationarity properties of the various variables entering our exchange rate model (27). For this model to be well specified, the model variables should be either integrated of order zero or one, I(0) or I(1), and the long-run levels relation between the model variables should be $\mathrm{I}(0)$. To test for the order of integration of nominal effective exchange rates, $e$, domestic prices, $p$, and weighted foreign prices, $p^{*}$, we employ the panel unit root test of Pesaran (2007). ${ }^{14}$ The results in Table 1(a) provide strong evidence that $p$ and $p^{*}$ are $\mathrm{I}(1)$ variables. Somewhat surprisingly, the evidence in favor of $e$ to be $\mathrm{I}(1)$ is less compelling. However, as the unit root test statistic for the level of $e$ was insignificant at the one percent level when the cross-sectional augmentation term was dropped, we proceed with the consensus view in the literature that $e$ is best modelled as I(1). We invoke the test statistic proposed by Westerlund (2005) to test for (conditional) panel cointegration between $e, p$ and $p^{*}$, that is, the existence of an $\mathrm{I}(0)$ relation between $e, p$ and $p^{*}$ depending on our conditioning variable $\tilde{z}$; Appendix D provides details on the test statistic and its applicability for our panel modelling approach. Table $1(\mathrm{~b})$ provides evidence that $e-\theta_{1}(\tilde{z}) p-\theta_{2}(\tilde{z}) p^{*}$ is $\mathrm{I}(0)$. The results in Table 1 (b) are based on a third-order Chebyshev polynomial specification

\footnotetext{
${ }^{13}$ For the beginning of the sample, however, to avoid losing numerous observations the moving average is computed using a fixed window involving the observations in the first ten years of the sample, that is, we compute $\tilde{z}_{i t}=1 / 10 \cdot \sum_{s=\max (t-10,1)}^{\max (t-1,10)} z_{i s}, t=1,2, \ldots, T_{i}$.

${ }^{14}$ This panel unit root test inter alia allows for two features of the data that we stress in this paper: country-specific short-run dynamics and cross-country correlation of the error terms.
} 
of $\boldsymbol{\theta}(\tilde{z})$, but also hold when the polynomial order is reduced to zero or one. Overall, Table 1 provides strong support for the exchange rate model (27) being an appropriate model formulation concerning (non-)stationarity of the model variables.

\subsubsection{Estimation Results for the Full Sample}

We can thus turn to estimation results for the exchange rate model (27). Table 2 reports the long-run coefficients on $p$ and $p^{*}$ in the long-run relation between effective nominal exchange rates, domestic prices and weighted foreign prices, as well as the speed of adjustment to this long-run relation under the different estimation procedures we consider. The first two columns report MG and PMG estimation results, whereas the third and fourth columns show the average estimates across all values of the conditioning variable, the NFA to GDP ratio, obtained under CPMG and SKMG. In contrast to the MG estimates that do not involve any form of pooling, the estimates of both long-run parameters based on all other estimation procedures are highly significant. It may be worth pointing out explicitly that the standard errors under CPMG and even under SKMG are smaller yet than those under PMG, providing some support for the CPMG and SKMG procedures we are proposing in this paper to be effective procedures for the number of observations available in many cross-country macroeconomic panels. ${ }^{15}$ Note that at least from a statistical perspective unconditional PPP, that is $\theta_{1}=1$ and $\theta_{2}=-1$ across all values of the NFA to GDP ratio, is clearly rejected under the PMG, CPMG and SKMG procedures. All point estimates of the longrun parameters for $\theta_{1}$ fall in the interval [0.55, 0.74], and those for $\theta_{2}$ fall into the interval $[-0.88,-0.68]$ and suggest a stronger long-run reaction of effective nominal exchange rates to weighted foreign prices as compared to domestic prices. It is quite remarkable that the estimates of the speed of adjustment coefficients all suggest rather fast adjustment to the long-run relation, in particular implying half lives between one and two years, much faster than what has typically been found in the literature and removing most of the stickiness puzzle that the previous literature on PPP (see, for example, Rogoff, 1996) argued to be present.

While the average parameter estimates for CPMG and SKMG across all values of the NFA to GDP ratio are qualitatively similar to those obtained under the PMG approach, the idea underlying our CPMG and SKMG approaches is, of course, to report on the variation of the speed of adjustment and long-run coefficients across different values of the NFA to GDP ratio. Figures 3 to 18 pick up on this point. Figures 3 and 5 convey that for our full sample of 71 countries there appears to be a strong dependence of the two long-run coefficients for domestic and weighted foreign prices on a country's international investment position

\footnotetext{
${ }^{15}$ While a systematic investigation of the finite sample properties of the CPMG and SKMG estimators proposed in this paper will be valuable, this is beyond the scope of the current paper.
} 
as reflected by the NFA to GDP ratio. In particular, in environments of limited negative NFA to GDP ratios we find rather strong evidence that foreign exchange markets appear to view the PPP relation as a strong anchor for the pricing of currencies. Under a limited negative NFA to GDP ratio, the long-run coefficients on domestic and weighted foreign prices are economically and partially even statistically insignificantly different from one and minus one, respectively. The boundaries of this limited negative NFA to GDP ratio are rather similar under the CPMG and SKMG procedures: about minus two thirds to minus one third under the CPMG approach, and about minus three quarters to minus one third under the SKMG approach. For other states of the international investment position, the long-run relation bears limited, little or even no resemblance with what PPP would suggest. When the NFA to GDP ratio is smaller than minus one, the SKMG approach suggests long-run elasticities of the effective nominal exchange rate with respect to domestic and weighted foreign prices of less than one half in absolute value. The elasticities do not drop as strongly under the CPMG approach; however, the CPMG standard error bands widen sizeably for NFA to GDP ratios of less than minus one and a half, reflecting the very limited number of such values in our sample. It thus appears that under negative NFA to GDP ratios that suggest a high degree of external imbalance, foreign exchange markets abandon PPP as a medium- to long-run anchor. PPP also appears to have limited to no importance when the NFA to GDP ratio is approaching zero and even when there is a limited positive NFA to GDP ratio. The latter result may, however, also reflect too few observations in our sample for which the NFA to GDP ratio exceeds plus one fifth.

Figures 4 and 6 report on the speed of adjustment coefficients for our full sample under the CPMG and SKMG approaches. Under the SKMG approach the speed of adjustment coefficients vary very little with the NFA to GDP ratio. While Figure 4 suggests that under the CPMG approach the speed of adjustment coefficients vary considerably more across NFA to GDP ratios than under the SKMG approach, such variation occurs primarily outside the interval from minus one to zero: For NFA to GDP ratios less than minus one, the speed of adjustment coefficient increases rapidly, whereas it tends towards zero for NFA to GDP ratios larger than plus one fifth. Once more, however, it appears prudent not to put strong emphasis on results obtained for values of the NFA to GDP ratio larger than plus one fifth, given the limited number of observations in our sample involving such NFA to GDP ratios. Overall, Figures 3 to 6 provide rather strong evidence that the NFA to GDP ratio significantly influences medium- to long-run exchange rate dynamics, but has limited, if any, effect on short-run dynamics. Medium- to long-run exchange rate dynamics under limited negative NFA to GDP ratios seem well characterized by PPP. 


\subsubsection{Robustness Analysis Using Sample Splits}

In addition to the international investment position of a country, it is likely that its mediumto long-run exchange rate dynamics are also influenced by other features of its macroeconomic and financial environment such as its income level, its exchange rate regime or its degree of price variability. ${ }^{16}$ One reason that the income level may matter is that if a country's NFA to GDP ratio was on average negatively related to its income level (and we will document evidence for this to be the case), then it would seem reasonable to conjecture that the threshold as to when a negative NFA to GDP ratio is judged by foreign exchange market participants to indicate imbalance depends in absolute value negatively on the level of income. Depending on the exchange rate regime, imbalances of real exchange rates may be judged to be more or less sustainable, resulting in differing degrees of conformity of the exchange rate with price fundamentals. Regarding price variability, a relatively high degree of price variability will ceteris paribus lead to a larger number of situations where imbalances will be sufficiently pronounced to require correction and thus might result in PPP equilibrium being a more relevant description of medium- to long-run exchange rate dynamics.

As a first analysis to what extent our results regarding the role of a country's international investment position for medium- to long-run exchange rate dynamics are sensitive to also modelling other factors of the macroeconomic and financial environment, we therefore include income level, exchange rate regime and price variability information in our analysis to disentangle the impact of these factors from that of the international investment position. It would clearly be appealing to allow for multi-factor conditioning through a CPMG or SKMG model that conditioned on a vector of state variables. However, in the setup of our panel model of Section 3 this could easily result in a loss of parameter parsimony. How to best reconcile parsimony with the CPMG and SKMG approaches is left for future research. In this paper, we instead confine ourselves to documenting the variation of the long-run elasticity of the effective nominal exchange rate with respect to domestic and weighted foreign prices across differing NFA to GDP ratios for three sample splits: (i) industrial and emerging market countries vs. developing countries, (ii) sticky exchange rate regimes vs. floating exchange rate regimes and (iii) countries with a relatively high degree of price variability vs. countries with a relatively low degree of price variability.

Beginning with the income level based split, we split our sample of 71 countries into three groups, following the World Bank's income-based classification of countries. ${ }^{17}$ In particular, we label countries categorized by the World Bank as "high-income OECD countries" as

\footnotetext{
${ }^{16}$ Previous literature on the PPP hypothesis has argued that the stationarity properties of real exchange rates may depend on such factors (see, for example, Cheung and Lai, 2000, or Binder, Pesaran and Sharma, 2004).

${ }^{17}$ See http://go.worldbank.org/D7SNOB8Yuo, accessed on April 26, 2006.
} 
"industrial countries", label the World Bank's "high-income non-OECD countries" as well as "upper middle-income countries" as "emerging markets" and finally label the World Bank's "lower middle-income countries" as well as "low-income countries" as "developing countries". We find that for this sample split the average NFA to GDP ratio is equal to -0.175 for the industrial and emerging market countries, and is equal to -0.576 for the developing countries. Figures 7 to 10 suggest that the relation between medium- to long-run exchange rate pricing and a country's international investment position that we found for the full sample of countries is also present both for our industrial and emerging market countries sample as well as our developing countries sample, with some quantitative qualifications. For the industrial and emerging market countries sample the range of limited negative NFA to GDP ratios for which we observe PPP-type medium- to long-run relations is associated with smaller investment position imbalances than for the sample comprising all countries, extending now over an interval from about minus one half to minus one tenth. Furthermore, both under CPMG and SKMG the absolute values of the long-run elasticities of the effective nominal exchange rate with respect to domestic and weighted foreign prices fall relative to their PPP values by rather small amounts only for values of the NFA to GDP ratio that are less than minus one half. At least under the CPMG approach these elasticities fall a good bit more strongly for zero and positive values of the NFA to GDP ratio. Overall, it appears that - as we had conjectured - foreign exchange markets for industrial and emerging market countries view PPP as a relevant anchor for smaller (negative) imbalances of the international investment position than for developing countries. For the latter, the range of NFA to GDP ratios under which we observe PPP-type medium- and long-run relations appears to be both under CPMG and SKMG about minus two thirds to minus one third.

To consider the role of exchange rate regimes for our results, we employ a data set on the de facto classification of exchange rate flexibility assembled by Levy-Yeyati and Sturzenegger (2005). Their data set contains an annual five-way categorization of the exchange rate regimes of inter alia all the 71 countries considered in our analysis as "flexible", "dirty float", "inconclusive", "crawling peg" and "fixed". We recode these five categories from a value of one for a "flexible" exchange rate regime to a value of five for a "fixed" exchange rate regime. ${ }^{18}$ Our sample split then constructs two groups of countries: The first group consists of countries for which the exchange rate classification code over our sample period is on average at most equal to three, and the second group features all countries with an exchange rate classification code being on average larger than three over our sample period. Figures 11 to 14 report on our exchange rate regime based sample splits. Inspection of these figures reveals that the curvatures of the functions depicting the long-run coefficients in dependence of the NFA to GDP ratio actually differ more sizeably across the CPMG

\footnotetext{
${ }^{18}$ The Levy-Yeyati and Sturzenegger (2005) data set spans the period 1974 to 2004; we assume that all exchange rates were "fixed" over the period 1970 to 1973.
} 
and SKMG estimation approaches than across the two sub-samples of sticky and floating exchange rate regimes. This suggests that our international investment position conditioning is separate from any influence of exchange rate regimes on medium- to long-run exchange rate dynamics. Figures 11 to 14 indeed suggest the even stronger interpretation that exchange rate regimes matter little for medium- to long-run pricing in foreign exchange markets, at least for the sample of countries we are considering. ${ }^{19}$

To consider the impact of a country's degree of price variability on our results, we split our sample into one group of countries for which the average rate of inflation over our sample period exceeded eight percent (we label countries in this group as those exhibiting a "high degree of price variability") as well as a second group of countries for which the average rate of inflation over our sample period was eight percent or lower (we label countries in this group as those exhibiting a "low degree of price variability"). Figures 15 to 18 report on our sample split between countries with a high degree of price variability (relatively high rates of inflation) vs. countries with a low degree of price variability (relatively low rates of inflation). The figures suggest that the magnitudes of international investment position imbalances under which foreign exchange markets in the medium to long run price currencies in line with PPP are sensitive to the degree of price variability. The CPMG estimates in particular convey that limited negative NFA to GDP ratios for countries with a high degree of price variability are centered around minus two thirds to minus one half, but for countries with a low degree of price variability are in absolute value sizeably larger, namely are centered around minus one approximately. In other words, under relatively high degrees of price stability, foreign exchange markets return to PPP fundamentals only under higher degrees of external imbalance than in environments of relatively low degrees of price stability. This is consistent with the intuition that arbitrage opportunities tend to be more likely present in environments of low degrees of price stability. ${ }^{20}$

\footnotetext{
${ }^{19}$ It should be kept in mind, of course, that we work with effective exchange rates spanning a broad range of countries, whereas the Levy-Yeyati and Sturzenegger (2005) classification concerns fluctuations of a currency relative to one selected currency (often the U.S. Dollar, the Pound Sterling, the Deutsche Mark or the French Franc) only.

${ }^{20}$ The rank correlation coefficient between our income level based sample split and the price variability based sample split (coding for the income level based sample split an industrial/emerging market country as 1 and a developing country as 2; and for the price variability based sample split a low price variability country as 1 and a high price variability country as 2) is 0.317. A developing country is more likely to also be a high price variability country. As for a developing country the range of NFA to GDP ratios that we categorize as limited negative is in absolute values larger than for an industrial/emerging market country, but for a country with high degree of price variability the range of NFA to GDP ratios that we categorize as limited negative is in absolute values smaller than for countries with a low degree of price variability, the results for income level and price variability based sample splits are indeed distinct results.
} 


\subsubsection{Exchange Rate Projections: The Effects of Changes in the NFA to GDP Position on Nominal and Real Exchange Rates}

Finally, let us turn to discussing the potential implications of our in-sample estimation results for out-of-sample exchange rate developments. In particular, we wish to examine how under different scenarios for a country's international investment position as measured by the NFA to GDP ratio its nominal and real exchange rates would evolve. Under a plausible scenario regarding the rate of growth of domestic and weighted foreign prices, will a reversion to PPPbased exchange rate pricing per se help with removing the imbalance in the international investment position by implying a real depreciation of the domestic currency? If so, what will be the magnitude of this depreciation?

To compute our out-of-sample projections, we start from the long-run relation between nominal exchange rates, domestic prices and weighted foreign prices that is implied by our model (27), is conditional on the state of the NFA to GDP ratio, and is affected also by the long-run impact of the common factors:

$$
\begin{aligned}
e_{i t}^{L R} & =\hat{\theta}_{1}\left(\tilde{z}_{i}\right) p_{i t}+\hat{\theta}_{2}\left(\tilde{z}_{i}\right) p_{i t}^{*}-\left[\hat{\alpha}_{i}\left(\tilde{z}_{i}\right)\right]^{-1}\left[\hat{\mu}_{i}+\hat{\eta}_{i} \bar{e}_{t}+\hat{\zeta}_{i}\left(\bar{p}_{t}-\bar{p}_{t}^{*}\right)\right] \\
& =\hat{\theta}_{1}\left(\tilde{z}_{i}\right) p_{i t}+\hat{\theta}_{2}\left(\tilde{z}_{i}\right) p_{i t}^{*}-\left[\hat{\alpha}_{i}\left(\tilde{z}_{i}\right)\right]^{-1}\left[\hat{\mu}_{i}+\hat{\boldsymbol{\chi}}_{i}^{\prime} \boldsymbol{g}_{t}\right] .
\end{aligned}
$$

For purposes of our projections we use the relative PPP interpretation of (28) in terms of changes of the variables involved:

$$
\begin{aligned}
\Delta e_{i t}^{L R} & =\hat{\theta}_{1}\left(\tilde{z}_{i}\right) \Delta p_{i t}+\hat{\theta}_{2}\left(\tilde{z}_{i}\right) \Delta p_{i t}^{*}-\left[\hat{\alpha}_{i}\left(\tilde{z}_{i}\right)\right]^{-1}\left[\hat{\eta}_{i} \Delta \bar{e}_{t}+\hat{\zeta}_{i} \Delta\left(\bar{p}_{t}-\bar{p}_{t}^{*}\right)\right] \\
& =\hat{\theta}_{1}\left(\tilde{z}_{i}\right) \Delta p_{i t}+\hat{\theta}_{2}\left(\tilde{z}_{i}\right) \Delta p_{i t}^{*}-\left[\hat{\alpha}_{i}\left(\tilde{z}_{i}\right)\right]^{-1} \hat{\boldsymbol{\chi}}_{i}^{\prime} \Delta \boldsymbol{g}_{t} .
\end{aligned}
$$

To obtain the ten-year, out-of-sample projections of nominal and real effective exchange rates based on the relative long-run relation (29), we compute

$$
\begin{aligned}
\operatorname{Proj}_{2004}^{(k)}\left(\Delta e_{i, 2004+s}^{L R}\right) & =\hat{\theta}_{1}\left(\tilde{z}_{i}^{(k)}\right) \operatorname{Proj}_{2004}\left(\Delta p_{i, 2004+s}\right)+\hat{\theta}_{2}\left(\tilde{z}_{i}^{(k)}\right) \operatorname{Proj}_{2004}\left(\Delta p_{i, 2004+s}^{*}\right) \\
& -\left[\hat{\alpha}_{i}\left(\tilde{z}_{i}^{(k)}\right)\right]^{-1} \hat{\boldsymbol{\chi}}_{i}^{\prime} \operatorname{Proj}_{2004}\left(\Delta \boldsymbol{g}_{2004+s}\right), \quad s=1,2, \ldots, 10, \quad k=0,1,2,
\end{aligned}
$$

where all counterfactual projections $(k=0,1,2)$ postulate that domestic prices, weighted foreign prices and common factors change over the projection period at a rate that equals their annualized rate of change over the previous ten-year time period, that is, for $s=$ $1,2, \ldots, 10$,

$$
\begin{aligned}
\operatorname{Proj}_{2004}\left(\Delta p_{i, 2004+s}\right) & =\frac{1}{10}\left[\operatorname{Proj}_{2004}\left(p_{i, 2004+s-1}\right)-\operatorname{Proj}_{2004}\left(p_{i, 2004+s-11}\right)\right], \\
\operatorname{Proj}_{2004}\left(\Delta p_{i, 2004+s}^{*}\right) & =\frac{1}{10}\left[\operatorname{Proj}_{2004}\left(p_{i, 2004+s-1}^{*}\right)-\operatorname{Proj}_{2004}\left(p_{i, 2004+s-11}^{*}\right)\right], \\
\operatorname{Proj}_{2004}\left(\Delta \boldsymbol{g}_{2004+s}\right) & =\frac{1}{10}\left[\operatorname{Proj}_{2004}\left(\boldsymbol{g}_{2004+s-1}\right)-\operatorname{Proj}_{2004}\left(\boldsymbol{g}_{2004+s-11}\right)\right],
\end{aligned}
$$


with $\operatorname{Proj}_{2004}\left(p_{i, 2004+\tau}\right)=p_{i, 2004+\tau}, \operatorname{Proj}_{2004}\left(p_{i, 2004+\tau}^{*}\right)=p_{i, 2004+\tau}^{*}$ and $\operatorname{Proj}_{2004}\left(\boldsymbol{g}_{2004+\tau}\right)=$ $\boldsymbol{g}_{2004+\tau}$ for $\tau \leq 0$.

The projections differ with respect to the assumption about the international investment position. For the baseline projection, $\operatorname{Proj}_{2004}^{(0)}$, we assume that the NFA to GDP position throughout the projection period remains at its 2004 smoothed level, that is, we assume

$$
\tilde{z}_{i}^{(0)}=\tilde{z}_{i, 2004}
$$

For the second projection, $\operatorname{Proj}_{2004}^{(1)}$, we assume that throughout the projection period the NFA to GDP ratio is equal to a level obtained from adding to the 2004 level of the smoothed NFA to GDP ratio the total change of the smoothed NFA to GDP ratio that would have resulted had the smoothed NFA to GDP ratio grown for ten years at a rate equal to its rate of change over the previous five-year time period, that is, we compute

$$
\tilde{z}_{i}^{(1)}=\tilde{z}_{i, 2004}+\operatorname{Proj}_{2004}\left(\Delta \tilde{z}_{i}\right)
$$

where

$$
\operatorname{Proj}_{2004}\left(\Delta \tilde{z}_{i}\right)=\sum_{s=1}^{10} \frac{1}{5}\left[\operatorname{Proj}_{2004}\left(\tilde{z}_{i, 2004+s-1}\right)-\operatorname{Proj}_{2004}\left(\tilde{z}_{i, 2004+s-6}\right)\right],
$$

with $\operatorname{Proj}_{2004}\left(\tilde{z}_{i, 2004+\tau}\right)=\tilde{z}_{i, 2004+\tau}$ for $\tau \leq 0$.

For the third projection, we assume yet more sizeable changes in the NFA to GDP ratio and compute

$$
\tilde{z}_{i}^{(2)}=\tilde{z}_{i, 2004}+2 \cdot \operatorname{Proj}_{2004}\left(\Delta \tilde{z}_{i}\right)
$$

with $\operatorname{Proj}_{2004}\left(\Delta \tilde{z}_{i}\right)$ given by (36). That is, for the third projection we postulate for each country double the change of the NFA to GDP ratio that we postulated for our second projection.

Figures 19 to 27 report on these projections for nine currencies in our sample, three each from the industrial, emerging market and developing countries sub-samples: the U.S. Dollar, the British Pound Sterling, the Spanish Peseta, the Mexican Peso, the Turkish Lira, the Uruguayan Peso, the Indian Rupee, the Paraguayan Guarani and the Thai Baht. The currencies were selected to illustrate settings for which we can compare exchange rate change trajectories involving NFA to GDP positions that vary within or close to the range of what we had called limited negative NFA to GDP positions.

In each of the Figures 19 to 27, the table in sub-panel (a) summarizes the effects of the three alternative projection states of the NFA to GDP ratio on the parameters of interest, namely the long-run coefficient on domestic prices, $\theta_{1}$, the long-run coefficient on weighted foreign prices, $\theta_{2}$, and the speed of adjustment coefficient, $\alpha$. Sub-panels (b) and (c) depict the actual (left to the 2004 vertical line) and projected (right to the 2004 vertical line) 
values of domestic and weighted foreign prices as well as the common factor effects, $\hat{\boldsymbol{\chi}}_{i}^{\prime} \boldsymbol{g}_{t}$. Sub-panels (d) and (e) show the projections of the resultant changes in effective nominal and real exchange rates, conditional on the three alternative projection states of the NFA to GDP ratio. Here, the (+)-symbol (blue) line represents the baseline projection keeping the NFA to GDP ratio at its 2004 smoothed level throughout the projection period (that is, the projection in (30) under $k=0$ ). For the United States, for example, this projection reveals that due to (i) a higher weighted rate of inflation abroad than domestically, also taking into account in absolute value larger long-run elasticities of exchange rates with respect to weighted foreign than domestic prices, and (ii) the common factor effect we project the U.S. Dollar to appreciate throughout the projection period (with some levelling off of the magnitude of the appreciation in the second half of the projection period). The $(\times)$-symbol (red) lines in Figures $19(\mathrm{~d})$ and (e) depict the projection in (30) under $k=1$, and the (o)-symbol (green) lines in Figures 19(d) and (e) depict the projection in (30) under $k=2$, that is, these are the projections for which for the United States the NFA to GDP ratio throughout the projection period is assumed to be -0.48 (for $k=1$ ) and -0.69 (for $k=2$ ) rather than -0.28 as under the baseline projection $(k=0)$. We can see that under NFA to GDP ratios of -0.48 and -0.69 the long-run coefficients on domestic and weighted foreign prices are closer to plus/minus one, reflecting shift of the NFA to GDP ratio to what for the full country sample we had categorized as a limited negative value. The absolute changes in the two long-run coefficients, $\theta_{1}$ and $\theta_{2}$, are quite similar in magnitude, both as we move from $k=0$ to $k=1$ and as we move from $k=1$ to $k=2$. It should also be noted that for the United States, the speed of adjustment coefficient shows more variation with changes in the NFA to GDP position than what occurs for our full sample; the half-life of shocks to the long-run relation is around eight months at an NFA to GDP ratio of -0.48 and around four months at an NFA to GDP ratio of -0.69 .

As inspection of Equation (30) reveals, the overall depreciating exchange rate effects of a deterioration of the NFA to GDP ratio for the United States are thus due to the appreciation inducing common factor effect having less impact on the long-run relation due to faster speed of adjustment to the medium- to long-run relation (in light of higher rates of inflation abroad than domestically, the isolated effect of moving closer to PPP pricing for the United States in contrast is a slight appreciation). Table 3 lists the exchange rate effects. For the United States, an NFA to GDP position of -0.48 rather than -0.28 under the projected price and common factor effect changes would imply that the overall appreciation of the U.S. Dollar effective exchange rate would be $0.4 \%$ smaller over the first year of the projection period, and $2.46 \%$ smaller when cumulated over the entire ten year projection period. These effects increase to $0.55 \%$ and $3.32 \%$, respectively, when the NFA to GDP position is equal to -0.69 rather than -0.28 . We thus find that a growing deficit in the international investment 
position of the United States ceteris paribus leads in nominal and real terms to a (small) depreciation of the U.S. Dollar. ${ }^{21}$

Figures 19 to 27 show that for seven out of the nine countries considered in our projections, the NFA to GDP position under the $k=1$ and $k=2$ projections reflects a larger deficit in the international investment position than under $k=0$. Deterioration of the NFA to GDP position in our projections beyond the United States applies to the United Kingdom, Spain, Mexico, Turkey, Uruguay and Paraguay. Our projections involve improvements of the NFA to GDP position for India and Thailand.

Of the seven countries for which our projections feature a decreasing NFA to GDP position, the deterioration for five countries (beyond the United States, these are Mexico, Turkey, Uruguay and Paraguay), ceteris paribus has a depreciating effect on the effective nominal and real exchange rates. The effects are particularly pronounced for the Turkish Lira and the Uruguayan Peso, in real terms under a decrease of the NFA to GDP ratio from -0.22 to -0.35 for Turkey amounting to $27.85 \%$ when cumulated over ten years, and to $22.54 \%$ cumulated over the ten year horizon under a decrease of the NFA to GDP ratio from -0.23 to -0.6 for Uruguay. (Table 4 puts all effects in relation to the size and direction of change of the NFA to GDP position.) For Turkey, this result reflects that as the long-run coefficients $\theta_{1}$ and $\theta_{2}$ under the decrease of the NFA to GDP ratio move closer to their PPP values of plus one and minus one, this in net terms causes a sizeable depreciating effect due to domestic inflation being significantly higher than inflation abroad. For Uruguay, the depreciating exchange rate effect of a deteriorating NFA to GDP position reflects both the diminution of the appreciation inducing common factor effect (due to faster reversal to the long-run relation) and the fact that higher inflation domestically than abroad has a strengthened depreciating effect when exchange rate pricing is more closely in line with PPP. In contrast, for two countries, the United Kingdom and Spain, our projection results indicate a very small appreciating effect that ceteris paribus is set off by a worsening of the NFA to GDP position. This is due to the fact that there is no notable depreciating effect from the common factor effect for these two countries, and that exchange rate pricing more closely in line with PPP due to slightly higher inflation abroad than domestically has a small appreciating effect on the Pound Sterling and the Spanish Peseta.

For the two countries for which our projections involve improvements of the NFA to GDP position, India and Thailand, the projections indicate a relative appreciation of the Indian Rupee and a relative depreciation of the Thai Baht. For the Rupee, this occurs as exchange rate pricing less closely in line with PPP implies less weight for domestic inflation (that for India is projected to be higher than inflation abroad). In the case of the Baht, despite

\footnotetext{
${ }^{21}$ Note, of course, that our projections do not model the feedback from exchange rate depreciation onto the current account and onto the international investment position.
} 
improvement of the NFA to GDP position under our projections, the Baht is projected to depreciate as the depreciation inducing common factor effect gains more weight due to the higher persistence of deviations from the long-run relation under a more balanced international investment position.

Overall, the results reflect that our conditioning of the interrelation between nominal exchange rates, domestic prices and weighted foreign prices on the NFA to GDP position implies that the effect of a change in the NFA to GDP position on the exchange rate depends on the rate of inflation. In particular, considering environments in which deterioration of a country's NFA to GDP position leads to an increased relevance of PPP based exchange rate pricing, the depreciating effect of this deterioration depends on the magnitude of domestic inflation relative to the magnitude of inflation abroad: If inflation is higher domestically than abroad, then for all countries we have studied a deterioration of the NFA to GDP position per se caused exchange rate depreciation. If inflation is higher abroad than domestically, however, then for all countries we have studied the direction of change of the exchange rate implied by a deterioration of the NFA to GDP position critically depended on the degree to which the common factor effect would, due to changes in the persistence of deviations from the long-run relation, matter more or matter less.

\section{Conclusion}

In this paper we have revisited medium- to long-run exchange rate determination, focusing on the role of international investment positions. To do so, we have developed a new econometric framework accounting for conditional long-run homogeneity in heterogeneous dynamic panel data models. In particular, in our model the long-run relationship between exchange rates and domestic as well as weighted foreign prices has been specified as a function of a country's international investment position as measured by smoothed lagged values of the NFA to GDP position. We have found rather strong support for PPP in environments of limited negative net foreign asset to GDP positions, but not outside such environments. We also added evidence that the conditioning of PPP on a country's international investment position remains important when allowing for other features of the macroeconomic environment, such as the income level, the exchange rate regime and the degree of price variability. Using counterfactual projections, we investigated the implications of these results for the relation between changes in a country's NFA to GDP position and its effective nominal and real exchange rate. We found that this relation varies widely across countries, depending on inflation dynamics and exposure to global shocks. A deterioration of a country's NFA to GDP position tends to have the largest depreciating exchange rate effects (both nominal and real) when domestic inflation significantly exceeds inflation abroad. 
Our future research will in particular address two issues: (i) the extension of CPMG and SKMG models to a parsimonious multivariate conditioning framework, and (ii) the extension of at least part of our database to bilateral measurement of international capital flows, allowing to address issues of links between the sources and destinations of capital flows as well as their effects on stocks of international investment positions on the one hand and macroeconomic and financial market outcomes on the other hand. 


\section{Tables and Figures}

Table 1: Stationarity Properties for 71 Countries, 1970 to 2004

\begin{tabular}{lcc}
\hline \hline & (a) Panel Unit-Root Test & \\
& Level & First Difference \\
\cline { 2 - 2 }$e$ & $\mathbf{- 2 . 9 8 0 5}$ & $-\mathbf{3 . 3 1 1 3}$ \\
$p$ & -1.9815 & $-\mathbf{2 . 7 2 7 1}$ \\
$p^{*}$ & -2.3187 & $-\mathbf{3 . 3 0 1 4}$ \\
\hline
\end{tabular}

(b) Panel Cointegration Test

\begin{tabular}{c}
$\frac{V R_{P}}{-4.9449}$ \\
\hline \hline
\end{tabular}

Notes: The panel unit-root test (part (a)) is computed according to Pesaran (2007) and has a non-standard distribution under the null hypothesis of a unit root in the time series under consideration for all countries. Under the alternative hypothesis, the variable is $\mathrm{I}(0)$ for a non-vanishing share of countries. Levels of the variables are modelled with a constant and a linear time trend, whereas the specifications for first differences of the variables include a constant only. The critical value at the $5 \%(1 \%)$ significance level for the level of a variable is $-2.58(-2.69)$ and $-2.08(-2.19)$ for the first difference of a variable. The panel cointegration test statistic (part (b)) is distributed standard Normal under the null of no cointegration. $V R_{P}$ refers to the Panel Variance Ratio Statistic in Westerlund (2005), which has the alternative hypothesis that cointegration prevails for all countries. The test statistic was computed using Chebyshev polynomials of order three for the estimation of conditionally homogeneous long-run coefficients. The lag orders in both parts of the table were selected according to the Akaike Information Criterion based on a maximum lag length of 2 , but the results are robust to other choices, as well as to lag selection on the basis of other information criteria such as the Schwarz Bayesian Criterion. Figures in bold face denote significance at the $5 \%$ level.

Table 2: Speed of Adjustment and Long-Run Coefficients (Averages)

\begin{tabular}{l|cc|cc}
\hline \hline & MG & PMG & CPMG & SKMG \\
\hline$\alpha$ & $-\mathbf{0 . 4 3 2 5}$ & $-\mathbf{0 . 3 1 1 0}$ & $-\mathbf{0 . 3 5 3 3}$ & $-\mathbf{0 . 3 0 7 4}$ \\
& $(0.0339)$ & $(0.0244)$ & $(0.0021)$ & $(0.0000)$ \\
$\theta_{1}$ & 0.1715 & $\mathbf{0 . 5 5 1 0}$ & $\mathbf{0 . 7 3 7 4}$ & $\mathbf{0 . 6 3 0 4}$ \\
& $(0.2238)$ & $(0.0896)$ & $(0.0314)$ & $(0.0285)$ \\
$\theta_{2}$ & $-\mathbf{1 . 0 8 8 2}$ & $-\mathbf{0 . 8 0 9 7}$ & $-\mathbf{0 . 8 7 3 6}$ & $-\mathbf{0 . 6 8 9 7}$ \\
& $(0.2131)$ & $(0.0779)$ & $(0.0322)$ & $(0.0310)$ \\
\hline \hline
\end{tabular}

Notes: Cross-country averages of the speed of adjustment coefficient $\alpha$ and the long-run coefficients on the domestic $\left(\theta_{1}\right)$ and weighted foreign $\left(\theta_{2}\right)$ prices. PPP would suggest that $\alpha<0, \theta_{1}=1$, and $\theta_{2}=-1$. Under CPMG and SKMG, country-specific coefficients are evaluated at the mean of the conditioning variable $\tilde{z}_{i t}$. The lag length is selected according to Akaike Information Criterion with a maximum lag of 2. Standard errors are given in parentheses below the coefficients; figures in bold face denote significance at the $5 \%$ level. 
Table 3: Cumulated Effect of Changes in the International Investment Position

\begin{tabular}{lccc|ccc}
\hline \hline & \multicolumn{3}{c|}{ Scenario 1 } & \multicolumn{3}{c}{ Scenario 2 } \\
& 1 & 5 & 10 & 1 & 5 & 10 \\
\hline U.S. Dollar & 0.40 & 1.28 & 2.46 & 0.55 & 1.71 & 3.32 \\
Pound Sterling & -0.05 & -0.21 & -0.39 & -0.10 & -0.42 & -0.77 \\
Spanish Peseta & 0.00 & -0.47 & -0.87 & 0.01 & -0.98 & -1.84 \\
Mexican Peso & 0.38 & 1.14 & 1.73 & 0.73 & 2.21 & 3.37 \\
Turkish Lira & 1.53 & 7.80 & 14.33 & 2.99 & 15.16 & 27.85 \\
Uruguayan Peso & 2.84 & 7.20 & 13.45 & 4.64 & 12.05 & 22.54 \\
Indian Rupee & -0.01 & -0.54 & -1.01 & -0.04 & -1.08 & -2.01 \\
Paraguayan Guarani & 0.16 & 1.75 & 3.21 & 0.13 & 2.41 & 4.37 \\
Thai Baht & 0.29 & 0.83 & 1.56 & 0.64 & 1.80 & 3.37 \\
\hline \hline
\end{tabular}

Notes: Sum of differences between exchange rate changes in percentage points after one, five and ten years. Scenario 1: Comparison between projections based on $\tilde{z}_{i}^{(1)}$ relative to projections based on $\tilde{z}_{i}^{(0)}$. Scenario 2: Comparison between projections based on $\tilde{z}_{i}^{(2)}$ relative to projections based on $\tilde{z}_{i}^{(0)}$. See Section 5.2.4 for further details.

Table 4: Cumulated Relative Effect of Changes in the International Investment Position

\begin{tabular}{lccc|ccc}
\hline \hline & \multicolumn{3}{c|}{ Scenario 1} & \multicolumn{3}{c}{ Scenario 2 } \\
& 1 & 5 & 10 & 1 & 5 & 10 \\
\hline U.S. Dollar & -1.94 & -6.21 & -11.95 & -1.34 & -4.17 & -8.08 \\
Pound Sterling & 0.34 & 1.45 & 2.67 & 0.33 & 1.43 & 2.65 \\
Spanish Peseta & -0.01 & 6.27 & 11.64 & -0.05 & 6.60 & 12.30 \\
Mexican Peso & -3.81 & -11.29 & -17.08 & -3.63 & -10.93 & -16.69 \\
Turkish Lira & -23.59 & -120.2 & -220.8 & -23.01 & -116.8 & -214.5 \\
Uruguayan Peso & -15.15 & -38.38 & -71.69 & -12.36 & -32.11 & -60.06 \\
Indian Rupee & -0.28 & -13.17 & -24.59 & -0.45 & -13.09 & -24.37 \\
Paraguayan Guarani & -0.56 & -6.23 & -11.40 & -0.23 & -4.28 & -7.75 \\
Thai Baht & 9.08 & 25.68 & 48.01 & 9.81 & 27.73 & 51.83 \\
\hline \hline
\end{tabular}

Notes: Sum of differences between exchange rate changes in percentage points after one, five and ten years relative to the size and direction of the change in the NFA to GDP position. Scenarios 1 and 2 are defined as in Table 3. 
Figure 1: Gross Asset and Liability Stocks as a Ratio to GDP, 1970 to 2004

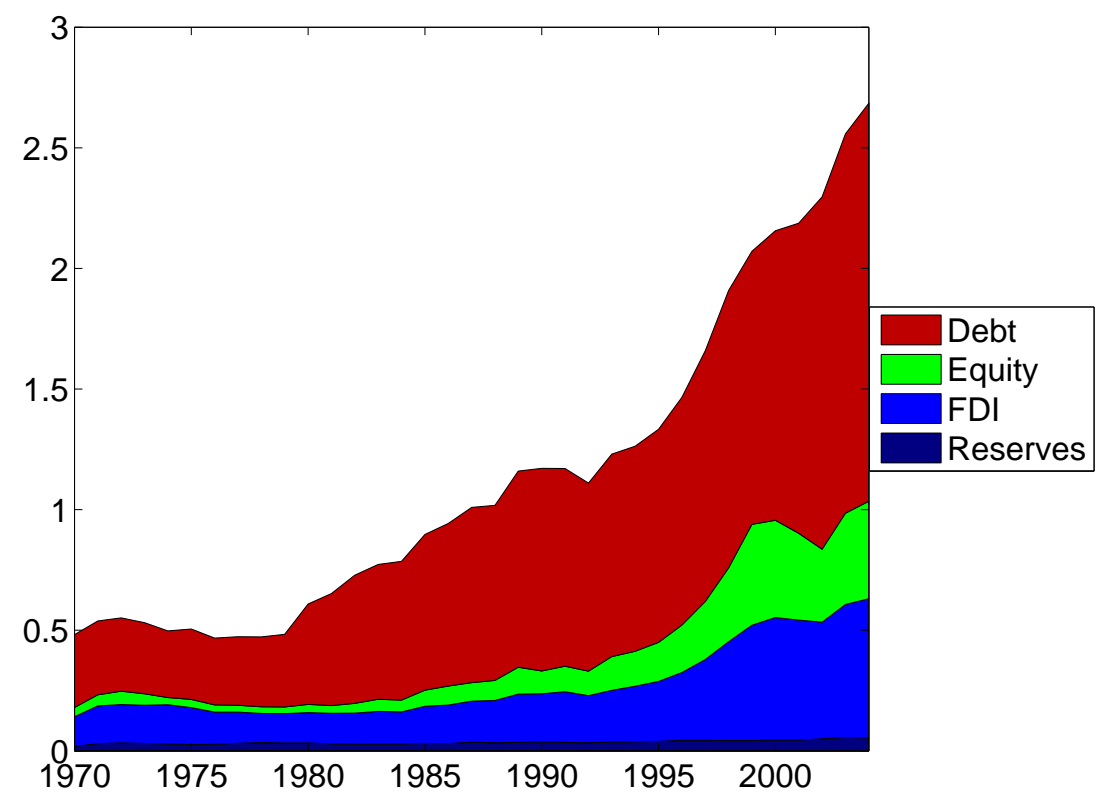

Notes: All positions are aggregates of absolute values of assets and liabilities divided by aggregate GDP across our sample of 71 countries.

Figure 2: Net Foreign Assets as a Ratio to GDP, 1970 to 2004

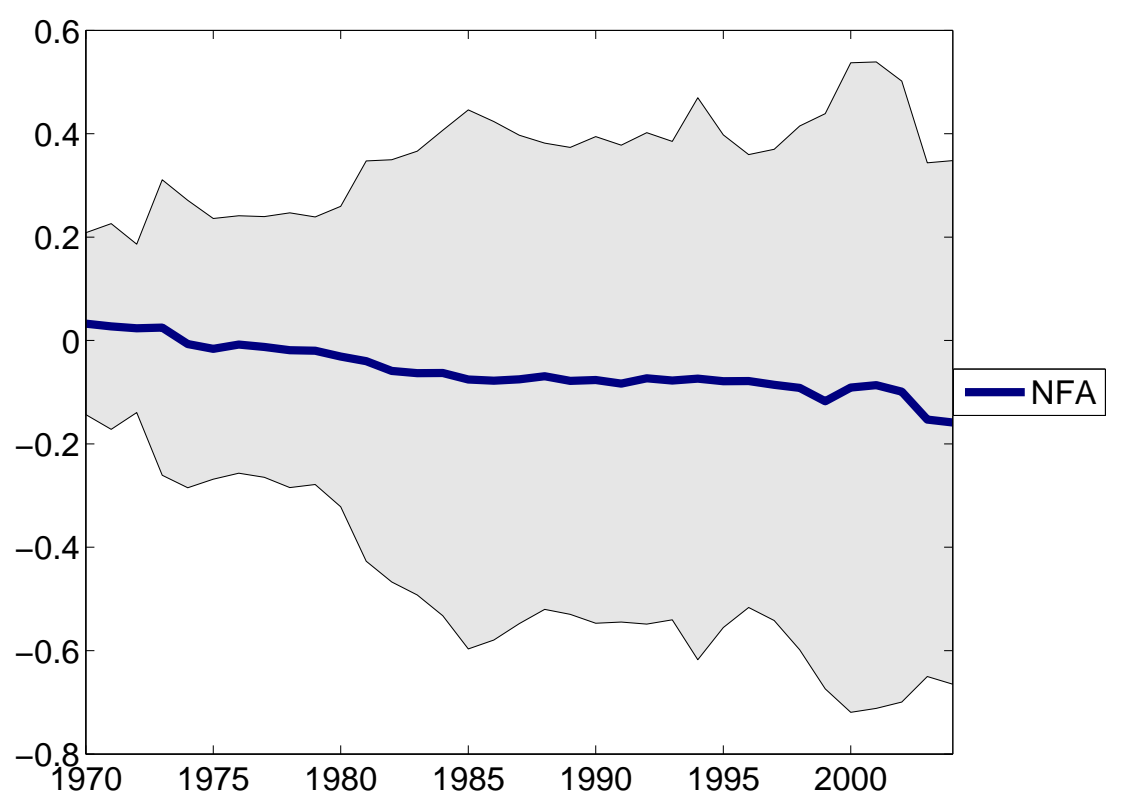

Notes: The solid line represents the aggregate of NFA divided by aggregate GDP across our sample of 71 countries, with the standard deviation across countries of the NFA to GDP ratio represented by the boundaries of the shaded area. 
Figure 3: Long-Run Coefficients for 71 Countries, 1970 to 2004: CPMG Approach

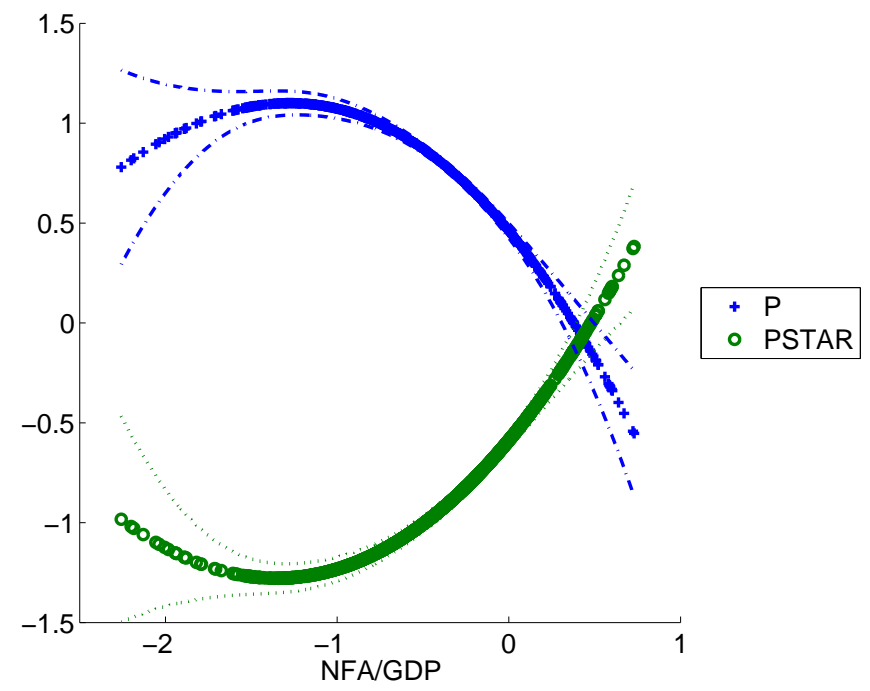

Notes: Estimates of the conditional long-run coefficients between the effective nominal exchange rate and domestic as well as weighted foreign prices in the panel ARDL model (27) using Chebyshev polynomials of order three in the conditioning variable, the latter defined as a 10-year predetermined moving average of the NFA to GDP ratio. The lag length is selected according to the Akaike Information Criterion with a maximum lag of 2. Standard error bands denote the $95 \%$ confidence intervals of the coefficient estimates.

Figure 4: Adjustment Coefficients for 71 Countries, 1970 to 2004: CPMG Approach

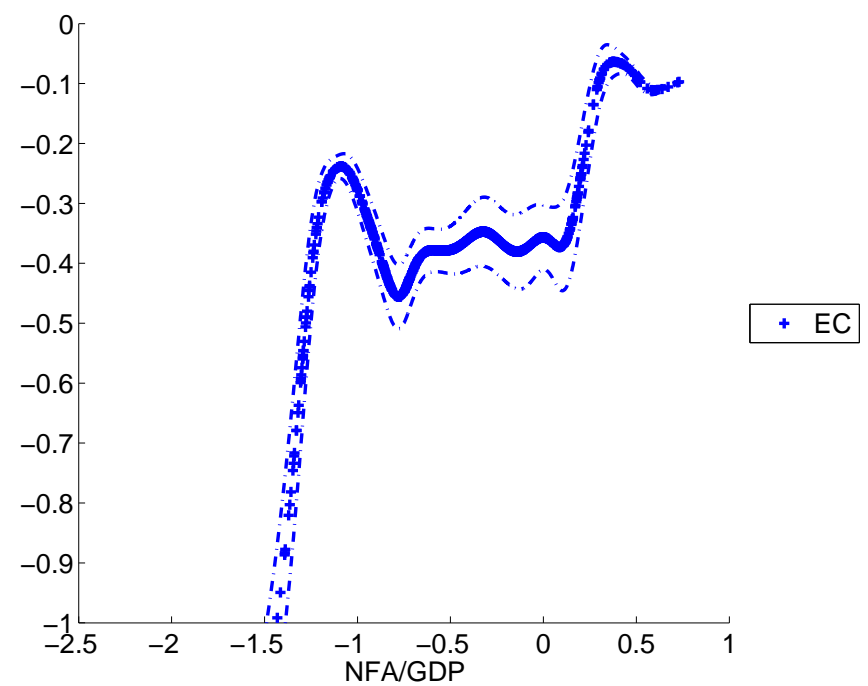

Notes: Smoothed mean group estimates of the adjustment coefficients in the panel ARDL model (27) using Chebyshev polynomials of order one in the conditioning variable, the latter defined as a 10-year predetermined moving average of the NFA to GDP ratio. The lag length is selected according to the Akaike Information Criterion with a maximum lag of 2 . Standard error bands denote the $95 \%$ confidence intervals of the coefficient estimates. 
Figure 5: Long-Run Coefficients for 71 Countries, 1970 to 2004: SKMG Approach

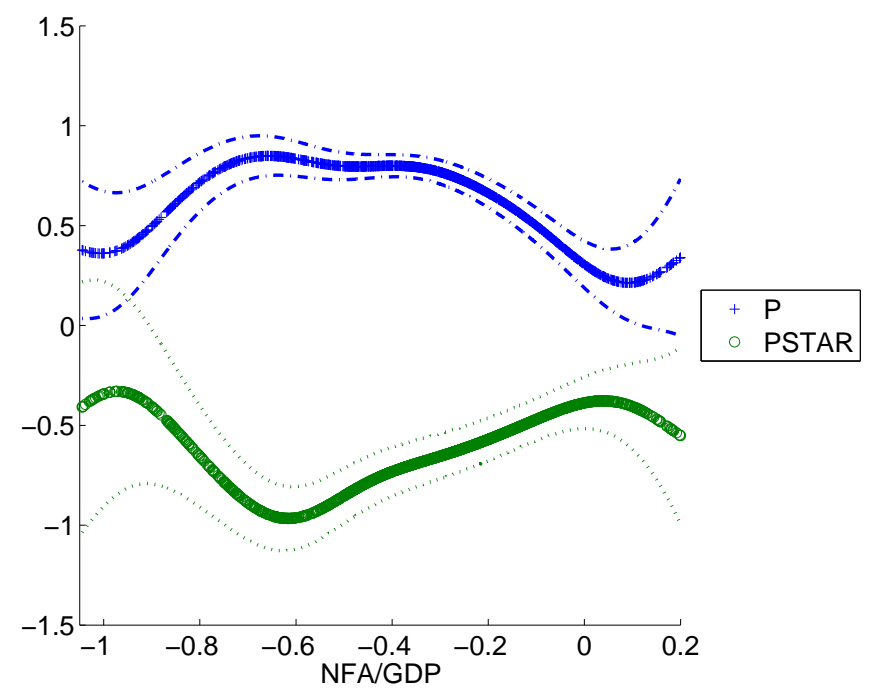

Notes: Estimates of the conditional long-run coefficients between the effective nominal exchange rate and domestic as well as weighted foreign prices in the panel ARDL model (27) using local kernels in the conditioning variable, the latter defined as a 10-year predetermined moving average of the NFA to GDP ratio. The lag length is selected according to the Akaike Information Criterion with a maximum lag of 2 . Standard error bands denote the $95 \%$ confidence intervals of the coefficient estimates.

Figure 6: Adjustment Coefficients for 71 Countries, 1970 to 2004: SKMG Approach

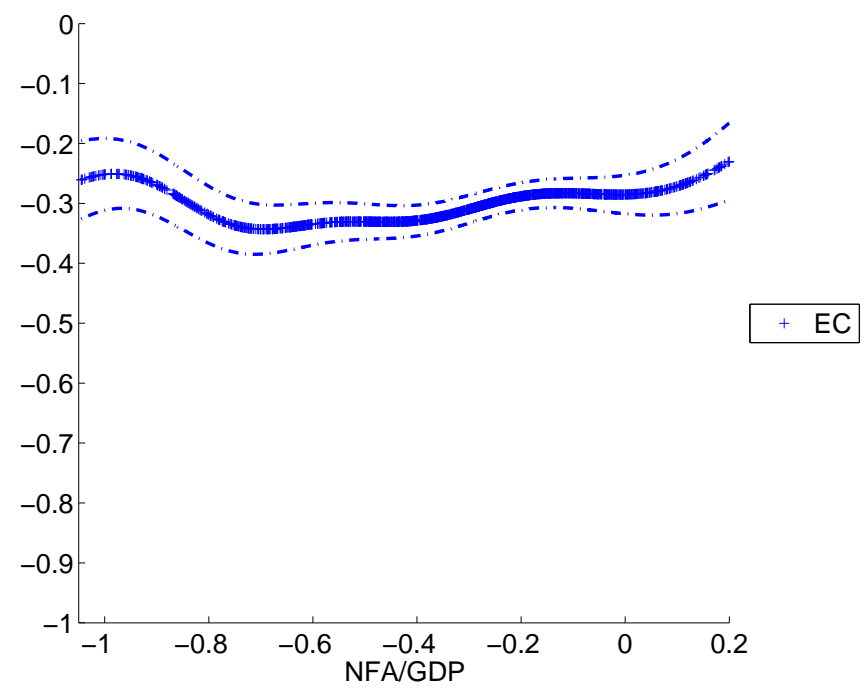

Notes: Estimates of the adjustment coefficients in the panel ARDL model (27) using local kernels in the conditioning variable, the latter defined as a 10-year predetermined moving average of the NFA to GDP ratio. The lag length is selected according to the Akaike Information Criterion with a maximum lag of 2. Standard error bands denote the $95 \%$ confidence intervals of the coefficient estimates. 
Figure 7: Long-Run Coefficients for 32 Industrial \& Emerging Market Economies, 1970 to 2004: CPMG Approach

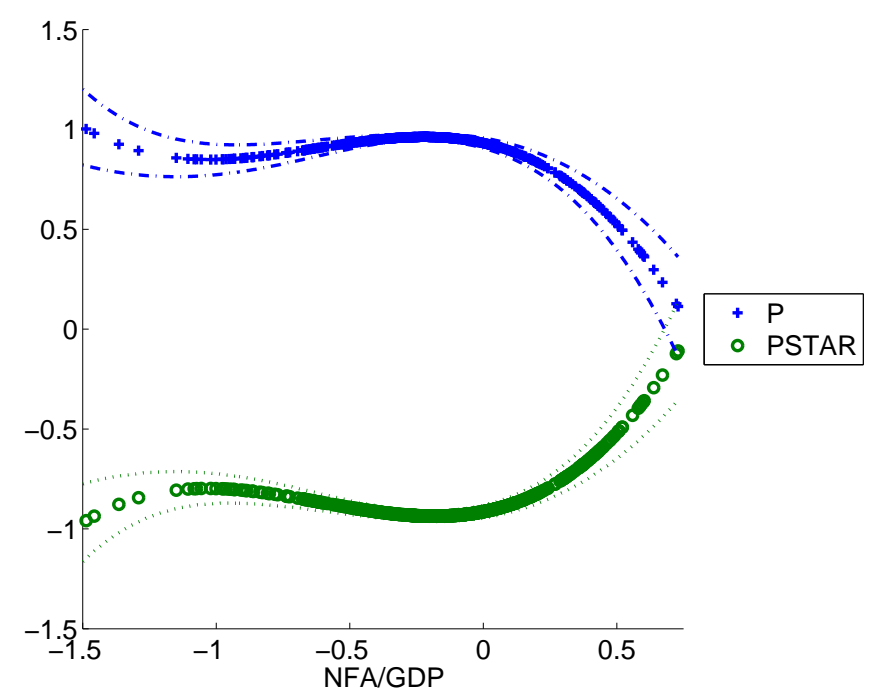

Notes: See Figure 3.

Figure 8: Long-Run Coefficients for 32 Industrial \& Emerging Market Economies, 1970 to 2004: SKMG Approach

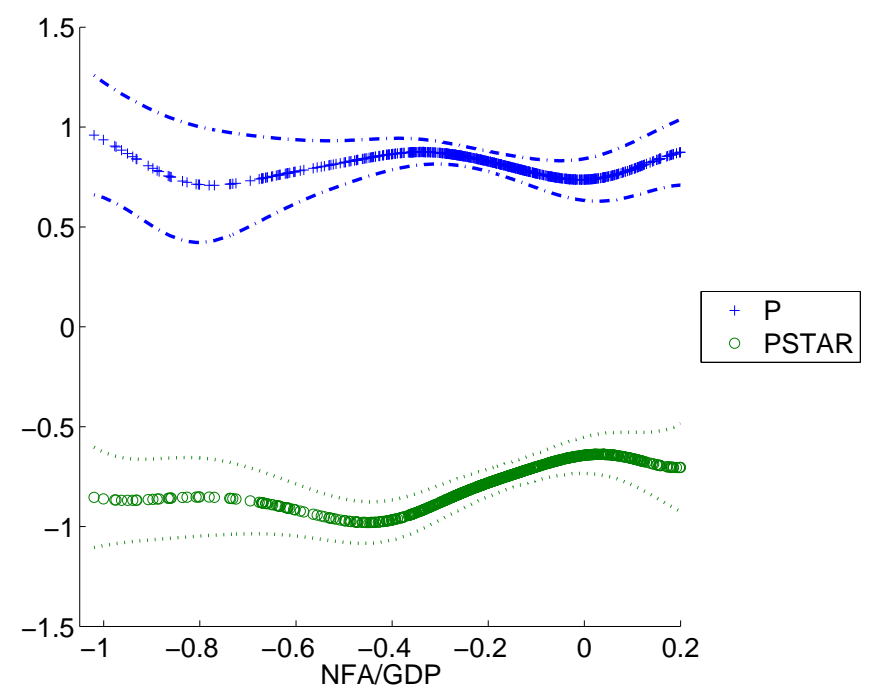

Notes: See Figure 5. 
Figure 9: Long-Run Coefficients for 39 Developing Countries, 1970 to 2004: CPMG Approach

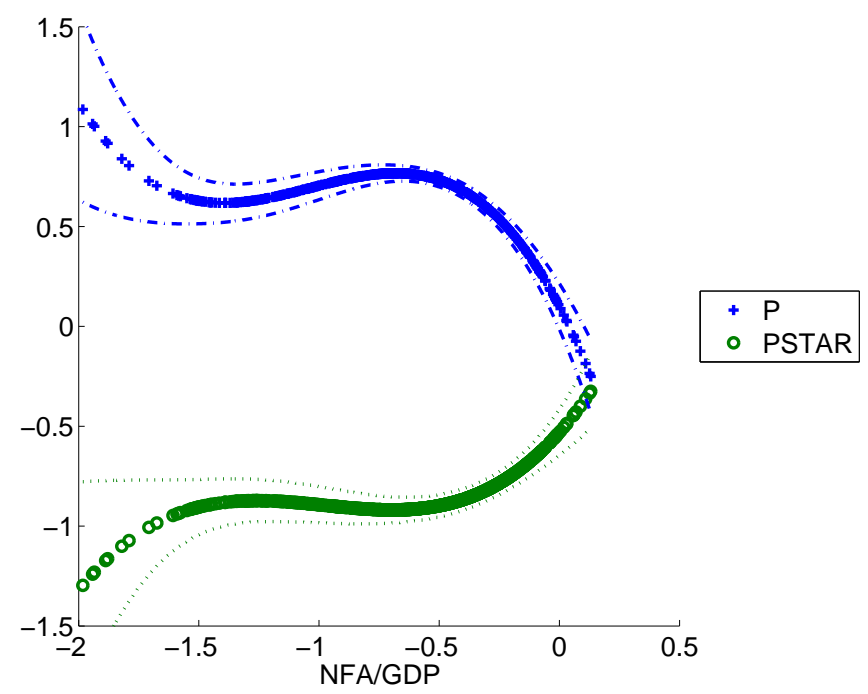

Notes: See Figure 3.

Figure 10: Long-Run Coefficients for 39 Developing Countries, 1970 to 2004: SKMG Approach

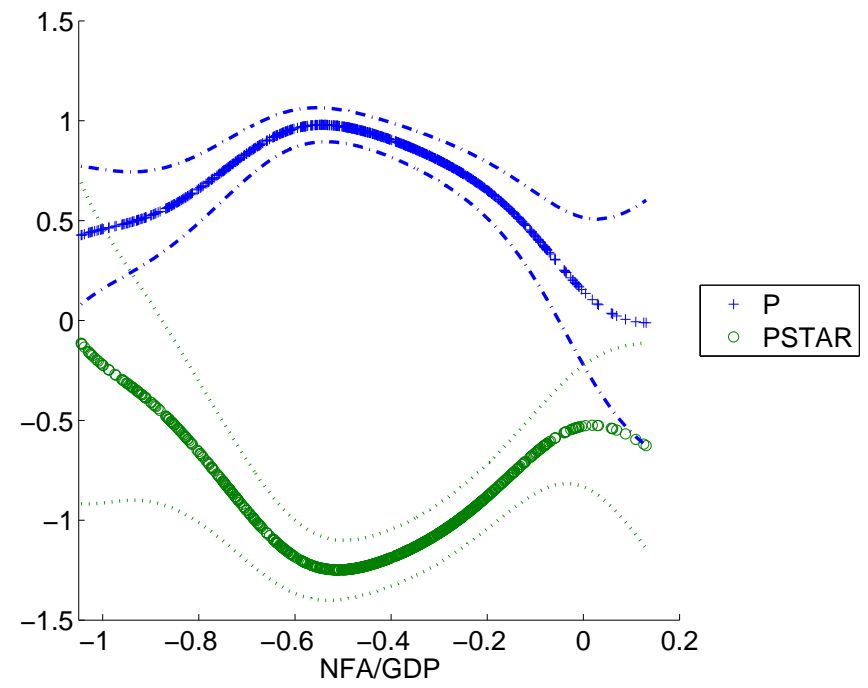

Notes: See Figure 5. 
Figure 11: Long-Run Coefficients for 50 Sticky Exchange Rate Currencies, 1970 to 2004: CPMG Approach

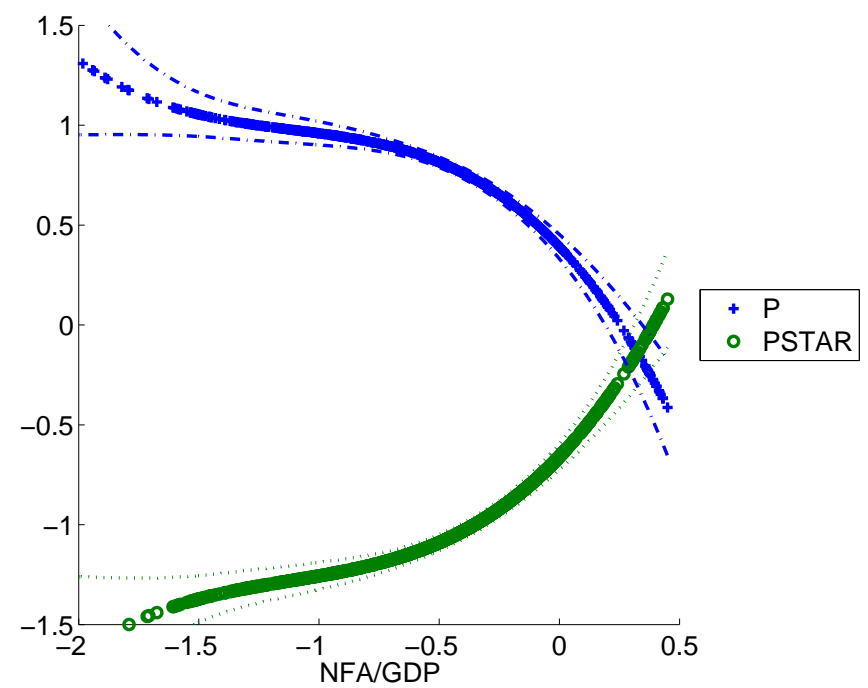

Notes: See Figure 3. A country is categorized as following a sticky exchange rate regime if its exchange rate classification code according to the recoded Levy-Yeyati and Sturzenegger (2005) data is on average larger than three over the sample period.

Figure 12: Long-Run Coefficients for 50 Sticky Exchange Rate Currencies, 1970 to 2004: SKMG Approach

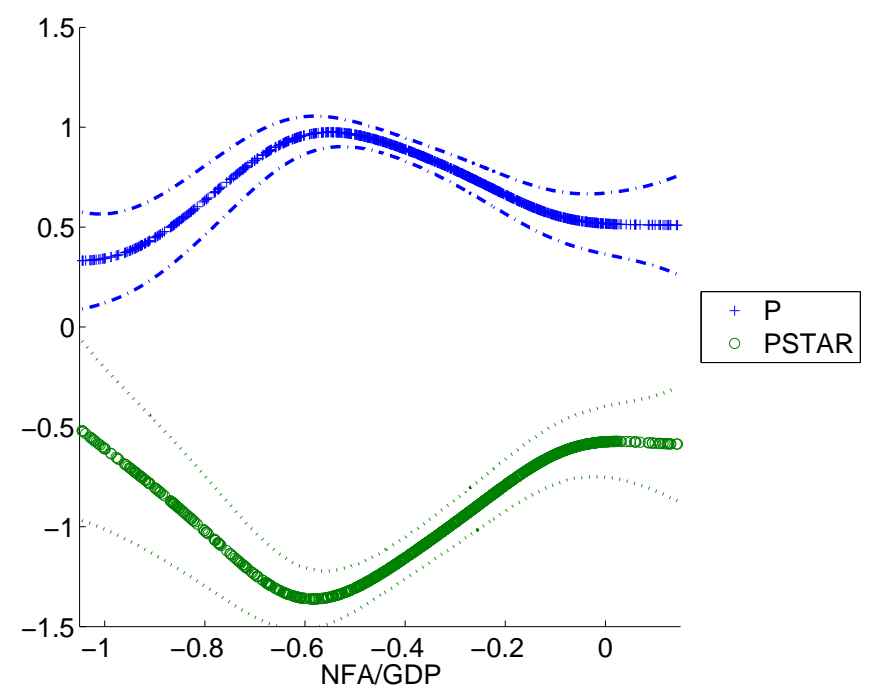

Notes: See Figures 5 and 11. 
Figure 13: Long-Run Coefficients for 21 Floating Exchange Rate Countries, 1970 to 2004:

CPMG Approach

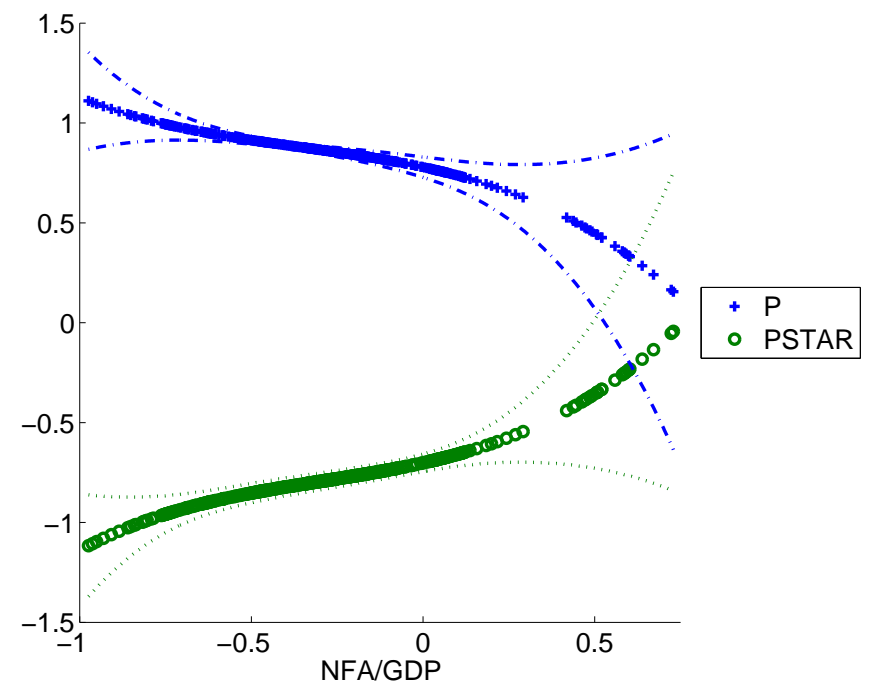

Notes: See Figure 3. A country is categorized as following a floating exchange rate regime if its exchange rate classification code according to the recoded Levy-Yeyati and Sturzenegger (2005) data is on average not larger than three over the sample period.

Figure 14: Long-Run Coefficients for 21 Floating Exchange Rate Countries, 1970 to 2004:

SKMG Approach

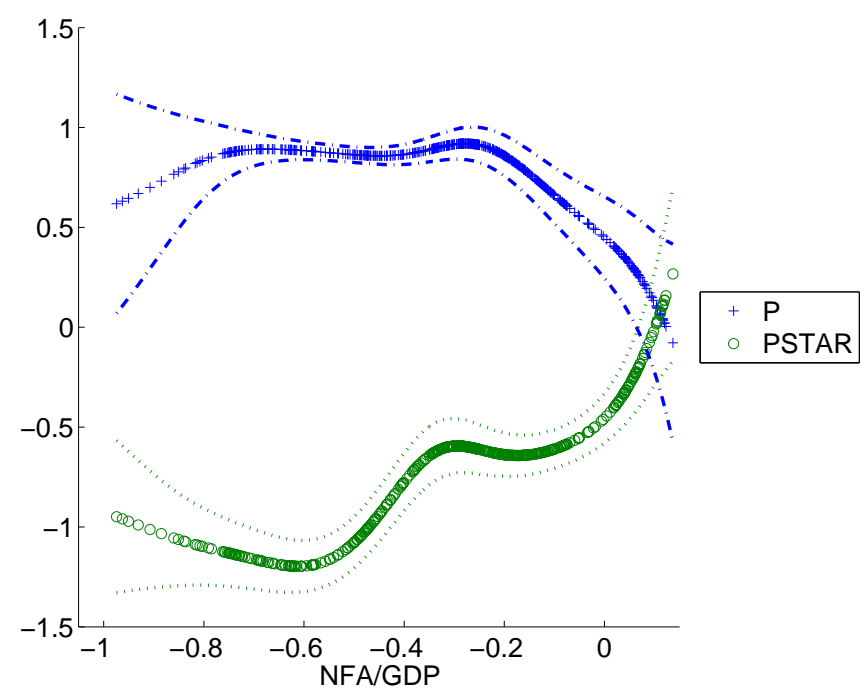

Notes: See Figures 5 and 13. 
Figure 15: Long-Run Coefficients for 39 Countries with High Degree of Price Variability, 1970 to 2004: CPMG Approach

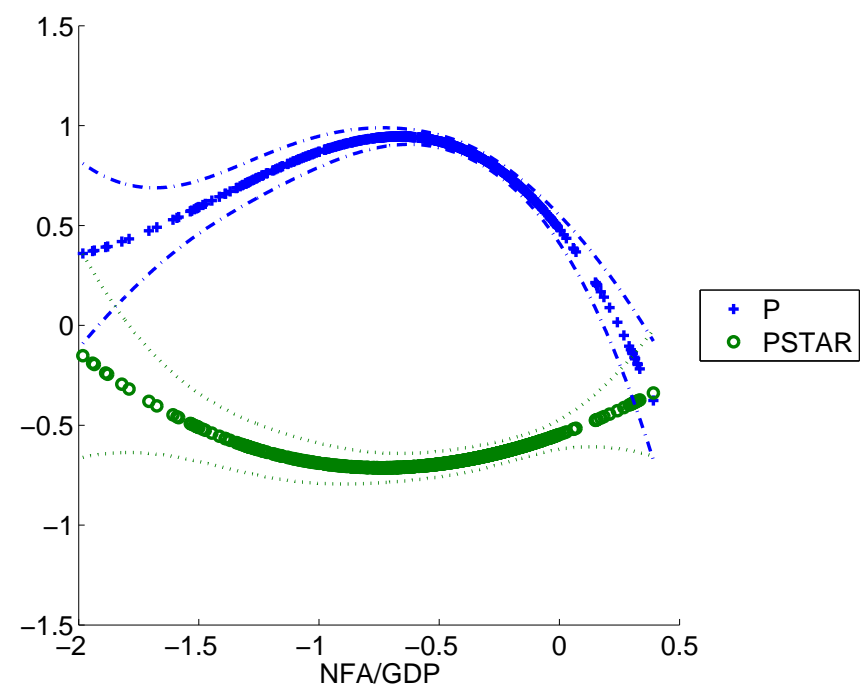

Notes: See Figure 3. A country is categorized as exhibiting a high degree of price variability if the annual consumer price index based rate of inflation on average exceeds eight percent over the sample period.

Figure 16: Long-Run Coefficients for 39 Countries with High Degree of Price Variability, 1970 to 2004: SKMG Approach

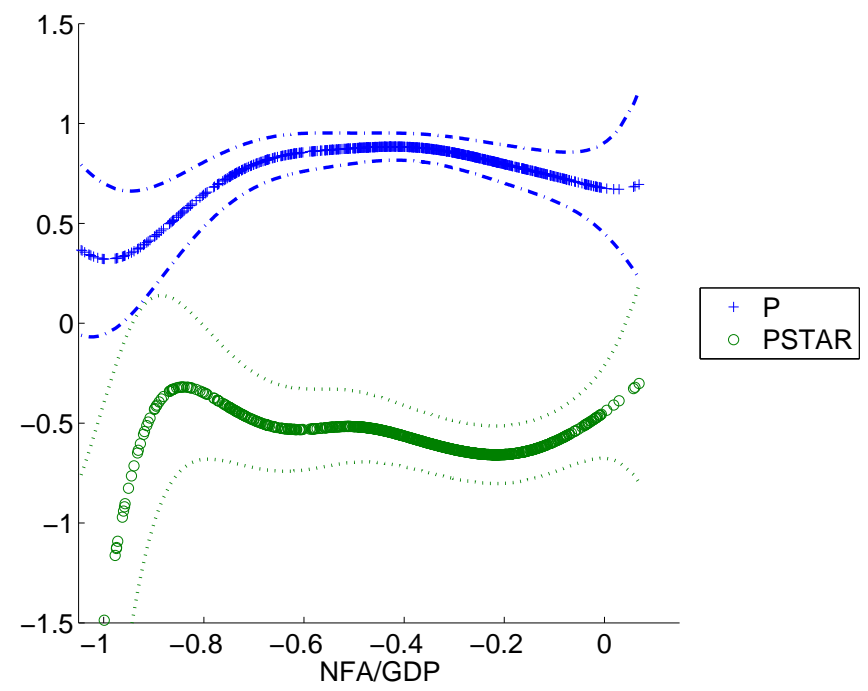

Notes: See Figures 5 and 15 . 
Figure 17: Long-Run Coefficients for 32 Countries with Low Degree of Price Variability, 1970 to 2004: CPMG Approach

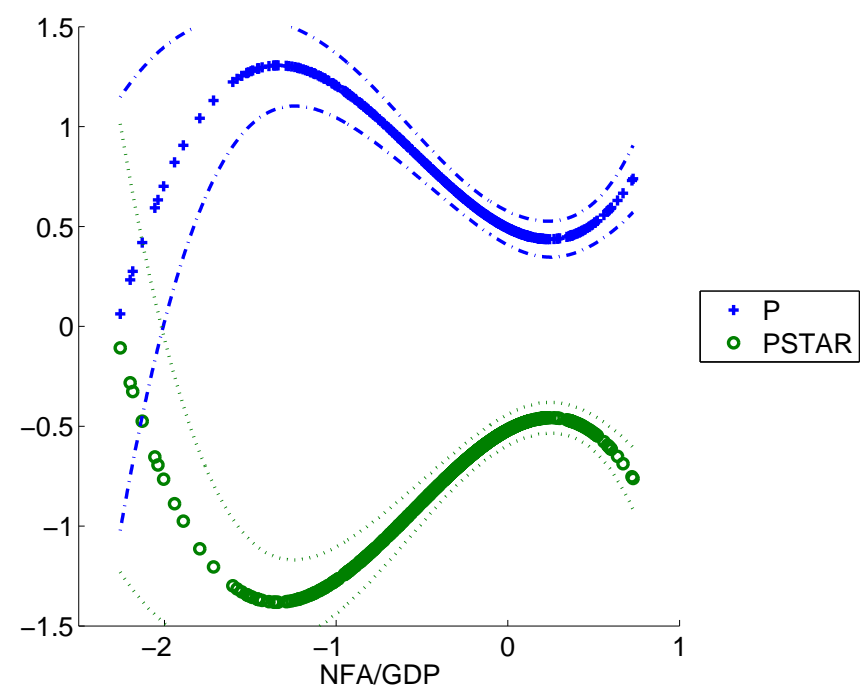

Notes: See Figure 3. A country is categorized as exhibiting a low degree of price variability if the annual consumer price index based rate of inflation on average does not exceed eight percent over the sample period.

Figure 18: Long-Run Coefficients for 32 Countries with Low Degree of Price Variability, 1970 to 2004: SKMG Approach

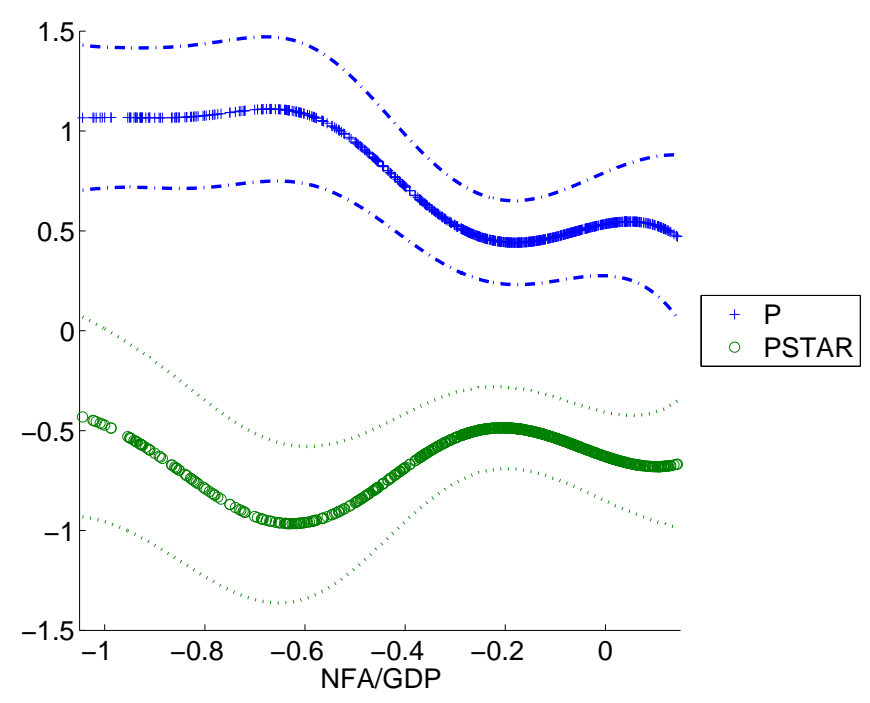

Notes: See Figures 5 and 17. 
Figure 19: Projections for the United States

\begin{tabular}{rrrr} 
& \multicolumn{1}{c}{$z^{(0)}$} & \multicolumn{1}{c}{$z^{(1)}$} & \multicolumn{1}{c}{$z^{(2)}$} \\
\hline$z$ & -0.28 & -0.48 & -0.69 \\
$\theta_{1}$ & 0.72 & 0.86 & 0.97 \\
$\theta_{2}$ & -0.84 & -1.00 & -1.11 \\
$\alpha$ & -0.50 & -0.67 & -0.85 \\
\hline \hline
\end{tabular}

(a) Counterfactual States

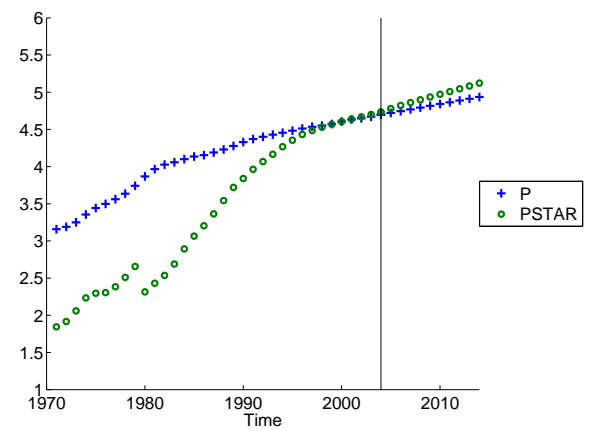

(b) Explanatory Variables

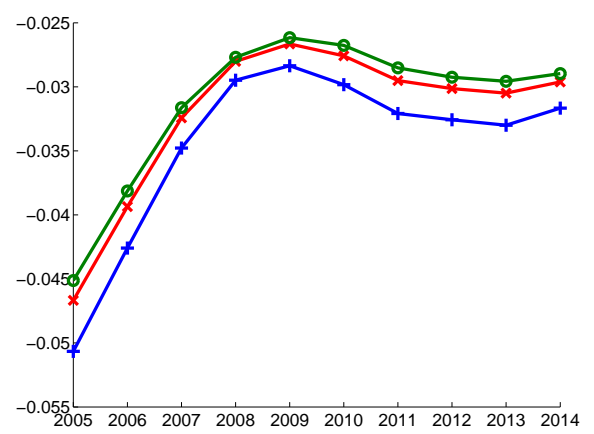

(d) Nominal Exchange Rate Changes

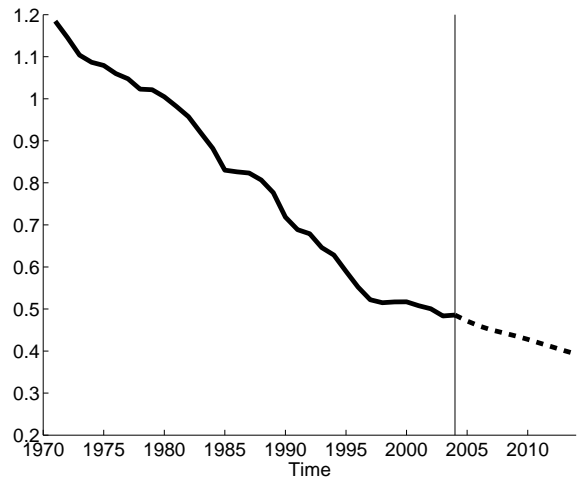

(c) Common Factor Effect

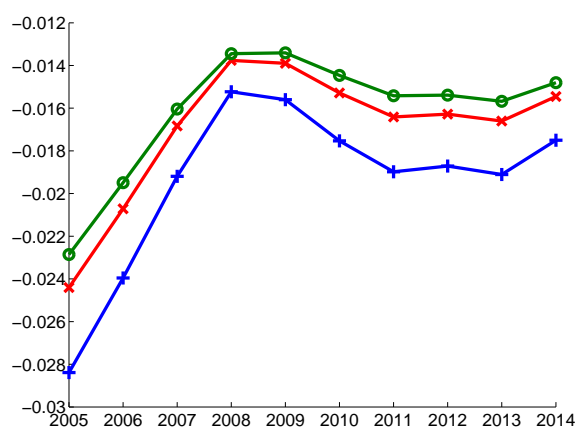

(e) Real Exchange Rate Changes

Notes: Sub-figure (a): Alternative scenarios for the state of the international investment position and their implications for the long-run coefficients and the speed of adjustment coefficient. Subfigures (b) and (c): Values to the left of the vertical line denote actual observations for domestic and weighted foreign prices as well as the common factor effects $\left(\hat{\boldsymbol{\chi}}_{i}^{\prime} \boldsymbol{g}_{t}\right)$; values to the right of the vertical line represent trend extrapolations for these variables based on 10-year moving averages of past values. Sub-figures $(d)$ and $(e)$ : The $(+)$-symbol blue line represents projections based on trend extrapolations of domestic and weighted foreign prices as well as the common factors conditional on the 2004 level of the NFA to GDP ratio $\left(z^{(0)}\right)$; the $(\times)$-symbol red line represents projections based on trend extrapolations of the same variables conditional on an NFA to GDP ratio equal to $z^{(1)}$; the (o)-symbol green line represents projections based on trend extrapolations of the same variables conditional on an NFA to GDP ratio equal to $z^{(2)}$. 
Figure 20: Projections for the United Kingdom

\begin{tabular}{rrrr} 
& \multicolumn{1}{c}{$z^{(0)}$} & \multicolumn{1}{c}{$z^{(1)}$} & \multicolumn{1}{c}{$z^{(2)}$} \\
\hline$z$ & -0.15 & -0.30 & -0.45 \\
$\theta_{1}$ & 0.61 & 0.73 & 0.84 \\
$\theta_{2}$ & -0.74 & -0.86 & -0.97 \\
$\alpha$ & -0.26 & -0.26 & -0.27 \\
\hline \hline
\end{tabular}

(a) Counterfactual States

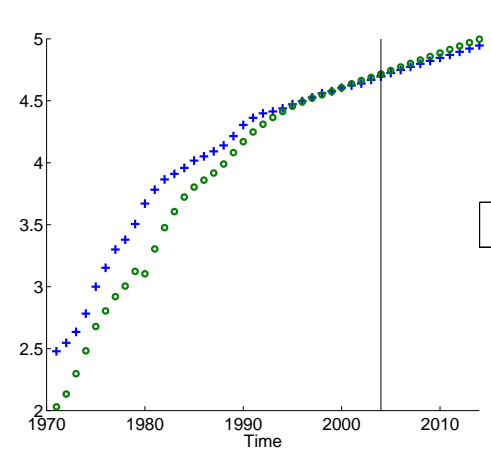

(b) Explanatory Variables

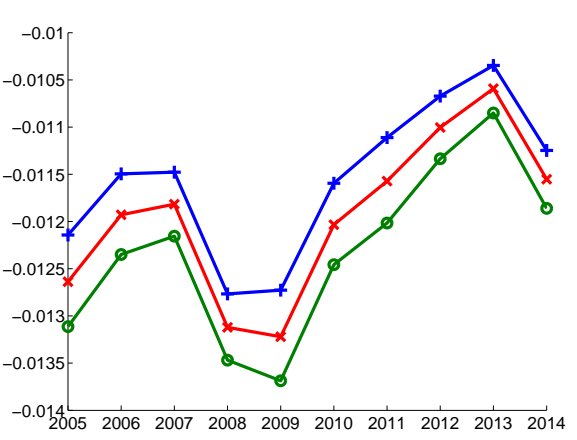

(d) Nominal Exchange Rate Changes

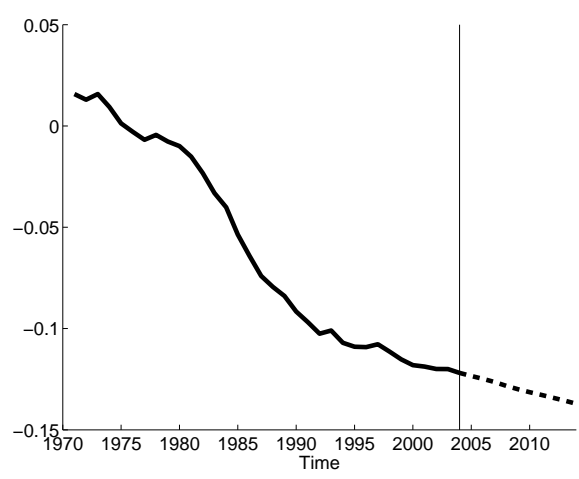

(c) Common Factor Effect

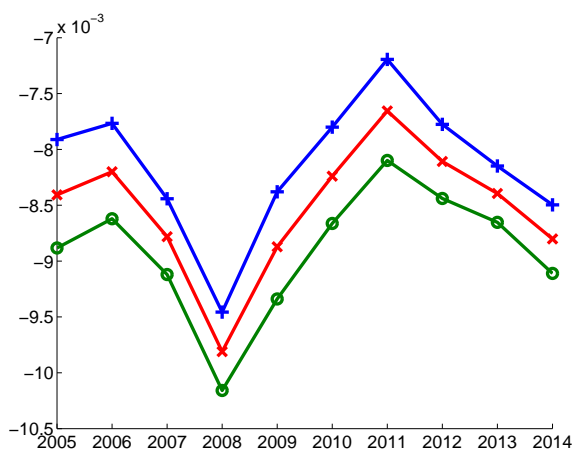

(e) Real Exchange Rate Changes

Notes: See Figure 19. 
Figure 21: Projections for Spain

\begin{tabular}{rrrr} 
& \multicolumn{1}{c}{$z^{(0)}$} & \multicolumn{1}{c}{$z^{(1)}$} & \multicolumn{1}{c}{$z^{(2)}$} \\
\hline$z$ & -0.18 & -0.25 & -0.33 \\
$\theta_{1}$ & 0.63 & 0.70 & 0.76 \\
$\theta_{2}$ & -0.76 & -0.82 & -0.88 \\
$\alpha$ & -0.27 & -0.25 & -0.23 \\
\hline \hline
\end{tabular}

(a) Counterfactual States

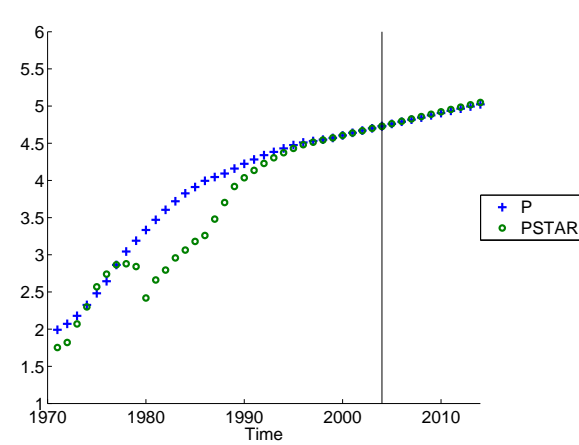

(b) Explanatory Variables

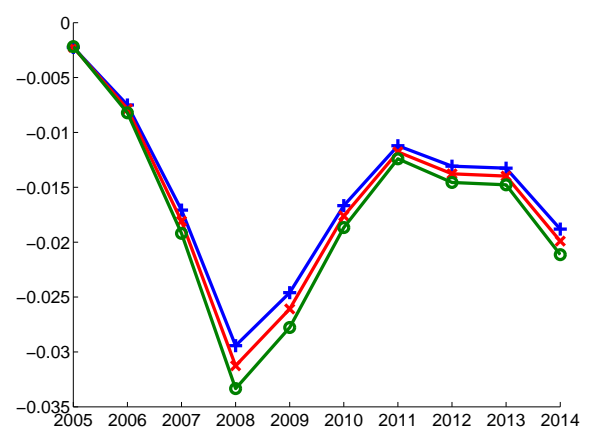

(d) Nominal Exchange Rate Changes

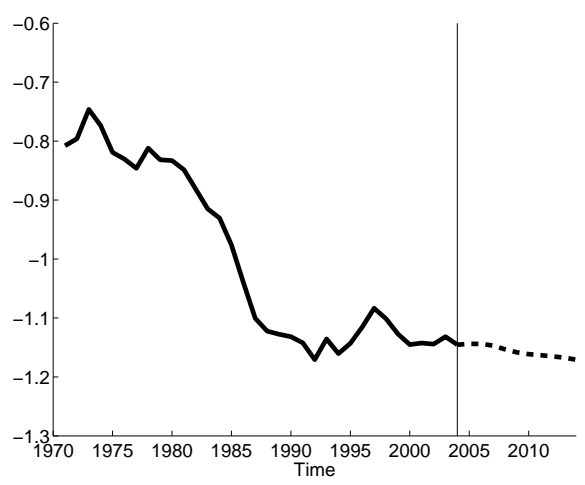

(c) Common Factor Effect

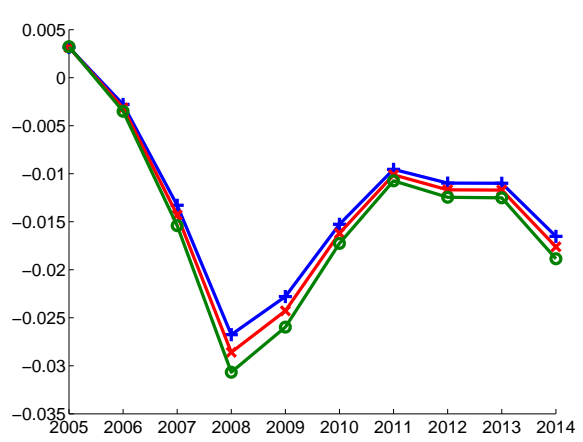

(e) Real Exchange Rate Changes

Notes: See Figure 19 
Figure 22: Projections for Mexico

\begin{tabular}{rrrr} 
& \multicolumn{1}{c}{$z^{(0)}$} & \multicolumn{1}{c}{$z^{(1)}$} & \multicolumn{1}{c}{$z^{(2)}$} \\
\hline$z$ & -0.44 & -0.54 & -0.64 \\
$\theta_{1}$ & 0.83 & 0.89 & 0.95 \\
$\theta_{2}$ & -0.96 & -1.03 & -1.09 \\
$\alpha$ & -0.60 & -0.68 & -0.76 \\
\hline \hline
\end{tabular}

(a) Counterfactual States

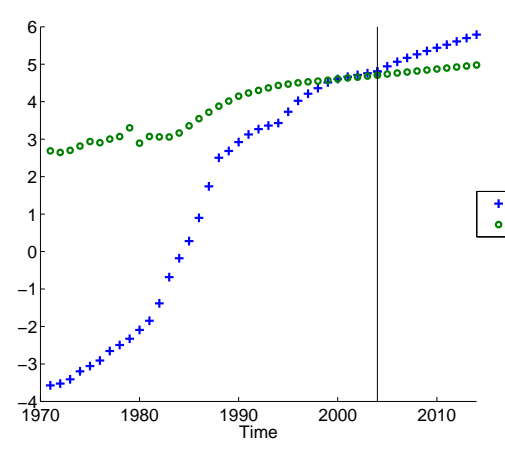

(b) Explanatory Variables

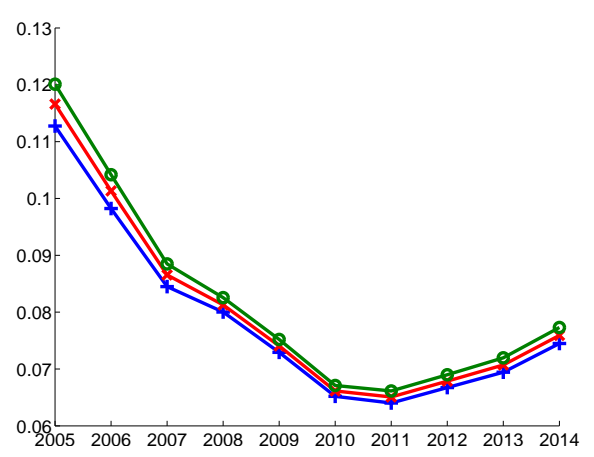

(d) Nominal Exchange Rate Changes

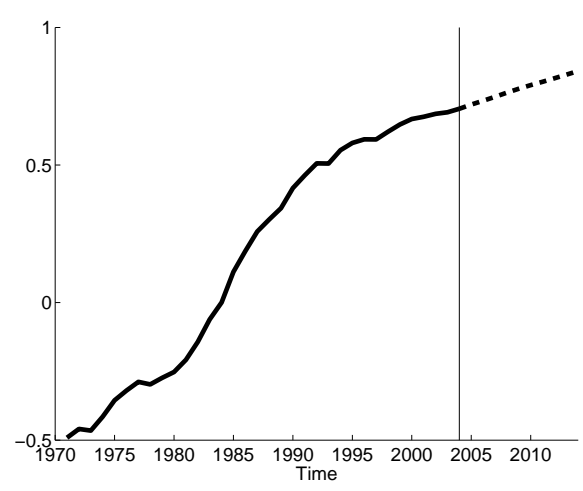

(c) Common Factor Effect

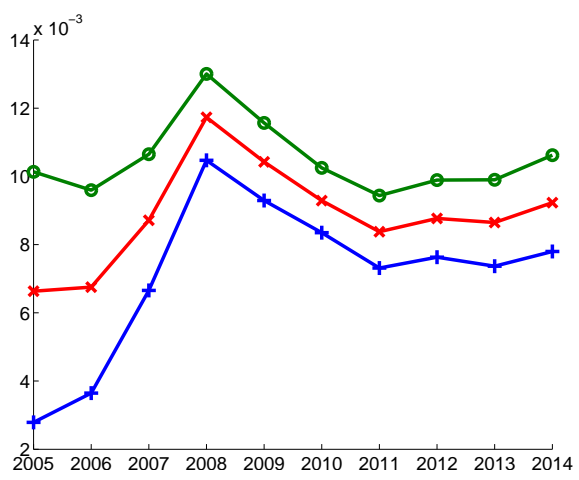

(e) Real Exchange Rate Changes

Notes: See Figure 19. 
Figure 23: Projections for Turkey

\begin{tabular}{rrrr} 
& \multicolumn{1}{c}{$z^{(0)}$} & \multicolumn{1}{c}{$z^{(1)}$} & \multicolumn{1}{c}{$z^{(2)}$} \\
\hline$z$ & -0.22 & -0.29 & -0.35 \\
$\theta_{1}$ & 0.67 & 0.72 & 0.77 \\
$\theta_{2}$ & -0.80 & -0.85 & -0.90 \\
$\alpha$ & -0.38 & -0.40 & -0.43 \\
\hline \hline
\end{tabular}

(a) Counterfactual States

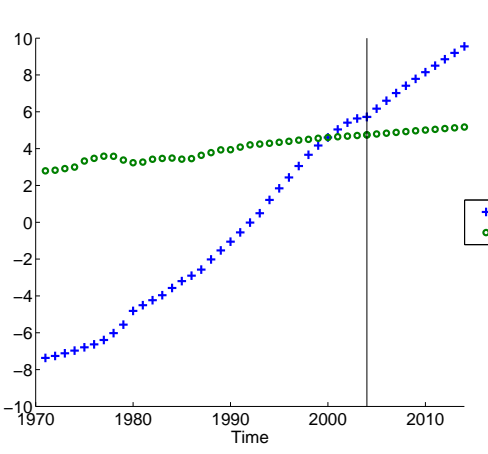

(b) Explanatory Variables

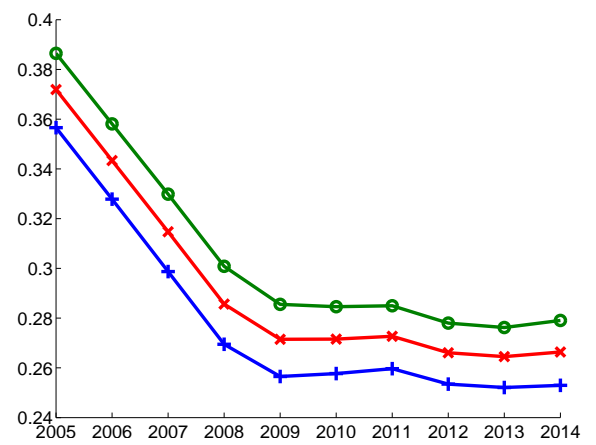

(d) Nominal Exchange Rate Changes

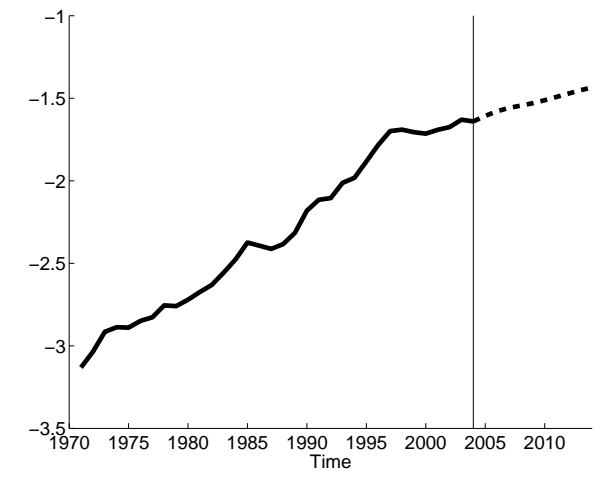

(c) Common Factor Effect

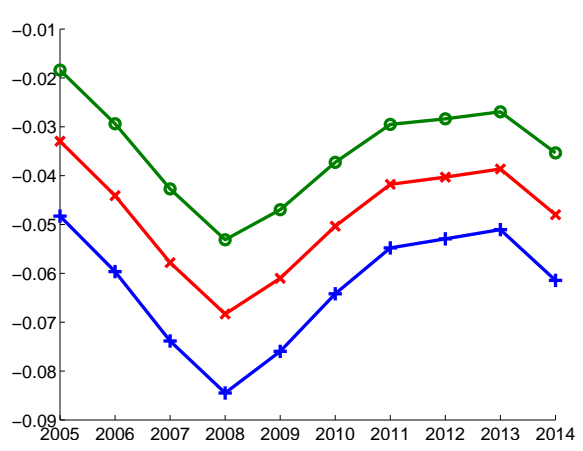

(e) Real Exchange Rate Changes

Notes: See Figure 19. 
Figure 24: Projections for Uruguay

\begin{tabular}{rrrr} 
& \multicolumn{1}{c}{$z^{(0)}$} & \multicolumn{1}{c}{$z^{(1)}$} & \multicolumn{1}{c}{$z^{(2)}$} \\
\hline$z$ & -0.23 & -0.42 & -0.60 \\
$\theta_{1}$ & 0.68 & 0.82 & 0.93 \\
$\theta_{2}$ & -0.80 & -0.95 & -1.07 \\
$\alpha$ & -0.49 & -0.69 & -0.89 \\
\hline \hline
\end{tabular}

(a) Counterfactual States

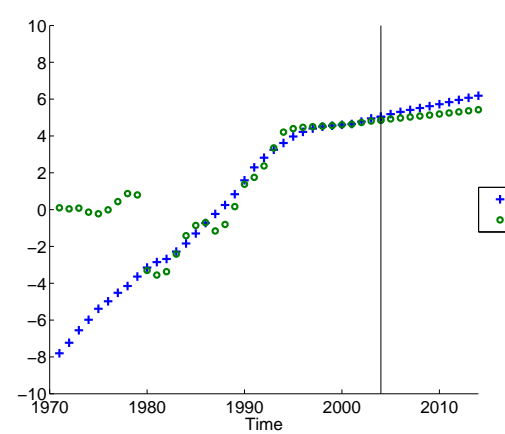

(b) Explanatory Variables

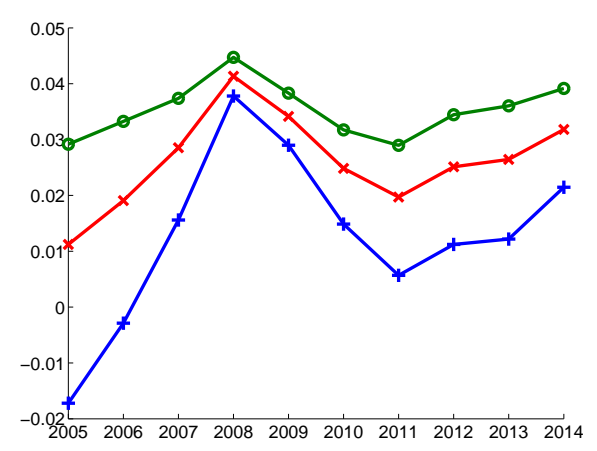

(d) Nominal Exchange Rate Changes

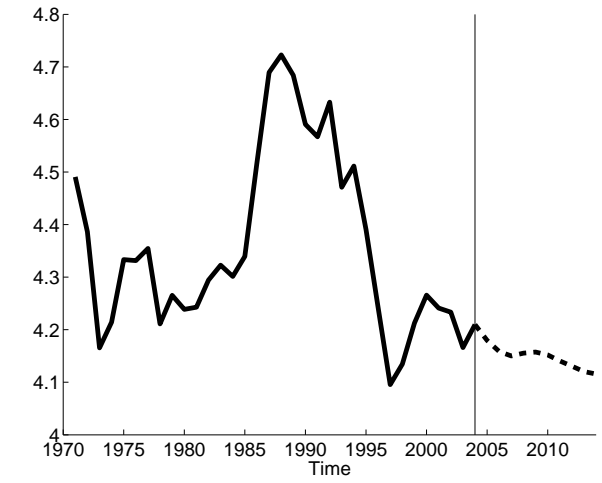

(c) Common Factor Effect

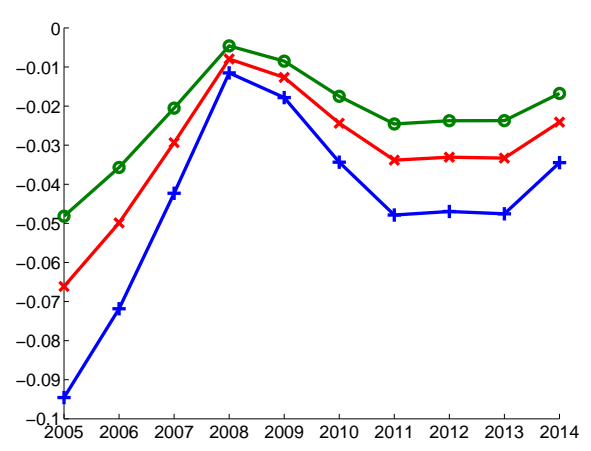

(e) Real Exchange Rate Changes

Notes: See Figure 19. 
Figure 25: Projections for India

\begin{tabular}{rrrr} 
& \multicolumn{1}{c}{$z^{(0)}$} & \multicolumn{1}{c}{$z^{(1)}$} & \multicolumn{1}{c}{$z^{(2)}$} \\
\hline$z$ & -0.19 & -0.15 & -0.11 \\
$\theta_{1}$ & 0.65 & 0.61 & 0.57 \\
$\theta_{2}$ & -0.77 & -0.74 & -0.70 \\
$\alpha$ & -0.20 & -0.21 & -0.23 \\
\hline \hline
\end{tabular}

(a) Counterfactual States

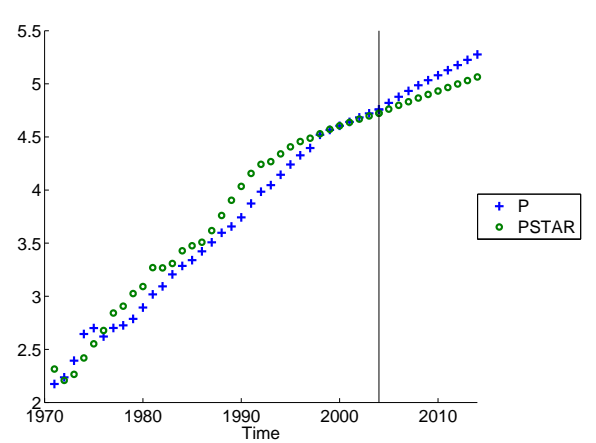

(b) Explanatory Variables

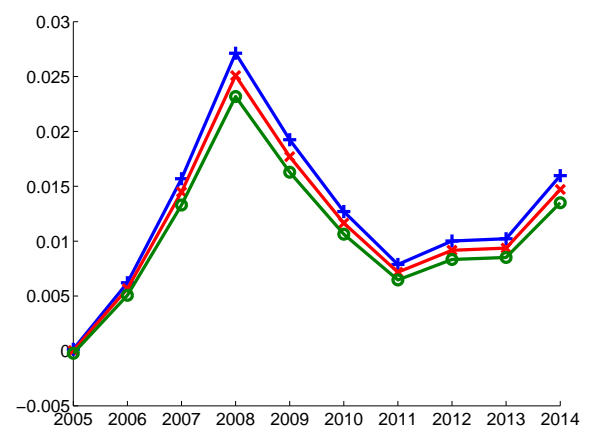

(d) Nominal Exchange Rate Changes

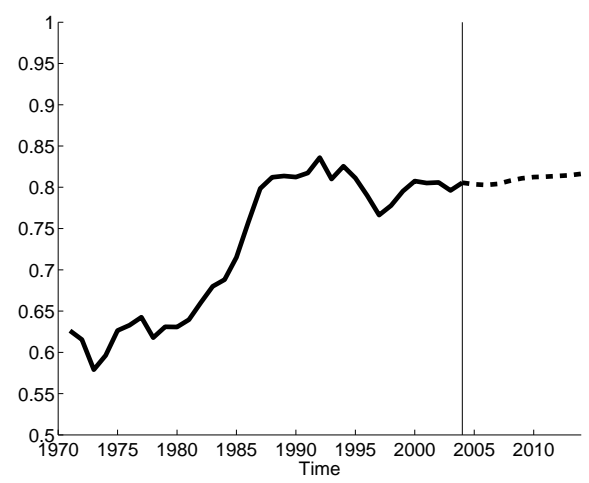

(c) Common Factor Effect

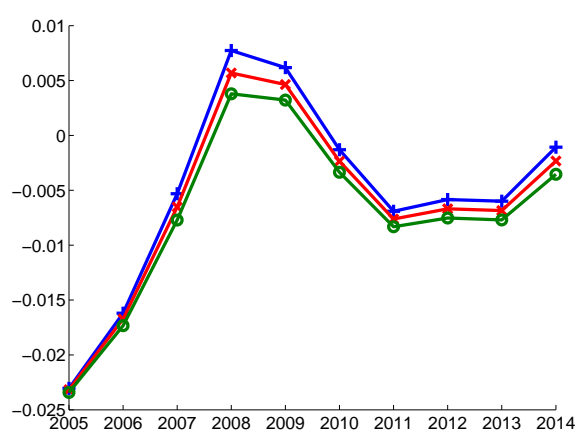

(e) Real Exchange Rate Changes

Notes: See Figure 19. 
Figure 26: Projections for Paraguay

\begin{tabular}{rrrr} 
& \multicolumn{1}{c}{$z^{(0)}$} & \multicolumn{1}{c}{$z^{(1)}$} & \multicolumn{1}{c}{$z^{(2)}$} \\
\hline$z$ & -0.55 & -0.83 & -1.12 \\
$\theta_{1}$ & 0.90 & 1.03 & 1.09 \\
$\theta_{2}$ & -1.04 & -1.18 & -1.26 \\
$\alpha$ & -0.37 & -0.39 & -0.40 \\
\hline \hline
\end{tabular}

(a) Counterfactual States

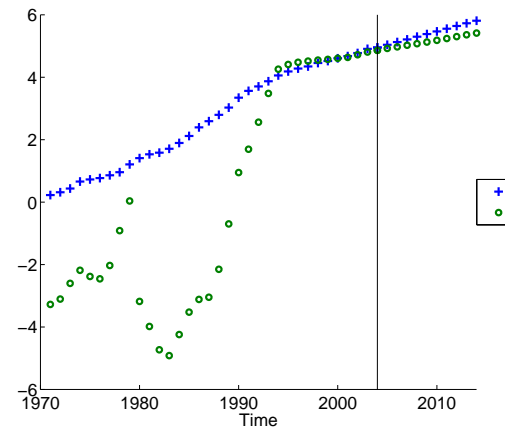

(b) Explanatory Variables

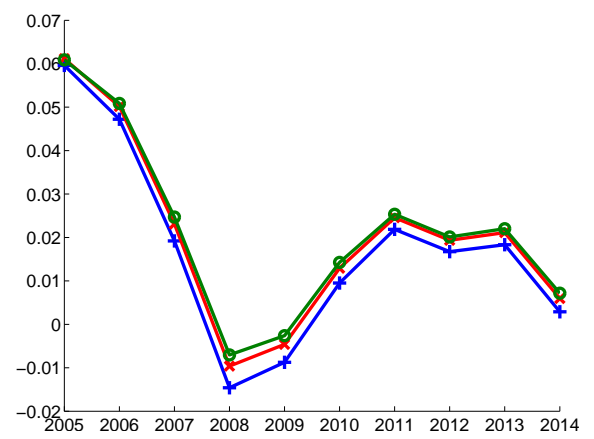

(d) Nominal Exchange Rate Changes

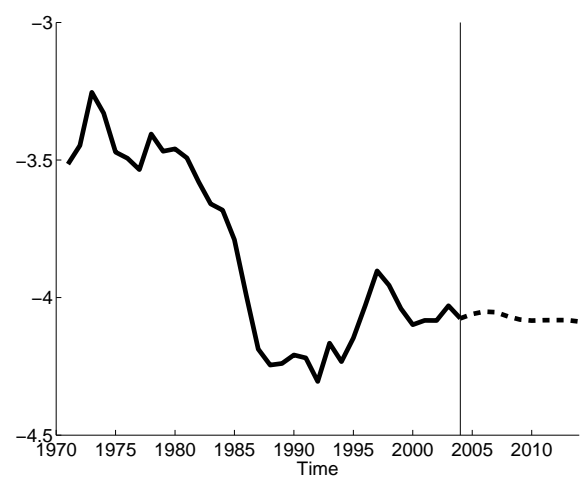

(c) Common Factor Effect

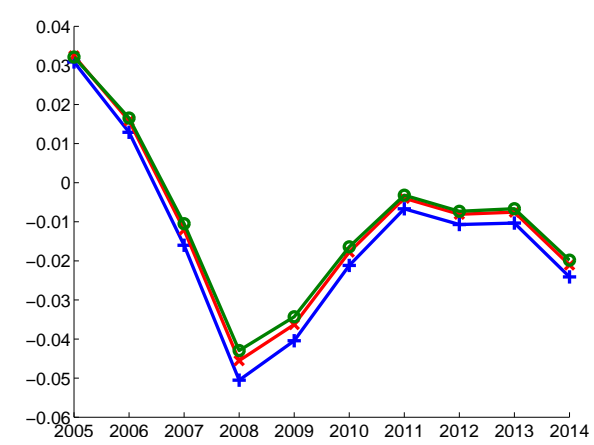

(e) Real Exchange Rate Changes

Notes: See Figure 19. 
Figure 27: Projections for Thailand

\begin{tabular}{rrrr} 
& \multicolumn{1}{c}{$z^{(0)}$} & \multicolumn{1}{c}{$z^{(1)}$} & \multicolumn{1}{c}{$z^{(2)}$} \\
\hline$z$ & -0.44 & -0.41 & -0.38 \\
$\theta_{1}$ & 0.84 & 0.81 & 0.79 \\
$\theta_{2}$ & -0.97 & -0.95 & -0.92 \\
$\alpha$ & -0.19 & -0.18 & -0.16 \\
\hline \hline
\end{tabular}

(a) Counterfactual States

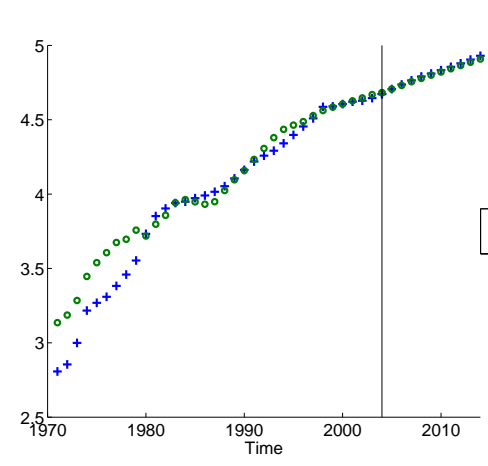

(b) Explanatory Variables

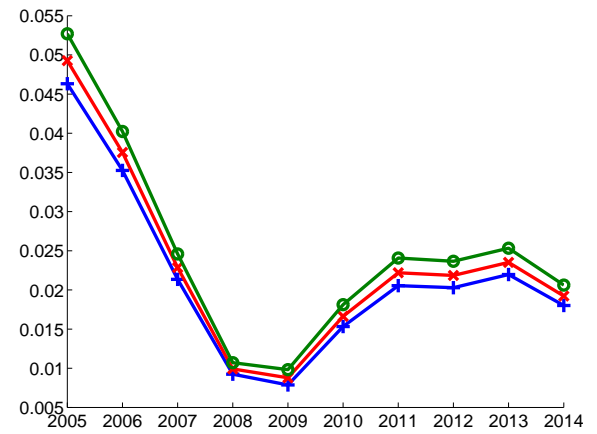

(d) Nominal Exchange Rate Changes

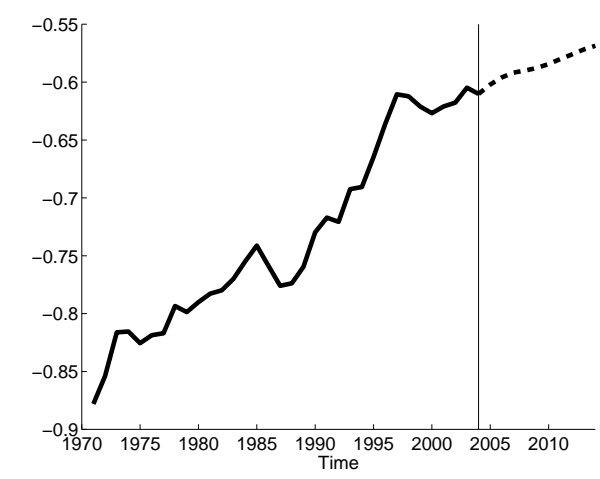

(c) Common Factor Effect

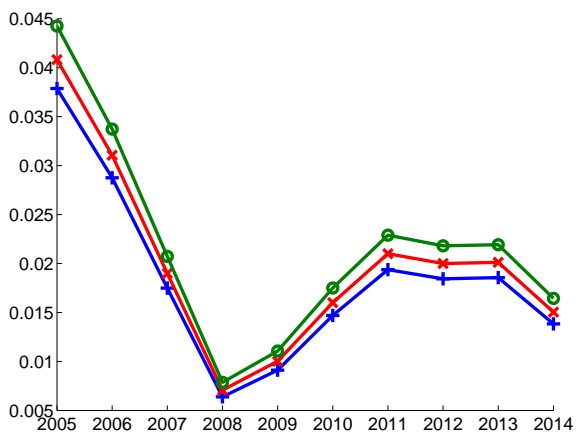

(e) Real Exchange Rate Changes

Notes: See Figure 19. 


\section{Appendices}

\section{A Two-Step Estimation of the CPMG Model}

In this appendix, we wish to derive the stacked representation in Equation (15). To this purpose, we rewrite Equation (14) as

$$
\begin{aligned}
\Delta \boldsymbol{y}_{i} & =\boldsymbol{A}_{i}\left(\tilde{\boldsymbol{z}}_{i}\right) \boldsymbol{y}_{i,-1}+\boldsymbol{B}_{i}\left(\tilde{\boldsymbol{z}}_{i}\right) \tilde{\boldsymbol{x}}_{i}+\boldsymbol{\Psi}_{i}\left(\tilde{\boldsymbol{z}}_{i}\right) \tilde{\boldsymbol{h}}_{i}+\boldsymbol{\varepsilon}_{i} \\
& =\boldsymbol{A}_{i}\left(\tilde{\boldsymbol{z}}_{i}\right)\left[\boldsymbol{y}_{i,-1}+\boldsymbol{A}_{i}\left(\tilde{\boldsymbol{z}}_{i}\right)^{-1} \boldsymbol{B}_{i}\left(\tilde{\boldsymbol{z}}_{i}\right) \tilde{\boldsymbol{x}}_{i}\right]+\boldsymbol{\Psi}_{i}\left(\tilde{\boldsymbol{z}}_{i}\right) \tilde{\boldsymbol{h}}_{i}+\boldsymbol{\varepsilon}_{i} \\
& =\boldsymbol{A}_{i}\left(\tilde{\boldsymbol{z}}_{i}\right)\left[\boldsymbol{y}_{i,-1}-\boldsymbol{\Theta}_{i}\left(\tilde{\boldsymbol{z}}_{i}\right) \tilde{\boldsymbol{x}}_{i}\right]+\boldsymbol{\Psi}_{i}\left(\tilde{\boldsymbol{z}}_{i}\right) \tilde{\boldsymbol{h}}_{i}+\boldsymbol{\varepsilon}_{i},
\end{aligned}
$$

where $\tilde{\boldsymbol{x}}_{i}=\left(\boldsymbol{x}_{1 i}^{\prime} \boldsymbol{x}_{2 i}^{\prime} \ldots \boldsymbol{x}_{m i}^{\prime}\right)^{\prime}, \boldsymbol{x}_{\ell i}=\left(x_{\ell i 1} x_{\ell i 2} \ldots x_{\ell i T_{i}}\right)^{\prime}, \ell=1,2, \ldots, m, \tilde{\boldsymbol{h}}_{i}=\left(\boldsymbol{h}_{1 i}^{\prime} \boldsymbol{h}_{2 i}^{\prime} \ldots \boldsymbol{h}_{n i}^{\prime}\right)^{\prime}$, $\boldsymbol{h}_{\ell i}=\left(h_{\ell i 1} h_{\ell i 2} \ldots h_{\ell i T_{i}}\right)^{\prime}, \ell=1,2, \ldots, n, n=2 p+1+m(2 q+1)$, and the coefficients are specified as (block-) diagonal matrices with

$$
\begin{aligned}
& \boldsymbol{A}_{i}\left(\tilde{\boldsymbol{z}}_{i}\right)=\operatorname{diag}\left[\alpha_{i}\left(\tilde{z}_{i 1}\right), \alpha_{i}\left(\tilde{z}_{i 2}\right), \ldots, \alpha_{i}\left(\tilde{z}_{i T_{i}}\right)\right], \\
& \boldsymbol{B}_{i}\left(\tilde{\boldsymbol{z}}_{i}\right)=\left[\begin{array}{llll}
\boldsymbol{B}_{1 i}\left(\tilde{\boldsymbol{z}}_{i}\right) & \boldsymbol{B}_{2 i}\left(\tilde{\boldsymbol{z}}_{i}\right) & \ldots & \boldsymbol{B}_{m i}\left(\tilde{\boldsymbol{z}}_{i}\right)
\end{array}\right], \\
& \boldsymbol{B}_{\ell i}\left(\tilde{\boldsymbol{z}}_{i}\right)=\operatorname{diag}\left[\beta_{\ell i}\left(\tilde{z}_{i 1}\right), \beta_{\ell i}\left(\tilde{z}_{i 2}\right), \ldots, \beta_{\ell i}\left(\tilde{z}_{i T_{i}}\right)\right], \quad \ell=1,2, \ldots, m, \\
& \boldsymbol{\Psi}_{i}\left(\tilde{\boldsymbol{z}}_{i}\right)=\left[\begin{array}{llll}
\boldsymbol{\Psi}_{1 i}\left(\tilde{\boldsymbol{z}}_{i}\right) & \boldsymbol{\Psi}_{2 i}\left(\tilde{\boldsymbol{z}}_{i}\right) & \ldots & \boldsymbol{\Psi}_{n i}\left(\tilde{\boldsymbol{z}}_{i}\right)
\end{array}\right], \\
& \boldsymbol{\Psi}_{\ell i}\left(\tilde{\boldsymbol{z}}_{i}\right)=\operatorname{diag}\left[\psi_{\ell i}\left(\tilde{z}_{i 1}\right), \psi_{\ell i}\left(\tilde{z}_{i 2}\right), \ldots, \psi_{\ell i}\left(\tilde{z}_{i T_{i}}\right)\right], \quad \ell=1,2, \ldots, n,
\end{aligned}
$$

and

$$
\boldsymbol{\Theta}_{i}\left(\tilde{\boldsymbol{z}}_{i}\right)=-\boldsymbol{A}_{i}\left(\tilde{\boldsymbol{z}}_{i}\right)^{-1} \boldsymbol{B}_{i}\left(\tilde{\boldsymbol{z}}_{i}\right) .
$$

Note that

$$
\begin{aligned}
& \boldsymbol{\Theta}_{i}\left(\tilde{\boldsymbol{z}}_{i}\right)=\left[\begin{array}{llll}
\boldsymbol{\Theta}_{1 i}\left(\tilde{\boldsymbol{z}}_{i}\right) & \boldsymbol{\Theta}_{2 i}\left(\tilde{\boldsymbol{z}}_{i}\right) & \ldots & \boldsymbol{\Theta}_{m i}\left(\tilde{\boldsymbol{z}}_{i}\right)
\end{array}\right], \\
& \boldsymbol{\Theta}_{\ell i}\left(\tilde{\boldsymbol{z}}_{i}\right)=\operatorname{diag}\left[\theta_{\ell i}\left(\tilde{z}_{i 1}\right), \theta_{\ell i}\left(\tilde{z}_{i 2}\right), \ldots, \theta_{\ell i}\left(\tilde{z}_{i T_{i}}\right)\right], \quad \ell=1,2, \ldots, m,
\end{aligned}
$$

satisfying

$$
\theta_{\ell i}\left(\tilde{z}_{i t}\right)=-\alpha_{i}\left(\tilde{z}_{i t}\right)^{-1} \beta_{\ell i}\left(\tilde{z}_{i t}\right)
$$

Now, to represent the coefficients using polynomials in the conditioning state variable $\tilde{z}_{i t}$, define the matrix of polynomial elements up to order $\tau$ as

$$
\boldsymbol{\Pi}_{\tau}\left(\tilde{\boldsymbol{z}}_{i}\right)=\left[\begin{array}{llll}
c_{0}\left(\tilde{\boldsymbol{z}}_{i}\right) & c_{1}\left(\tilde{\boldsymbol{z}}_{i}\right) & \ldots & c_{\tau}\left(\tilde{\boldsymbol{z}}_{i}\right)
\end{array}\right]
$$

which has dimension $T_{i} \times(\tau+1)$, given that the columns of $\boldsymbol{\Pi}_{\tau}\left(\tilde{\boldsymbol{z}}_{i}\right)$ are constructed as

$$
c_{s}\left(\tilde{\boldsymbol{z}}_{i}\right)=\left[\begin{array}{llll}
c_{s}\left(\tilde{z}_{i 1}\right) & c_{s}\left(\tilde{z}_{i 2}\right) & \ldots & c_{s}\left(\tilde{z}_{i T}\right)
\end{array}\right]^{\prime}, \quad s=0,1, \ldots, \tau .
$$


The model coefficients are then specified as follows:

$$
\begin{aligned}
& \boldsymbol{\alpha}_{i}\left(\tilde{\boldsymbol{z}}_{i}\right)=\boldsymbol{\Pi}_{\tau}\left(\tilde{\boldsymbol{z}}_{i}\right) \boldsymbol{\gamma}_{i}^{(\alpha)} \\
& \gamma_{i}^{\alpha}=\left[\begin{array}{llll}
\gamma_{i 0}^{(\alpha)} & \gamma_{i 1}^{(\alpha)} & \ldots & \gamma_{i \tau}^{(\alpha)}
\end{array}\right]^{\prime} \\
& \boldsymbol{\beta}_{\ell i}\left(\tilde{\boldsymbol{z}}_{i}\right)=\boldsymbol{\Pi}_{\tau}\left(\tilde{\boldsymbol{z}}_{i}\right) \boldsymbol{\gamma}_{i}^{\left(\beta_{\ell i}\right)}, \quad \ell=1,2, \ldots, m, \\
& \gamma_{i}^{\left(\beta_{\ell i}\right)}=\left[\begin{array}{llll}
\gamma_{i 0}^{\left(\beta_{\ell i}\right)} & \gamma_{i 1}^{\left(\beta_{\ell i}\right)} & \ldots & \gamma_{i \tau}^{\left(\beta_{\ell i}\right)}
\end{array}\right]^{\prime}, \\
& \boldsymbol{\psi}_{\ell i}\left(\tilde{\boldsymbol{z}}_{i}\right)=\boldsymbol{\Pi}_{\tau}\left(\tilde{\boldsymbol{z}}_{i}\right) \boldsymbol{\gamma}_{i}^{\left(\psi_{\ell i}\right)}, \quad \ell=1,2, \ldots, n, \\
& \gamma_{i}^{\left(\psi_{\ell i}\right)}=\left[\begin{array}{llll}
\gamma_{i 0}^{\left(\psi_{\ell i}\right)} & \gamma_{i 1}^{\left(\psi_{\ell i}\right)} & \ldots & \gamma_{i \tau}^{\left(\psi_{\ell i}\right)}
\end{array}\right]^{\prime} .
\end{aligned}
$$

Inserting these polynomial specifications for the coefficients into the right-hand side terms of (A.1), we obtain

$$
\begin{aligned}
\boldsymbol{B}_{i}\left(\tilde{\boldsymbol{z}}_{i}\right) \tilde{\boldsymbol{x}}_{i} & =\sum_{\ell=1}^{m} \boldsymbol{B}_{\ell i}\left(\tilde{\boldsymbol{z}}_{i}\right) \boldsymbol{x}_{\ell i} \\
& =\sum_{\ell=1}^{m} \operatorname{diag}\left[\boldsymbol{\Pi}_{\tau}\left(\tilde{\boldsymbol{z}}_{i}\right) \boldsymbol{\gamma}_{i}^{\left(\beta_{\ell i}\right)}\right] \boldsymbol{x}_{\ell i} \\
& =\sum_{\ell=1}^{m} \operatorname{diag}\left(\boldsymbol{x}_{\ell i}\right) \boldsymbol{\Pi}_{\tau}\left(\tilde{\boldsymbol{z}}_{i}\right) \boldsymbol{\gamma}_{i}^{\left(\beta_{\ell i}\right)} \\
& =\boldsymbol{\mathcal { X }}_{i}\left(\tilde{\boldsymbol{z}}_{i}\right) \boldsymbol{\gamma}_{i}^{\left(\boldsymbol{\beta}_{i}\right)},
\end{aligned}
$$

where

$$
\begin{aligned}
\mathcal{X}_{i}\left(\tilde{\boldsymbol{z}}_{i}\right) & =\left[\begin{array}{llll}
\boldsymbol{\mathcal { X }}_{1 i}\left(\tilde{\boldsymbol{z}}_{i}\right) & \boldsymbol{\mathcal { X }}_{2 i}\left(\tilde{\boldsymbol{z}}_{i}\right) & \ldots & \boldsymbol{\mathcal { X }}_{m i}\left(\tilde{\boldsymbol{z}}_{i}\right)
\end{array}\right] \\
\boldsymbol{\mathcal { X }}_{\ell i}\left(\tilde{\boldsymbol{z}}_{i}\right) & =\operatorname{diag}\left(\boldsymbol{x}_{\ell i}\right) \boldsymbol{\Pi}_{\tau}\left(\tilde{\boldsymbol{z}}_{i}\right), \quad \ell=1,2, \ldots, m, \\
\boldsymbol{\gamma}_{i}^{\left(\boldsymbol{\beta}_{i}\right)} & =\left[\begin{array}{llll}
\boldsymbol{\gamma}_{i}^{\left(\beta_{1 i}\right)^{\prime}} & \boldsymbol{\gamma}_{i}^{\left(\beta_{2 i}\right)^{\prime}} & \ldots & \boldsymbol{\gamma}_{i}^{\left(\beta_{m i}\right)^{\prime}}
\end{array}\right]^{\prime} .
\end{aligned}
$$

In analogous fashion, it holds that

$$
\boldsymbol{A}_{i}\left(\tilde{\boldsymbol{z}}_{i}\right) \boldsymbol{y}_{i,-1}=\mathcal{Y}_{i,-1}\left(\tilde{\boldsymbol{z}}_{i}\right) \boldsymbol{\gamma}_{i}^{\left(\alpha_{i}\right)}
$$

where

$$
\mathcal{Y}_{i,-1}\left(\tilde{\boldsymbol{z}}_{i}\right)=\operatorname{diag}\left(\boldsymbol{y}_{i,-1}\right) \boldsymbol{\Pi}_{\tau}\left(\tilde{\boldsymbol{z}}_{i}\right),
$$

and

$$
\boldsymbol{\Psi}_{i}\left(\tilde{\boldsymbol{z}}_{i}\right) \tilde{\boldsymbol{h}}_{i}=\mathcal{H}_{i}\left(\tilde{\boldsymbol{z}}_{i}\right) \boldsymbol{\gamma}_{i}^{\left(\boldsymbol{\psi}_{i}\right)}
$$

where

$$
\mathcal{H}_{i}\left(\tilde{\boldsymbol{z}}_{i}\right)=\left[\begin{array}{lllll}
\mathcal{H}_{1 i}\left(\tilde{\boldsymbol{z}}_{i}\right) & \mathcal{H}_{2 i}\left(\tilde{\boldsymbol{z}}_{i}\right) & \ldots & \mathcal{H}_{n i}\left(\tilde{\boldsymbol{z}}_{i}\right)
\end{array}\right]
$$




$$
\begin{gathered}
\boldsymbol{\mathcal { H }}_{\ell i}\left(\tilde{\boldsymbol{z}}_{i}\right)=\operatorname{diag}\left(\boldsymbol{h}_{\ell i}\right) \boldsymbol{\Pi}_{\tau}\left(\tilde{\boldsymbol{z}}_{i}\right), \quad \ell=1,2, \ldots, n, \\
\boldsymbol{\gamma}_{i}^{\left(\boldsymbol{\psi}_{i}\right)}=\left[\begin{array}{llll}
\boldsymbol{\gamma}_{i}^{\left(\psi_{1 i}\right)^{\prime}} & \boldsymbol{\gamma}_{i}^{\left(\psi_{2 i}\right)^{\prime}} & \ldots & \boldsymbol{\gamma}_{i}^{\left(\psi_{n i}\right)^{\prime}}
\end{array}\right]^{\prime}
\end{gathered}
$$

Using Equations (A.8) to (A.10), Equation (A.1) becomes

$$
\Delta \boldsymbol{y}_{i}=\mathcal{Y}_{i,-1}\left(\tilde{\boldsymbol{z}}_{i}\right) \boldsymbol{\gamma}_{i}^{\left(\alpha_{i}\right)}+\boldsymbol{\mathcal { X }}_{i}\left(\tilde{\boldsymbol{z}}_{i}\right) \boldsymbol{\gamma}_{i}^{\left(\boldsymbol{\beta}_{i}\right)}+\boldsymbol{\mathcal { H }}_{i}\left(\tilde{\boldsymbol{z}}_{i}\right) \boldsymbol{\gamma}_{i}^{\left(\boldsymbol{\psi}_{i}\right)}+\boldsymbol{\varepsilon}_{i}
$$

Once the coefficients in (A.11) have been estimated using country-specific least squares, we can use these in the second step to obtain the conditionally homogeneous long-run coefficients through pooled least-squares estimation of

$$
\boldsymbol{v}_{i}=-\Theta\left(\tilde{\boldsymbol{z}}_{i}\right) \tilde{\boldsymbol{x}}_{i}+\boldsymbol{\epsilon}_{i}
$$

where

$$
\begin{gathered}
\boldsymbol{v}_{i}=\hat{\boldsymbol{A}}_{i}\left(\tilde{\boldsymbol{z}}_{i}\right)^{-1}\left[\Delta \boldsymbol{y}_{i}-\boldsymbol{\mathcal { H }}_{i}\left(\tilde{\boldsymbol{z}}_{i}\right) \hat{\gamma}_{i}^{\left(\boldsymbol{\psi}_{i}\right)}\right]-\boldsymbol{y}_{i,-1}, \\
\boldsymbol{\epsilon}_{i}=\hat{\boldsymbol{A}}_{i}\left(\tilde{\boldsymbol{z}}_{i}\right)^{-1} \boldsymbol{\varepsilon}_{i},
\end{gathered}
$$

and

$$
V\left(\boldsymbol{\epsilon}_{i}\right)=\hat{\boldsymbol{A}}_{i}\left(\tilde{\boldsymbol{z}}_{i}\right)^{-2} \hat{\sigma}_{i}^{2}
$$

Using a polynomial specification for the long-run coefficients, namely

$$
\begin{gathered}
\boldsymbol{\theta}_{\ell i}\left(\tilde{\boldsymbol{z}}_{i}\right)=\boldsymbol{\Pi}_{\tau}\left(\tilde{\boldsymbol{z}}_{i}\right) \boldsymbol{\gamma}_{i}^{\left(\theta_{\ell}\right)}, \quad \ell=1,2, \ldots, m, \\
\boldsymbol{\gamma}_{i}^{\left(\theta_{\ell}\right)}=\left[\begin{array}{llll}
\gamma_{i 0}^{\left(\theta_{\ell}\right)} & \gamma_{i, 1}^{\left(\theta_{\ell}\right)} & \ldots & \gamma_{i \tau}^{\left(\theta_{\ell}\right)}
\end{array}\right]^{\prime}
\end{gathered}
$$

we have

$$
\Theta\left(\tilde{\boldsymbol{z}}_{i}\right) \tilde{\boldsymbol{x}}_{i,-1}=\boldsymbol{\mathcal { X }}_{i}\left(\tilde{\boldsymbol{z}}_{i}\right) \gamma^{(\boldsymbol{\theta})}
$$

with

$$
\gamma^{(\theta)}=\left[\begin{array}{llll}
\gamma^{\left(\theta_{1}\right)^{\prime}} & \gamma^{\left(\theta_{2}\right)^{\prime}} & \ldots & \gamma^{\left(\theta_{m}\right)^{\prime}}
\end{array}\right]^{\prime}
$$

such that (A.12) finally becomes

$$
\boldsymbol{v}_{i}=-\mathcal{X}_{i}\left(\tilde{z}_{i}\right) \gamma^{(\boldsymbol{\theta})}+\boldsymbol{\epsilon}_{i}
$$

\section{B Computation of Smoothed Mean Group Estimates and Standard Errors for Speed of Adjustment Co- efficients}

Under the CPMG approach, we estimate $N$ separate functional forms for the speed of adjustment coefficients, such that

$$
\hat{\alpha}_{i t}^{(j)}=\hat{\alpha}_{j}\left(\tilde{z}_{i t}\right), \quad i=1,2, \ldots, N, t=1,2, \ldots, T_{i}, j=1,2, \ldots, N
$$


represents the estimate of the speed of adjustment evaluated at observation $(i, t)$ using the functional form estimated for country $j$. Similar to the MG approach we now want to obtain an estimate of the mean relationship in the panel between the speed of adjustment coefficient and the conditioning state variable $\tilde{z}_{i t}$ by averaging across country-specific estimates of this relationship. The country-specific functional forms are based on Chebyshev polynomials up to order $r$, with polynomial terms $c_{s}\left(\tilde{z}_{i t}\right)$ and parameters $\gamma_{j s}^{\left(\alpha_{j}\right)}, s=0,1, \ldots, r$. The mean coefficient at the point $\tilde{z}_{i t}$ should therefore be an average of the coefficients implied by each polynomial. However, the polynomial function for each country's speed of adjustment coefficient is estimated on the basis of the observations for that country only and therefore might only be valid in a limited range of values for $\tilde{z}_{i t}$. Extrapolating this function to values that are far from this range might lead to large outliers which can distort the panel MG coefficient.

We therefore compute a weighted average of the heterogeneous coefficients $\hat{\alpha}_{j}\left(\tilde{z}_{i t}\right)$, where the weights decrease with the distance of $\tilde{z}_{i t}$ from the mean for country $j, \bar{z}_{j}$. The distance may be incorporated using a kernel specification. In particular, let $\hat{\gamma}_{j}^{\left(\alpha_{j}\right)}$ be the $r+1$ vector of estimated polynomial coefficients for country $j$. Then

$$
\hat{\alpha}_{i t}^{(j)}=\hat{\gamma}_{j}^{\left(\alpha_{j}\right)^{\prime}} \boldsymbol{\pi}_{r}\left(\tilde{z}_{i t}\right)
$$

where $\boldsymbol{\pi}_{r}\left(\tilde{z}_{i t}\right)=\left[c_{0}\left(\tilde{z}_{i t}\right), c_{1}\left(\tilde{z}_{i t}\right), \ldots, c_{r}\left(\tilde{z}_{i t}\right)\right]^{\prime}$. We now obtain the weights from the kernel specification

$$
\kappa_{i t}^{(j)}=\mathcal{K}\left(\frac{\tilde{z}_{i t}-\bar{z}_{j}}{h}\right)
$$

where $\mathcal{K}(\cdot)$ denotes the Gaussian kernel and $h$ the bandwidth, computed following Pagan and Ullah (1999, p. 26) as $h=1.06 \sigma_{\tilde{z}}\left(\sum_{i=1}^{N} T_{i}\right)^{-1 / 5}$, with $\sigma_{\tilde{z}}$ representing the overall standard deviation of $\tilde{z}_{i t}$. To ensure a proper definition of the weighted average, we standardize the weights using

$$
w_{i t}^{(j)}=\frac{\kappa_{i t}^{(j)}}{\sum_{k=1}^{N} \kappa_{i t}^{k}} .
$$

We finally are in a position to construct a smoothed mean group estimator (SMG) of the speed of adjustment coefficient from

$$
\hat{\alpha}_{i t}^{S M G}=\sum_{j=1}^{N} \hat{\alpha}_{i t}^{(j)} w_{i t}^{(j)}
$$

and the corresponding standard error from

$$
\hat{\sigma}_{\alpha, i t}^{S M G}=\sqrt{\frac{1}{N-1} \sum_{j=1}^{N}\left(\hat{\alpha}_{i t}^{(j)}-\hat{\alpha}_{i t}^{S M G}\right)^{2} w_{i t}^{(j)}} .
$$




\section{Kernel Specification}

Let us define $\kappa^{(h)}\left(\tilde{z}_{i t}-\tilde{z}_{j s}\right)=\mathcal{K}\left(\frac{\tilde{z}_{i t}-\tilde{z}_{j s}}{h}\right)$, where $\mathcal{K}(\cdot)$ denotes a standard kernel function such as the Gaussian kernel and $h$ an appropriate choice of bandwidth. Then for a given combination of cross-sectional reference point $j$ and time-series reference point $s$ the kernel matrix for country $i$ looks as follows:

$$
\boldsymbol{K}_{i}\left(\tilde{z}_{j s}\right)=\left(\begin{array}{cccc}
\kappa\left(\tilde{z}_{i 1}-\tilde{z}_{j s}\right) & & & 0 \\
& \kappa\left(\tilde{z}_{i 2}-\tilde{z}_{j s}\right) & & \\
& & \ddots & \\
0 & & \kappa\left(\tilde{z}_{i T_{i}}-\tilde{z}_{j s}\right)
\end{array}\right)_{T_{i} \times T_{i}}
$$

where for notational convenience the superscript $(h)$ has been dropped, as in Section 3.3. The full kernel matrix for all $t=1,2, \ldots, T_{i}, i=1,2, \ldots, N$, is constructed as

$$
\boldsymbol{K}\left(\tilde{z}_{j s}\right)=\left(\begin{array}{cccc}
\boldsymbol{K}_{1}\left(\tilde{z}_{j s}\right) & & & \mathbf{0} \\
& \boldsymbol{K}_{2}\left(\tilde{z}_{j s}\right) & & \\
& & \ddots & \\
0 & & & \boldsymbol{K}_{N}\left(\tilde{z}_{j s}\right)
\end{array}\right)_{\left(\sum_{i=1}^{N} T_{i}\right) \times\left(\sum_{i=1}^{N} T_{i}\right)}
$$

It is clear that the estimation results will be affected by the choice of both the kernel density function, $\mathcal{K}(\cdot)$, and the bandwidth parameter, $h$. Nevertheless, the specific kernel function is not crucial for the estimation results as for kernels belonging to the same class, the bandwidth parameter can be adjusted using "canonical kernels" such that the estimated functions are largely equivalent. ${ }^{22}$ Employing the Gaussian kernel, we follow Pagan and Ullah (1999, p. 26) and choose the bandwidth parameter as

$$
h=1.06 \sigma_{\tilde{z}}\left(\sum_{i=1}^{N} T_{i}\right)^{-1 / 5},
$$

where $\sigma_{\tilde{z}}$ is the standard deviation of the conditioning variable $\tilde{z}_{i t}$ across time and cross sections.

\section{Testing for the Existence of a Long-Run Relation- ship}

To compute the MG, PMG, CPMG and SKMG estimators, we need to be assured that a long-run relation between the dependent variable, $y$, and the regressors, $\boldsymbol{x}$, in the panel

\footnotetext{
${ }^{22}$ See, for example, Härdle (1990).
} 
ARDL model exists (unconditionally so for MG and PMG, and conditionally for CPMG and SKMG). Presuming that $y$ and $\boldsymbol{x}$ are integrated of order one, I(1), one may test whether they are cointegrated by considering a least squares regression of the form

$$
y_{i t}=\varpi_{i}+\boldsymbol{\theta}\left(\tilde{z}_{i t}\right)^{\prime} \boldsymbol{x}_{i t}+\xi_{i t}
$$

and examining whether the error term $\xi_{i t}$ in this regression is $\mathrm{I}(0)$ or $\mathrm{I}(1)$. If the null hypothesis is formulated as there being no cointegrating relation between $y_{i t}$ and $\boldsymbol{x}_{i t}$, then the error term $\xi_{i t}$ should be I(1). We employ the panel cointegration test proposed by Westerlund (2005) which implements this idea in a non-parametric format, not relying on specific assumptions regarding the data-generating processes for $y_{i t}$ and $\boldsymbol{x}_{i t}$. This makes the test applicable both when the conditioning function $\boldsymbol{\theta}(\tilde{z})$ collapses to a constant and when it exhibits variation across different values of $\tilde{z}$. All that is required is that $\boldsymbol{\theta}(\tilde{z})^{\prime} \boldsymbol{x}$ contains only $\mathrm{I}(0)$ and $\mathrm{I}(1)$ regressors.

The test also allows for cross-section dependence in the error term, $\xi_{i t}$, via common effects. To test the null hypothesis of no cointegration against the alternative hypothesis of cointegration for all countries, following Westerlund (2005) we compute the following panel variance ratio statistic:

$$
V R_{P}=\left(\sum_{i=1}^{N} \hat{\mathrm{u}}_{i}\right)^{-1} \sum_{i=1}^{N} \sum_{t=1}^{T} \hat{\mathrm{v}}_{i t}^{2},
$$

where $\hat{\mathrm{v}}_{i t}=\sum_{s=1}^{t} \hat{\xi}_{i s}$ and $\hat{\mathrm{u}}_{i}=\sum_{t=1}^{T} \hat{\xi}_{i t}^{2}$. This test statistic is distributed standard Normal under the null hypothesis of no cointegration after appropriate mean and variance corrections as reported by Westerlund (2005).

\section{E Valuation Adjustment}

In the compilation of our database we followed the principles for valuation adjustment described in Appendix A of Lane and Milesi-Ferretti (2001). In particular, equity assets (value of domestic holdings of foreign equity shares in U.S. Dollars) were adjusted by changes in the MSCI World Index, $m$, assuming that equity investment abroad is allocated according to the world portfolio that is approximated by this index. ${ }^{23}$ Decomposing the change in the stock into

$$
\Delta E Q A_{i t}=D E Q A_{i t}+\Delta V(E Q A)_{i t},
$$

where $D E Q A_{i t}$ refers to the flow of equity assets from country $i$ in period $t$, we computed the change in the value of the stock as

$$
\Delta V(E Q A)_{i t}=\left(\frac{m_{t}}{m_{t-1}}-1\right) E Q A_{i, t-1}+\left(\frac{m_{t}}{\sqrt{m_{t} m_{t-1}}}-1\right) D E Q A_{i t}
$$

\footnotetext{
${ }^{23}$ Note that for the United States, Japan and the UK we used an adjusted index that in each case excluded these countries from the definition of the rest of the world.
} 
taking into account that $m_{t}$ refers to end-of-period values, whereas flows are assumed to occur uniformly throughout the year and thus at an average value of $\sqrt{m_{t} m_{t-1}}$.

Equity liabilities (value of foreign holdings of domestic equity shares in U.S. Dollars) were adjusted by U.S. Dollar based changes in domestic (or regional) stock market indices, $m_{i}$, in the same vein as equity assets, with

$$
\Delta E Q L_{i t}=D E Q L_{i t}+\Delta V(E Q L)_{i t}
$$

$D E Q L_{i t}$ denoting the flow of equity liabilities to country $i$ in period $t$, and

$$
\Delta V(E Q L)_{i t}=\left(\frac{m_{i t}}{m_{i, t-1}}-1\right) E Q L_{i, t-1}+\left(\frac{m_{i t}}{\sqrt{m_{i t} m_{i, t-1}}}-1\right) D E Q L_{i t} .
$$

FDI assets on the basis of the book value method were adjusted for changes in the real (trade-weighted) U.S. Dollar exchange rate of country $i$ 's trade partners as follows:

$$
\Delta F D I A_{i t}=D F D I A_{i t}+\Delta V(F D I A)_{i t},
$$

where $D F D I A_{i t}$ denotes the flow of FDI assets from country $i$ in period $t$ and $\Delta V(F D I A)_{i t}$ the change in the value of country $i$ 's FDI asset stock from the end of period $t-1$ to the end of period $t$, with

$$
\begin{aligned}
& \Delta V(F D I A)_{i t}=\left(\frac{\tilde{q}_{i t}}{\tilde{q}_{i, t-1}}-1\right) F D I A_{i, t-1}, \\
& \tilde{q}_{i t}=\exp \left\{\sum_{j=1}^{N} \ln \left(\frac{C P I_{j t}}{C P I_{U S, t} \cdot s_{j t}}\right) \cdot w_{i j t}\right\},
\end{aligned}
$$

$C P I$ denoting the consumer price index and $s_{j t}$ country $j$ 's nominal bilateral exchange rate with the U.S. Dollar in units of domestic currency per one U.S. Dollar. The weight $w_{i j t}$ is calculated as country $i$ 's trade (the sum of exports, EXP, and imports, $I M P$ ) with country $j$ relative to country $i$ 's total trade during that year, namely

$$
w_{i j t}=\frac{E X P_{i j t}+I M P_{i j t}}{\sum_{k=1}^{N} E X P_{i k t}+I M P_{i k t}} .
$$

We thus assume that the foreign direct investment flows from a country are in line with its trade pattern, and that changes in the foreign direct investment position that country $i$ holds in country $j$ are due to changes in the relative price of consumption goods between country $j$ and the United States as well as changes in the value of country $j$ 's currency relative to the U.S. Dollar.

FDI liabilities were adjusted using analogous formulae, namely:

$$
\Delta F D I L_{i t}=D F D I L_{i, t-1}+\Delta V(F D I L)_{i t}
$$


where $D F D I L_{i t}$ denotes the flow of FDI liabilities to country $i$ in period $t$ and the change in the value of country $i$ 's FDI liability stock is defined as

$$
\Delta V(F D I L)_{i t}=\left(\frac{q_{i t}}{q_{i, t-1}}-1\right) F D I L_{i, t-1}
$$

with

$$
q_{i t}=\frac{C P I_{i t}}{C P I_{U S, t} \cdot s_{i t}}
$$

Finally, we inferred changes to the stock of international reserves excluding gold holdings $\left(R E S^{*}\right)$ from the difference between the change in official reserves $(\triangle R E S)$ according to IIP and recorded reserve flows $(D R E S)$ :

$$
\Delta V\left(R E S^{*}\right)_{i t}=\Delta R E S_{i t}-D R E S_{i t}
$$

Consequently, the net valuation change used for adjusting the cumulative flow measure for NFA (taking into account once more that changes in the value of external debt are already incorporated in the stock values reported in the GDF database) was constructed as

$$
\Delta N V_{i t}=\Delta V(F D I A)_{i t}-\Delta V(F D I L)_{i t}+\Delta V(E Q A)_{i t}-\Delta V(E Q L)_{i t}+\Delta V\left(R E S^{*}\right)_{i t}
$$




\section{References}

Baum, C.F., J.T. Barkoulas and M. Caglayan (2001): Nonlinear Adjustment to Purchasing Power Parity in the Post-Bretton Woods Era, Journal of International Money and Finance, 20(3), 379-399.

Binder, M., M.H. Pesaran and S. Sharma (2004): Common Features in Exchange Rate Dynamics Using Dynamic Heterogeneous Panel Data Models, Working Paper, Goethe University Frankfurt.

Breitung, J. (2005): A Parametric Approach to the Estimation of Cointegration Vectors in Panel Data, Econometric Reviews, 24(2), 151-173.

Cavallo, M. and F. Ghironi (2002): Net Foreign Assets and the Exchange Rate: Redux Revived, Journal of Monetary Economics, 49(5), 1057-1097.

Cheung, Y.W., M.D. Chinn and A.G. Pascual (2005): Empirical Exchange Rate Models of the Nineties: Are Any Fit to Survive?, Journal of International Money and Finance, $24(7), 1150-1175$.

Cheung, Y.W. and K.S. Lai (2000): On Cross-Country Differences in the Persistence of Real Exchange Rates, Journal of International Economics, 50(2), 375-397.

Engel, C., N.C. Mark and K. West (2007): Exchange Rate Models Are Not as Bad as You Think, Working Paper, University of Wisconsin.

Fan, J. and W. Zhang (1999): Statistical Estimation in Varying Coefficient Models, Annals of Statistics, 27(5), 1491-1518.

Hall, R.E. and C.I. Jones (1999): Why Do Some Countries Produce So Much More Output Per Worker Than Others?, Quarterly Journal of Economics, 114(1), 83-116.

Härdle, W. (1990): Applied Nonparametric Regression, Cambridge, UK: Cambridge University Press.

Kumar, S. and A. Ullah (2000): Semiparametric Varying Parameter Panel Data Models: An Application to Estimation of Speed of Convergence, in: T. B. Fomby and R. Carter Hill (Eds.), Advances in Econometrics, Stamford, Conn.: JAI Press, Vol. 14, pp. 109-128.

Lane, P.R. and G.M. Milesi-Ferretti (2001): The External Wealth of Nations: Measures of Foreign Assets and Liabilities for Industrial and Developing Countries, Journal of International Economics, 55(2), 263-294. 
Lane, P.R. and G.M. Milesi-Ferretti (2004): The Transfer Problem Revisited: Net Foreign Assets and Real Exchange Rates, Review of Economics and Statistics, 86(4), 841-857.

Lane, P.R. and G.M. Milesi-Ferretti (2005): Financial Globalization and Exchange Rates, Working Paper, International Monetary Fund.

Lane, P.R. and G.M. Milesi-Ferretti (2006): The External Wealth of Nations Mark II: Revised and Extended Estimates of Foreign Assets and Liabilities, 1970 - 2004, Working Paper, International Monetary Fund.

Levy-Yeyati, E. and F. Sturzenegger (2005): Classifying Exchange Rate Regimes: Deeds vs. Words, European Economic Review, 49(6), 1603-1635.

Mankiw, N.G., D. Romer and D.N. Weil (1992): A Contribution to the Empirics of Economic Growth, Quarterly Journal of Economics, 107(2), 407-437.

Obstfeld, M. and K. Rogoff (1995): Exchange Rate Dynamics Redux, Journal of Political Economy, 103(3), 624-660.

Offermanns, C.J. and M. Pramor (2006): The CFS International Capital Flow Database: A User's Guide, Working Paper, Goethe University Frankfurt.

Pagan, A. and A. Ullah (1999): Nonparametric Econometrics, Cambridge, UK: Cambridge University Press.

Pesaran, M.H. (2006): Estimation and Inference in Large Heterogeneous Panels with a Multifactor Error Structure, Econometrica, 74(4), 967-1012.

Pesaran, M.H. (2007): A Simple Panel Unit Root Test in the Presence of Cross Section Dependence, Journal of Applied Econometrics, 22(2), 265-312.

Pesaran, M.H. and Y. Shin (1999): An Autoregressive Distributed Lag Modelling Approach to Cointegration Analysis, in: S. Strom (Ed.), Econometrics and Economic Theory in the 20th Century: The Ragnar Frisch Centennial Symposium, Cambridge, UK: Cambridge University Press.

Pesaran, M.H., Y. Shin and R.P. Smith (1999): Pooled Mean Group Estimation of Dynamic Heterogeneous Panels, Journal of the American Statistical Association, 94(446), 621-634.

Pesaran, M.H. and R. Smith (1995): Estimating Long-Run Relationships from Dynamic Heterogeneous Panels, Journal of Econometrics, 68(1), 79-113.

Rogoff, K. (1996): The Purchasing Power Parity Puzzle, Journal of Economic Literature, $34(2), 647-668$. 
Sinn, S. (1990): Net External Asset Positions of 145 Countries: Estimation and Interpretation, in: H. Siebert (Ed.), Kieler Studien, Tübingen: Mohr, Vol. 234.

Taylor, A.M. and M.P. Taylor (2004): The Purchasing Power Parity Debate, Journal of Economic Perspectives, 18(4), 135-158.

Taylor, M.P., D.A. Peel and L. Sarno (2001): Nonlinear Mean-Reversion in Real Exchange Rates: Toward a Solution to the Purchasing Power Parity Puzzles, International Economic Review, 42(4), 1015-1042.

Westerlund, J. (2005): New Simple Tests for Panel Cointegration, Econometric Reviews, $24(3), 297-316$. 


\section{CFS Working Paper Series:}

No.

Author(s)

$2007 / 22$

Howard Kunreuther

Alexander Muermann

2007/21 Wolfram J. Horneff

Raimond H. Maurer

Olivia S. Mitchell

Michael Z. Stamos

2007/20 Bea Canto

Roman Kräussl

2007/19 Maria Kasch-Haroutounian Erik Theissen

2007/18 Günter W. Beck

Volker Wieland

2007/17 Günter W. Beck

Volker Wieland

2007/16 Silvio Colarossi

Andrea Zaghini

2007/15 Annamaria Lusardi

Olivia S. Mitchell

2007/14 Jean Boivin

Marc P. Giannoni

Ilian Mihov

2007/13 Virgiliu Midrigan
Title

Self-Protection and Insurance with Interdependencies

Money in Motion: Dynamic Portfolio Choice in Retirement

Electronic Trading Systems and Intraday NonLinear Dynamics: An Examination of the FTSE 100 Cash and Futures Returns

Competition between Exchanges: Euronext versus Xetra

Money in Monetary Policy Design under Uncertainty: A Formal Characterization of ECBStyle Cross-Checking

Money in Monetary Policy Design under Uncertainty: The Two-Pillar Phillips Curve versus ECB-Style Cross-Checking

Gradualism, Transparency and Improved Operational Framework: A Look at the Overnight Volatility Transmission

Financial Literacy and Retirement Preparedness: Evidence and Implications for Financial Education Programs

Sticky Prices and Monetary Policy: Evidence from Disaggregated U.S. Data

Menu Costs, Multi-Product Firms, and Aggregate Fluctuations

Copies of working papers can be downloaded at http://www.ifk-cfs.de 